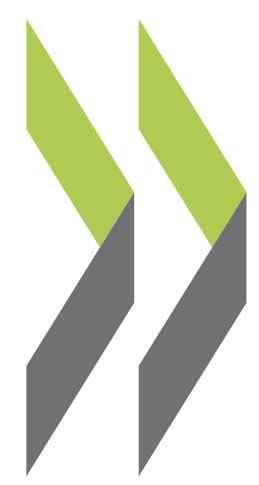

OECD Economics Department Working Papers No. 596

Explaining Differences in Hours Worked among OECD Countries: An empirical analysis

\title{
Orsetta Causa
}


Organisation de Coopération et de Développement Economiques

Organisation for Economic Co-operation and Development

10-Mar-2008

ECONOMICS DEPARTMENT

English - Or. English

ECONOMICS DEPARTMENT WORKING PAPERS No. 596

EXPLAINING DIFFERENCES IN HOURS WORKED AMONG OECD COUNTRIES: AN EMPIRICAL ANALYSIS

By Orsetta Causa

All Economics Department Working Papers are available through OECD's Internet website at http://www.oecd.org/eco

JT03242014 


\section{Explaining differences in hours worked among OECD countries: an empirical analysis}

This working paper investigates the policy determinants of hours worked among employed individuals in OECD countries, focussing on the impact of taxation, working-time regulations, and other labour and product market policies. It explores the factors underlying cross-country differences in hours worked - in line with previous aggregate approaches - while at the same time it looks more closely at labour force heterogeneity - in the vein of microeconomic labour supply models. The paper shows that policies and institutions have a different impact on working hours of men and women. Firstly, while high marginal taxes create a disincentive to work longer hours for women, their impact on hours worked by men is almost insignificant. Secondly, working-time regulations have a significant impact on hours worked by men, and this impact differs across education categories. Thirdly, other labour and product market policies, in particular stringent employment protection of workers on regular contracts and competition-restraining product market policies, have a negative impact on hours worked by men, over and beyond their impact on employment levels.

JEL codes: J22; J58; H31

Keywords: working hours; labour supply; taxation; working time regulations; labour market policy.

\section{Expliquer les différences d'heures travaillées dans les pays de l'OCDE: une analyse empirique}

Résumé: Cet article analyse les déterminants politiques des heures travaillées par la population employée dans les pays de l'OCDE. Ce travail porte sur l'impact des taxes, des réglementations du temps de travail, et des politiques du marché du travail et du marché des produits sur la marge intensive de l'utilisation du travail. Il s'interroge sur les facteurs sous-jacents les différences d'heures travaillées — en ligne avec les approches agrégées - mais analyse également l'hétérogénéité de la force de travail-dans la veine des analyses microéconomiques de l'offre de travail. Cet article montre que les politiques et les institutions ont un impact sur les heures travaillées par différentes sous-populations composant la force de travail. Pour résumer, tandis que les heures travaillées par les femmes sont sensibles à la fiscalité du travail, les heures travaillées par les hommes répondent davantage aux réglementations sur la durée du temps de travail ainsi qu'aux politiques du marché du travail et du marché des produits. Premièrement, alors qu'un niveau élevé de taxation marginale implique une désincitation à augmenter le nombre d'heures travaillées chez les femmes, l'impact de la fiscalité sur les heures travaillées par les hommes est nul. Deuxièmement, la réglementation sur la durée du temps de travail a un impact significatif sur les heures travaillées par les hommes, et cet impact varie en fonction du niveau d'éducation. Troisièmement, d'autres politiques structurelles, et en particulier la rigueur de la protection de l'emploi sur les contrats permanents, ainsi qu'une réglementation anti compétitive du marché des produits, ont un impact négatif sur les heures travaillées par les hommes, par-delà leur impact sur leur niveau d'emploi.

Classifications JEL: J22 ; J58 ; H31

Mots-clé: heures travaillées ; offre de travail ; taxation ; réglementation du temps de travail ; politique du marché du travail.

Copyright OECD, 2007

Application for permission to reproduce or translate all, or part of, this material should be made to: Head of Publications Service, OECD, 2 rue André Pascal, 75775 Paris Cedex 16, France. 


\section{TABLE OF CONTENTS}

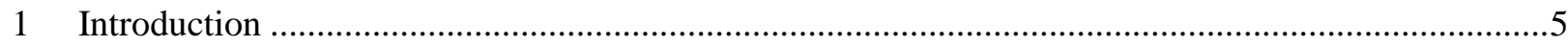

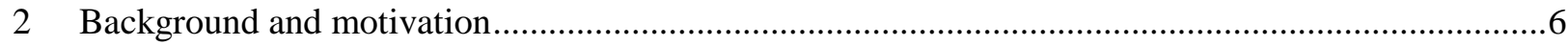

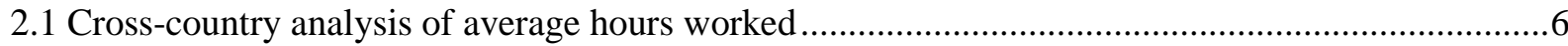

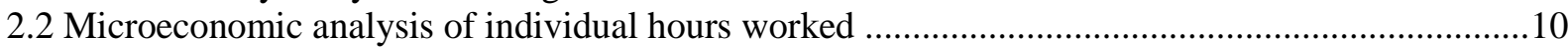

2.2.1 The standard individual labour supply model.................................................................... 10

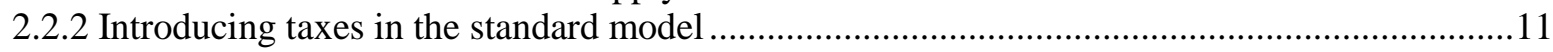

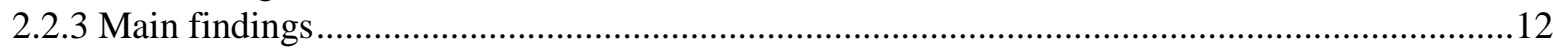

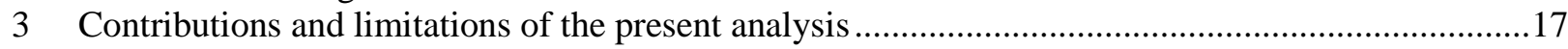

3.1 The issue: analyse the determinants of the intensive margin of labour supply ...............................17

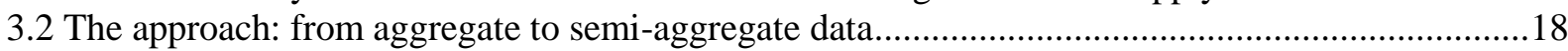

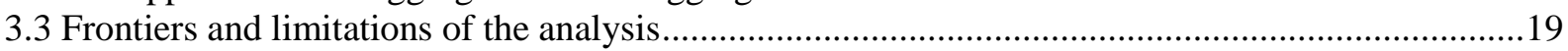

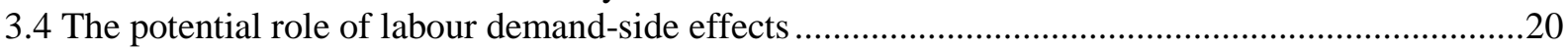

4 Cross-country analysis: who drives the aggregate relationship? A reassessment..............................22

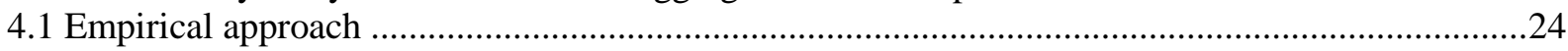

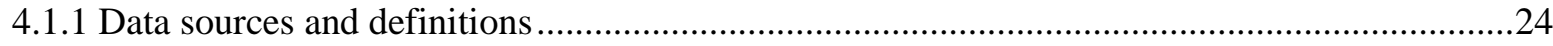

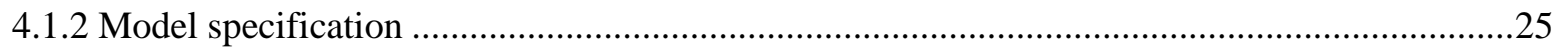

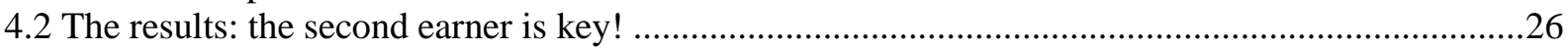

5 Semi-aggregate analysis: an assessment of the role of taxes and other policies...............................28

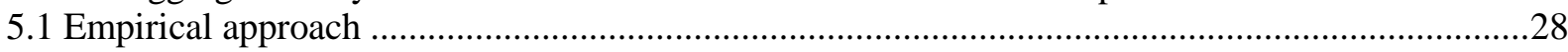

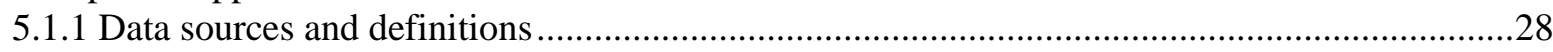

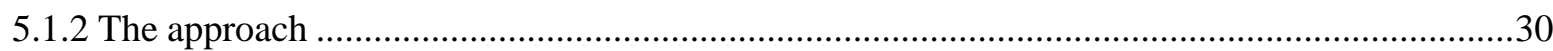

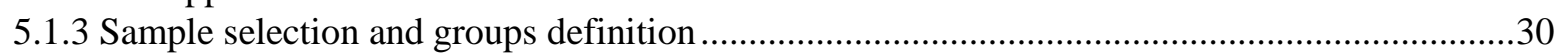

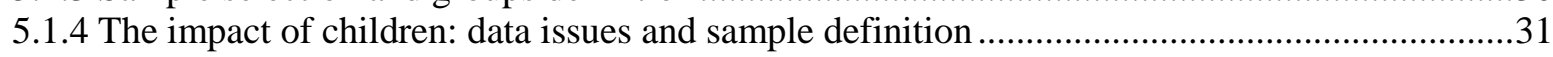

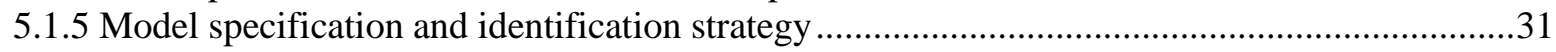

5.2 The results: the differential impact of taxes and policies across genders ..................................33

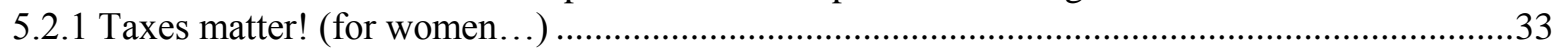

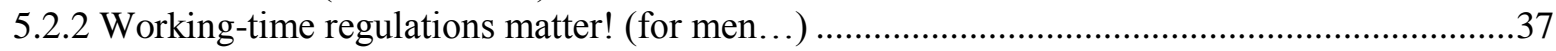

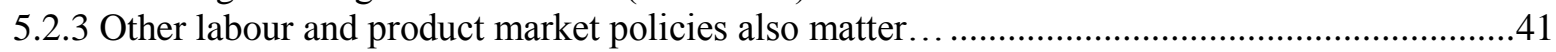

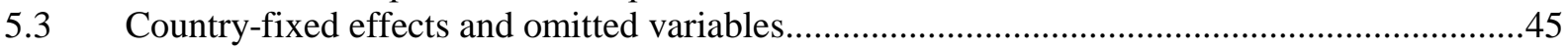

5.3.1 The measure of our ignorance: the importance of country-fixed effects ...................................45

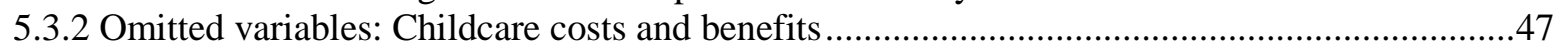

5.3.3 Omitted variables: Income-tested or hours-dependent benefits...............................................50

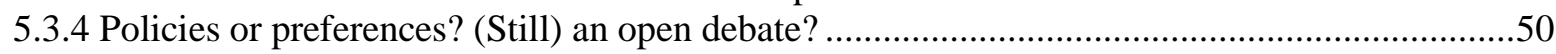

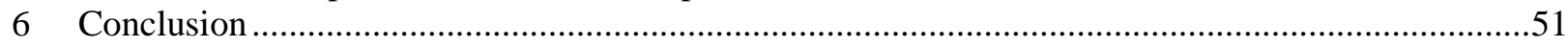

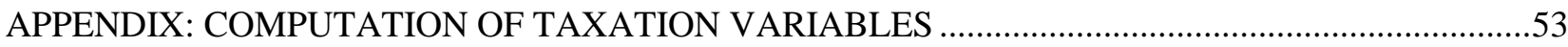

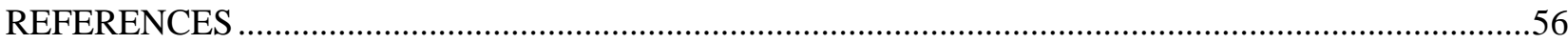

\section{Tables}

1. Cross-country literature on average hours worked: main findings

2. Microeconomic literature: Empirical labour supply models, women

3. Microeconomic literature: Empirical labour supply models, men

4. Econometric estimates - Aggregate results

5. Econometric estimates - Taxes

6. Econometric estimates - Working time regulations

7. Econometric estimates - Product and labour market policies 


\section{Figures}

1. Annual average hours worked per person on employment and aggregate measures of taxation

2. Weekly hours worked among employees and average marginal tax wedges - Women

3. Weekly hours worked among employees and working time regulations

4. Hours impact of country fixed effects, men and women

5. Childcare costs and country fixed effects

6. Country fixed effects in the regression for women and marginal effective tax rates for part time employees 
ECO/WKP(2008)4

\title{
EXPLAINING DIFFERENCES IN HOURS WORKED AMONG OECD COUNTRIES: AN EMPIRICAL ANALYSIS
}

\author{
By Orsetta Causa ${ }^{1}$
}

\section{1}

Introduction

1. "Are Europeans lazy or Americans crazy?" This was the provocative question debated at a recent conference of a number of influential economists from the two sides of the Atlantic (Fondazione Rodolfo de Benedetti, 2006). Indeed, gaps in incomes per capita between the United States and European countries are mostly accounted for by low labour utilisation, measured by hours worked per person (OECD, 2007). However, this reflects two distinct features of labour utilisation: employment and participation - or the extensive margin - and hours worked by employed individuals - or the intensive margin. While there is abundant literature on the policy determinants of participation and employment rates, ${ }^{2}$ the policy determinants of hours worked among the employed have been less studied.

2. The purpose of this paper is to fill in this gap by investigating the policy determinants of the intensive margin of labour utilisation among OECD countries. This work focuses on the impact of taxation, working-time regulations, and other labour and product market policies on working hours of employed individuals. It attempts to reconcile individual and cross-country empirical approaches to labour supply. Indeed, the microeconomic literature has extensively documented the heterogeneous labour supply behaviour of specific groups of the labour force, depending on gender, socioeconomic status and education level. The analysis undertaken here investigates the factors underlying cross-country differences in hours worked - in line with previous aggregate approaches - while at the same time looking more closely at labour force heterogeneity — in the vein of microeconomic labour supply models.

3. The paper shows that policies and institutions have an impact on working hours of different groups in the labour force. Firstly, while high marginal taxes create a disincentive to work longer hours for women, their impact on hours worked by men is almost insignificant. Secondly, working-time regulations have a significant impact on hours worked by men, and this impact differs across education categories. Thirdly, other labour and product market policies, in particular stringent employment protection of workers on regular contracts and anticompetitive product market policies have a negative impact on hours worked by men, over and beyond their impact on employment levels. Despite the finding of the importance of policies and institutions for understanding differences in hours worked among OECD countries, the empirical analysis shows that an important proportion of the observed differences remains unexplained and pertains to country-specific features. Understanding the nature of these specificities, and, in particular, the role of societal preferences, remains a topic for future research.

4. The paper is organised as follows. The first section sets up the theoretical and empirical contexts on which the analysis builds by reviewing the relevant cross-country and microeconomic literature. Against this background, the following section presents the contributions and limitations of the present approach. The fourth section provides a brief cross-country empirical analysis of the impact of taxes on

1. The author is especially grateful to Catherine Chapuis for outstanding research assistance, to Sven Blondal, Jean-Marc Burniaux, Jorgen Elmeskov, Jean-Luc Schneider, Caroline Abettan, Véronique Henriksson, Herwig Immervoll, John Martin, Christian Gianella, Stefano Scarpetta and Flavio Padrini for their help and comments, as well as several OECD colleagues for comments.

2. See, inter alia, Bassanini and Duval, 2006, Nunziata, 2005, Calmfors and Driffil, 1988, Nickell and Layard, 1999. 
hours worked. The fifth section is devoted to the core analysis of this paper, namely that of the impact of taxes, labour market regulations, and other labour and product market policies, on differences in hours worked among OECD countries for specific subgroups of employed individuals. The sixth section analyses the role of the country-fixed effects and their possible interpretation in the context of the empirical model. The last section concludes.

\section{$2 \quad$ Background and motivation}

\subsection{Cross-country analysis of average hours worked}

5. A voluminous recent literature explores the impact of tax policies and labour market institutions on average hours worked in OECD countries. The main result from this analysis is that differences in tax rates go a long way in explaining differences in average hours worked in OECD countries. A large number of these contributions have employed time-series cross-country econometric methodology, while others, less numerous but nevertheless influential, have adopted a calibration approach. Most of these papers have focussed on hours worked per capita or per working-age population, thus conflating the intensive and the extensive margins of the labour supply. ${ }^{4}$

6. In a recent article, Prescott (2004) argues that "virtually all of the large differences between U.S. labour supply and those in Germany and France are due to differences in tax systems". This result is obtained by calibrating a general equilibrium model of investment and labour supply on the populations of major advanced industrial countries over the periods 1970-1974 and 1993-1996. Given the differences in hours worked and tax rates among rich countries, and in particular given the differences between the United States and European countries, the policy implications are clear: by modifying tax systems, and for instance by decreasing marginal tax rates, Europe would go a long way in increasing labour utilisation to United States levels.

7. In a similar vein, Ohanian et al. (2006) also use a calibrated model to assess the role of taxation in explaining cross-country trend changes in hours worked per person at working age. Their results suggest that taxes - on consumption and income - can account for much of the variation in hours worked, both over time and across countries.

8. Recent work by Rogerson (2005) supports Prescott's hypothesis by proposing an alternative interpretation. The author suggests that reconciling this thesis with the Scandinavian model of high taxes and high total hours worked (i.e. employment rates times average hours worked) requires analysing the composition of government spending. Rogerson (2006) then shows that it matters whether tax proceeds are returned to household as a lump-sum transfer, or wether the size of the transfer is affected by the amount of labour supplied. For example, if high taxes are used to subsidise child care for individuals who work, then the effect on hours worked will be less than under a lump-sum transfer case. The author shows that by

3. This section does not present an important strand of the literature that stresses the role of technological change in understanding the cross-country and time-series variation in hours worked. This literature, though relevant, is not reviewed here because it mostly focuses on the convergence process of European countries towards US standards in the period from the end of World War II towards the mid-1990s. There are two main reasons why this hypothesis is not explored in the present work: the first is that the technology approach is a long-run one, whereas the present work exploits recent cross-country data over a short period of time (1996-2005). The second related reason is the homogeneity of the countries used in this study over the sample period in terms of technological development. For a global long-run view on the respective role of taxes and technological change in accounting for the differences in hours worked across the past four decades, see Rogerson (2005).

4. Exceptions in this respect are Davis and Henrekson (2004), and Faggio and Nickell (2007). 
holding the tax rate constant, these compositional changes can have important quantitative effects. This argument, however, does not apply to the intensive margin of labour supply, but rather to the comparison of employment levels among continental and Northern European countries.

9. A number of empirical papers have supported the view that taxes play an important role in explaining differences in labour utilisation across countries. Davis and Henrekson (2004) find some evidence of a negative relationship between the average tax wedge (including consumption taxes) on hours worked both per adult person and per worker. This result is confirmed by Dew-Becker and Gordon (2006) by using hours worked per capita in a wider sample of OECD countries covering a longer time span. Faggio and Nickell (2007) use panel-data techniques and find a negative impact of the average tax wedge on hours worked per employed individual.

10. It is important to note, however, that the negative relationship between tax rates and hours worked per employed individual has been found to be much stronger, or statistically significant, when the regression specification omits country-fixed effects. This result is acknowledged in Davis and Henrekson (2004) and confirmed in Alesina et al. (2005). The omission of country-fixed effects creates well-known biases in cross-country estimates; importantly, country-specific omitted factors, correlated with both taxes and hours worked, could be driving the negative relationship. Some studies, such as Faggio and Nickell (2007), however, have established a negative significant relationship despite the presence of country-fixed effects, suggesting that the literature has not converged on any consensual result on the relationship between aggregate data on taxes and hours worked. ${ }^{5}$

11. Despite tax rates being found to be important in explaining working-time differences across OECD countries, alternative explanations are as numerous as they are diverse. While also finding mild support for the role of average tax rates, Alesina et al. (2005) uncover a negative relationship between union density and hours worked per working-age person. These authors argue that European labour market regulations, advocated by unions in declining industries, who actively supported work-sharing arrangements, explain the bulk of the differences in working hours between the United States and Europe. These policies, it is claimed, had a society-wide influence on leisure patterns because of a social multiplier where the returns to leisure increase as more people take longer vacations. This view is empirically supported by Hubermann and Minns (2005) using a longer time period and controlling for a number of other possible determinants.

12. Contrary to the above results, after controlling for income inequality, Bowles and Park (2005) provide some evidence of a positive relationship between union density and average hours worked per employed person. This result is also found in Faggio and Nickell (2007).

13. Cross-country time-series models of hours worked have also been estimated in the political science field by Burgoon and Baxandaal (2004) to study the influence of different political coalitions on working-time. Controlling for ruling governments (i.e. countries' political leadership), the study finds that union density has a positive impact on hours worked per employed worker. According to the study, one possible reason could be that unions have traditionally opposed the introduction of part-time contracts.

5. Note, however, that Faggio and Nickell (2007) use a more complex specification, where they introduce the marginal tax rates of the second earner at zero wage, the marginal tax rate of the second earner at $67 \%$ average production wage, the marginal tax rate of singles at average production wage, and the average tax wedge. The negative significant sign is found on the marginal tax rate on the second earner at $67 \%$ average production wage, as well as on the average tax wedge. The marginal tax rate on single individuals at average production wages exhibits a counter-intuitive positive sign; the marginal tax rate on non-working second earners displays a positive sign, that the authors interpret as an indication of the disincentives to work low hours when spouses face high marginal tax rates at zero hours, although it can be questioned whether this is the right indicator to measure fiscal disincentives to work part-time for second earners. 
However, this might have changed over time with rising employment (and union membership) of younger female cohorts which arguably have a higher preference for part-time work. Indeed, the study shows that the interaction of union density with female participation rates has a negative impact on average working hours.

14. Other views have been offered as potential explanations of the differences in working hours across developed countries. One argument stresses the importance of inequality. Bell and Freeman (2001) attribute the trend toward longer hours in the United States compared with Germany to rising inequality, arguing that those who work longer move up in the wage distribution at the workplace, and the gains for working hard are greater, the more unequal the wage distribution. In a recent novel contribution, Michelacci and Pijoan-Mas (2007) provide a model in which they specify the channel whereby wage inequality affects the return to working longer hours. A rise in the dispersion of job offers, which translates into higher within-skill wage inequality, raises the gains from obtaining better jobs and gives workers greater incentives to work longer hours; the effect is stronger as the labour market becomes tighter. The authors investigate how several other features of the labour market affect working-time decisions. Hence, a higher probability of becoming unemployed and a longer duration of unemployment reduce the rate of use of the stock of human capital accumulated through working time and thereby reduce the incentive to work longer hours. Michelacci and Pijoan-Mas quantify the role of labour market conditions in accounting for the diverging evolution of working time in the United States and the European Union by calibrating a labour market search model with frictions and find that differences in labour market conditions, in particular differences in within-skill wage inequality, can account for differences in the trend evolution of hours worked across the two sides of the Atlantic over the past 30 years.

15. It is, however, difficult to disentangle the separate influences on incentives, institutions, and policy, and to separate these factors from cultural and other fixed factors. Wage inequality may well be affected by local institutional environment, or the result of weaker unionisation rates. These forces may in turn be a product of deeper longstanding work ethic or the basic drive to emulate some reference group (Bowles and Park, 2004). Hence, while the idea that aggregate labour market conditions can have important effects on aggregate hours worked through their effect on hours per worker has several interesting implications, it raises the issue of identifying the policies and institutions that might actually shape the evolution of labour market conditions.

16. Other researchers have noted that Europeans took a good portion of their secular increase in productivity in reduced work intensity while Americans have instead taken it in more consumption. In a recent influential contribution, Blanchard (2004) argues that differences between American and Europeans hours worked are due to different preferences over consumption/leisure choices.

17. Table 1 reviews some recent empirical findings based on panel-data techniques. Three main points emerge from this brief overview: 
Table 1. Cross-country literature on average hours worked: main findings

\begin{tabular}{|c|c|c|c|c|c|c|}
\hline Study & Sam ple and estimation period & Dependent variable and source & Regressors - institutions & Regressors - others & Estimation method & $\begin{array}{l}\text { Estimated elasticity of hours } \\
\text { worked with respect to the tax } \\
\text { rate }^{1}\end{array}$ \\
\hline Alesina, Glaeser and Sacerdote (2005) & 18 OECD countries, 1960-1995. & $\begin{array}{l}\text { Annual work hours per person } 15- \\
64, \text { OECD. }\end{array}$ & $\begin{array}{l}\text { Average tax rate [-]; union density } \\
\text { rate } \\
{[-\cdots] \text {; EPL [--; only one regression]. }}\end{array}$ & & $\begin{array}{l}\text { OLS with and w ithout country year } \\
\text { fixed effects. }\end{array}$ & $\begin{array}{l}-0.50 \text { (Annual hours w orked per } \\
\text { person, without controlling for } \\
\text { country fixed effects);:-018 (") } \\
\text { (Annual hours w orked per person, } \\
\text { controlling for country fixed effects). }\end{array}$ \\
\hline Altonji and Oldham (2003) & $\begin{array}{l}9 \text { OECD countries, years: 1979, 1982, } \\
1984,1995 \text { and } 1999 .\end{array}$ & $\begin{array}{l}\text { Average annual hours actually } \\
\text { worked per person in employment, } \\
\text { OECD. }\end{array}$ & $\begin{array}{l}\text { Number of weeks of paid vacations } \\
\text { mandated by law [--]. }\end{array}$ & & $\begin{array}{l}\text { OLS w ith and w ithout country and } \\
\text { year fixed effects. }\end{array}$ & \\
\hline Bow les and Park (2005) & 10 OECD countries, 1963-1998. & $\begin{array}{l}\text { Ln(average annual work hours of } \\
\text { employed persons), OECD. }\end{array}$ & Union density rate $[+]$. & $\begin{array}{l}\text { Inequality indicator [+++]; Ln(real } \\
\text { w age) [-]; Ln(real GDP per capita) [-- } \\
\text { ]; unemployment rate [--]; female } \\
\text { share in employment [--]. }\end{array}$ & $\begin{array}{l}\text { OLS with country and year fixed } \\
\text { effects. }\end{array}$ & \\
\hline Burgoon and Baxandall (2004) & 18 OECD countries, not reported. & $\begin{array}{l}\text { A) annual hours per employee, } \\
\text { University of Groeningen; B B annual } \\
\text { hours per w orking age person, } \\
\text { University of Groeningen and OECD. }\end{array}$ & $\begin{array}{l}\text { Union density rate lagged } 5 \text { [A) +++; } \\
\text { B) }+++ \text {; ; w wage centralisation lagged } 5 \\
\text { [A) -; B) - -; union density X female } \\
\text { labour force participation lagged } 5 \text { [A] } \\
\text {--; only one regression. B) ---; only } \\
\text { one regression]; Ln(social } \\
\text { expenditure/GDP) lagged } 1 \text { (A) no. B). }\end{array}$ & $\begin{array}{l}\text { Hours worked lagged } 1 \text { [A) +++; only } \\
\text { one regression. B) +++; only one } \\
\text { regression]; share of Christian } \\
\text { ) Democratic government portfflolo } \\
\text { lagged } 5 \text { [A) no. B) ---]; share of } \\
\text { Liberal government portfolio lagged } 5 \\
\text { [A0 no; B) no]; share of Social } \\
\text { Democratic. }\end{array}$ & $\begin{array}{l}\text { FGLS (assuming heteroskedasticity } \\
\text { across countries) with and w ithout } \\
\text { country fixed effects. }\end{array}$ & $\begin{array}{l}-0.28(* * *) \text { (Annual hours w orked per } \\
\text { employee, without controlling for } \\
\text { country fixed effects); -0.05 (Annual } \\
\text { hours worked per employee, } \\
\text { controlling for country fixed effects, } \\
\text { lagged dependent variable, and the } \\
\text { interaction betw een union density } \\
\text { and femalal labour force } \\
\text { participation. }\end{array}$ \\
\hline Davis and Henrekson (2004) & $\begin{array}{l}13 \text { OECD countries, years: 1977, } \\
1983,1990 \text { and } 1995 .\end{array}$ & $\begin{array}{l}\text { A) annual work hours per adult, } \\
\text { OECD; B) annual work hours per } \\
\text { employed adult, OECD. }\end{array}$ & $\begin{array}{l}\text { Sum of average tax rates on income, } \\
\text { payrolls and consumption }[\mathrm{A})-; \mathrm{B})-] .\end{array}$ & & $\begin{array}{l}\text { OLS with and w ithout country and } \\
\text { year fixed effects. }\end{array}$ & $\begin{array}{l}-0.51\left({ }^{* *}\right) \text { (Annual hours w orked per } \\
\text { person, without controlling for } \\
\text { country fixed effects); ; } 0.12 \text { (Annual } \\
\text { hours worked per person, controlling } \\
\text { for country fixed effects). }\end{array}$ \\
\hline Dew - Becker and Gordon (2006) & 16 OECD countries, 1960-2004. & $\begin{array}{l}\text { Annual work hours per total } \\
\text { population, University of Groeningen. }\end{array}$ & Average tax w edge [---]. & & OLS with country fixed effects. & $\begin{array}{l}-0.40\left({ }^{(* *)} \text { (Annual hours worked per }\right. \\
\text { person, controlling for country fixed } \\
\text { effects). }\end{array}$ \\
\hline Faggio and Nickell (2007) & 17 OECD countries, 1981-1999. & $\begin{array}{l}\text { Average Annual Hours Worked per } \\
\text { person in employment, OECD Labour } \\
\text { Market Statistics. }\end{array}$ & $\begin{array}{l}\text { Union density [++], EPL [--], Marginal } \\
\text { tax rate, spouse (100APW, OAPW) } \\
{[++], \text { Marginal tax rate, spouse }} \\
\text { (100APW, 67APW) [--], Average Tax } \\
\text { wedge [--], Marginal rate single } \\
\text { (100APW) [+]. }\end{array}$ & $\begin{array}{l}\text { Business sector labour productivity } \\
{[+++] \text {, male unemployment [[+], }} \\
\operatorname{Ln}(50 \mathrm{p} / 10 \mathrm{p})[+++], \operatorname{Ln}(90 \mathrm{p} / 50 \mathrm{p})[+++] .\end{array}$ & $\begin{array}{l}\text { OLS with country and year fixed } \\
\text { effects. }\end{array}$ & 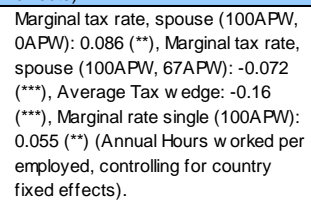 \\
\hline Huberman and Minns (2005) & 14 OECD, countries, $1950-2000$. & $\begin{array}{l}\text { Average annual w ork hours of total } \\
\text { w orkers, University of Groeningen. }\end{array}$ & Union density rate $[--\cdot]$. & 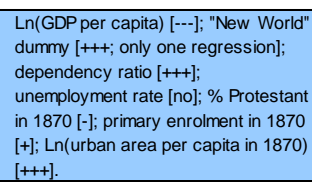 & & \\
\hline
\end{tabular}

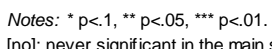

[no]: never significant in the main specifications or changing signs.

[+] or [-]: positively or negatively significant in a minority or half of the main specifications.

$[+++]$ or [---]: positively or negatively significant in all main specifications.

1. This estimate is approximated in cases where the presented results and data make it possible to compute it. It is defined as the estimated elasticity of hours w orked $w$ ith respect to the considered tax rate measure. When several taxation measures are used in a single estimation, the table presents the corresponding elasticities for each of them. This elasticity is the coefficient estimate obtained when regressing the log of hours worked on the tax rate. When the regression is defined in levels for the dependent variable, it can be approximated by dividing the slope coefficient by average hours w orked in the data used for estimation. 
- The most striking result from the cross-country literature stresses the negative relationship between average hours worked and average tax rates. However, cross-sectional time-series evidence on the relationship between hours worked and taxation, at the aggregate level, is weaker than the pure cross-sectional evidence, as stressed by Alesina et al. (2005). ${ }^{6}$

- This empirical finding at the aggregate level is not directly related to the theoretical framework underlying the relationship between taxes and market work. More specifically, microeconomic theory itself is ambiguous on the predicted impact of taxes on individual labour supply. Besides, the empirical and theoretical mechanisms through which aggregation over individual supply curves occur is ignored. Thus, somehow paradoxically, the taxes hypothesis remains unclear from a theoretical perspective.

- Alternative views on the causes of differences in hours worked do not result in any convincing story. The diversity of the proposed arguments seem to suggest, on the contrary, that countries' societal preferences, whether or not driven by a "multiplier" effect, go a long way towards explaining differences in rates of labour utilisation.

\subsection{Microeconomic analysis of individual hours worked}

18. The idea that taxes discourage individuals from market work is not an unambiguous prediction of economic theory. This section briefly reviews the theoretical framework underlying the relationship between taxes and hours worked at the individual level. A crucial theoretical distinction is made between labour supply choices at the extensive margin and choices at the intensive margin, and this has important empirical implications. Microeconomic literature has delivered important results in that respect; as mentioned before, though, cross-country models have rarely disentangled the impact of taxes on the two labour supply margins.

\subsubsection{The standard individual labour supply model}

19. In a static setting, individuals maximise a utility function over consumption and leisure. The resulting labour supply function depends on the marginal wage rate, $W$, representing the amount earned in real terms for an increase of hours work by one unit; on a measure of unearned income, $Y$, representing the amount of resources independent of an individual's work; and on a collection of other variables, $Z$, observed and unobserved, affecting labour supply:

20. For $\mathrm{H}$, defined as hours of market work, the above equation is the "structural" labour supply equation, holding tastes constant. Derivatives of this relationship are the neoclassical income and substitution effects of labour supply. A standard property of the labour supply function is manifested when examining the effect of a small increase in $\mathrm{W}$ on the supply of $\mathrm{H}: \frac{\delta \boldsymbol{h}}{\delta \boldsymbol{w}}$. The Slutzky equation decomposes this effect into a substitution effect, s, and an income effect, $\boldsymbol{h} \frac{\delta \boldsymbol{h}}{\delta \boldsymbol{y}}$ :

6. See the last column of Table 4.1, in which an attempt is made to compute, based on the reviewed articles, the implied elasticity of average hours worked with respect to the tax rate, in specifications including and excluding country-fixed effects, when the article makes this computation possible. This exercise has to be taken with care. Differences in data, samples, estimation techniques, and included regressors in the presented results make it extremely difficult to compare the implied elasticities across studies. The figures are only presented to convey a rough order of magnitude of the parameter (s) of interest. 


$$
\frac{\delta h}{\delta w}=s+h \frac{\delta h}{\delta y}
$$

21. The substitution effect, $\mathrm{s}$, measures the utility-constant (or income-compensated) effect of an increase in the wage on an individual's hours of work and the theory of constrained utility maximisation restricts $s$ to be positive: an increase in the wage rate raises the price of an hour not worked in the market, and at the same level of utility, this induces less consumption of non-market time and more time allocated to market work. At the same time, an increase in the wage rate augments the individual's wealth allowing him to consume more of those things that increase utility and to consume less of those things that generate disutility (such as hours of market work). This is the income effect of a wage increase on hours of market work and it is given by $h \frac{\delta h}{\delta y}$. This term is negative provided non-market time is a normal commodity. Consequently, the sign of the uncompensated effect of an increase in the individual's wage rate on hours of work (the left-hand side of the above equation) is indeterminate and depends on the relative magnitudes of the income and substitution effects.

\subsubsection{Introducing taxes in the standard model}

22. The literature applies two approaches for modelling the non-linearities induced by tax rates: piecewise-linear functions that reflect the brackets making up tax schedules; and smooth differentiable relations that summarise the tax rates implied by bracketed schedules. The two approaches share the same intuition, briefly described in this section.

23. The introduction of a non-linear tax schedule into a model of labour supply poses few analytical difficulties when the schedule generates a strictly convex constraint set with twice differentiable boundary. Utility maximisation in this case implies a simple characterisation of the hours-of-work choice. With $\tau$ denoting the smooth function that approximates the tax schedule, specify the "marginal wage rate" and "virtual" income as:

$$
\begin{aligned}
& \boldsymbol{w}=\boldsymbol{w}(\boldsymbol{h})=\left(1-\tau^{\prime}\right) \boldsymbol{W} \\
& \boldsymbol{y}=\boldsymbol{y}(\boldsymbol{h})=\boldsymbol{Y}+\boldsymbol{E}-\tau-\boldsymbol{W H}=\boldsymbol{Y}+\tau^{\prime} \boldsymbol{W h}-\tau=\boldsymbol{C}-\boldsymbol{w h}
\end{aligned}
$$

where $\mathrm{E}=\mathrm{Wh}$ is gross earnings and $\tau$ and $\tau^{\prime}$ (the derivative of the tax function with respect to income) are evaluated at income level $\mathrm{I}=\mathrm{I}(\mathrm{h})=\mathrm{Y}+\mathrm{Wh}$ (where $\mathrm{Y}$ is non-labour income), which directly depends on the value of $h$. Utility maximisation implies a solution for hours of work that obeys the implicit equation:

$$
\boldsymbol{h}=\boldsymbol{f}(\boldsymbol{w}(\boldsymbol{h}), \boldsymbol{y}(\boldsymbol{h}), v)
$$

With w(h) and $y(h)$ interpreted as "after-tax" measures, f continues to represent hours-of-work behaviour, even in the presence of complex non-linearities arising with tax systems. The objective of most labour supply analyses is to estimate the parameters of the function $\mathrm{f}$. The following equation considers a possible empirical counterpart to this labour supply model:

$$
\boldsymbol{h}=\boldsymbol{f}(\boldsymbol{w}(\boldsymbol{h}), \boldsymbol{y}(\boldsymbol{h}), v)=\kappa+\boldsymbol{Z} \chi+\alpha \boldsymbol{w}+\beta \boldsymbol{y}+v
$$

where $\mathrm{Z}$ is a vector of observed determinants of labour supply (age, family size, etc.), and $v$ is a structural disturbance capturing unobserved influences on the decision. The uncompensated wage effect is captured 
by the parameter $\alpha$, and, as explained above, has an ambiguous sign, while, provided leisure is not an inferior good, the income effect, captured by the parameter $\beta$, is expected to be positive.

\subsubsection{Main findings}

24. This section briefly summarises the most important findings emerging from the voluminous microeconomic literature on the values of labour supply elasticities in the presence of taxes. ${ }^{7}$ This presentation is limited to the standard static model of labour supply, therefore excluding intertemporal considerations. This choice is justified on several grounds, one of them being that the subsequent empirical analysis undertaken in this work is based on cross-country data available for a short period of time, at a level of disaggregation that does not allow individuals to be identified and therefore followed over their life-cycle.

25. Furthermore, the survey does not cover a recent strand of the literature that analyse the behaviour of high earners and conversely the value of earnings elasticities. The basic idea is that for many individuals, particularly the high earners and the self-employed - who have more discretion over their work intensity and for whom varied effort is likely to have greater short-run impact upon income - hours is not the exclusive margin of adjustment. By focusing on taxable income, it is possible to take account of some of these additional factors. Most of these studies make use of policy reforms for identifying earnings elasticities. Examples of this new approach are Moffit and Wilhem (2000), Saez (2003), and Eissa and Giertz (2006). The present study does not focus on high earners, and explicitly excludes the self-employed from the sample.

26. Acknowledging the above-mentioned limitations of this review, the findings developed here can be summarised in the following main points:

1. The labour participation decision is more responsive to wage and income variation than the decision about hours of work. ${ }^{8}$ This finding arises from the distinction between choices at the extensive margin and choices at the intensive margin (see Heckman, 1983, for a very illuminating representation of this distinction). The estimation of a structural labour supply model requires dealing with the problem of self-selection bias arising in sample of workers. Since Heckman $(1976,1979)$, researchers have been able to apply sample selection techniques in order to estimate structural models of labour supply. As is well-known, this bias has been mostly attributed to women's estimates, for whom participation rates are relatively low compared to men. Participation appears to be the key margin of adjustment, in particular for poor women, for which participation elasticities are very high. ${ }^{9}$

2. The estimated wage elasticity for women is positive, but there is a very wide range of dispersion among estimates (Blundell and MaCurdy, 1999, Cahuc and Zylberberg, 2003, Evers et al. 2006 ). Table 4.2 presents some of the findings of this large literature. According to a recent meta-analysis covering 239 elasticities (Evers et al. 2006), the range of variation for elasticities for women is from -0.19 to 2.79 , with a mean value of 0.41 and a median value of 0.28. Estimated elasticities of annual hours of work with respect to the wage rate are close to one. Estimates are lower when weekly hours are considered, because on an annual basis

7. For an extensive survey of the literature, see Handbook of Labour Economics, volume IIIA, chapter 27. For a meta-analysis, see Evers et al. (2006).

8. For empirical evidence on this, see Mroz (1987), Arrufat and Zabalza (1986) and Aaberge et al. (1999).

9. Analysis of the impact of government welfare programmes (e.g. the working families tax credit in the United Kingdom) supports this idea (Brewer and Browne, 2006). 
individuals have more margins of adjustment than on a weekly basis. Estimates of income elasticities depend on the various ways of measuring unearned income. Despite this variety, the range of estimates is not very wide, from -0.1 to -0.3 .

3. The estimated wage and income elasticities for men are very low and cluster around zero. Table 4.3 presents some of the relevant estimates. A variety of methods and datasets have been used and there is a clear consensus that the sensitivity of hours of work among men is extremely limited (see Pencavel, 1986).

4. Wage elasticities are declining with household income (see Aaberge et al. 1990, 1995, 1999). Thus, the aggregate impact of tax reform depends on the distribution of household earnings. Weak wage effects among middle-and high-income earners can counteract the impact of tax reform on poor households' labour supply.

27. Important extensions to the individual approach to labour supply include the development of a collective framework for the study of family labour supply and the introduction of household production (see Blundell and MaCurdy, 1999, for a discussion of this approach):

- Taking into account joint labour supply decisions allows explicitly to model the impact of an individual's earnings on the labour supply decision of another member of the family. ${ }^{10}$ Although the results from the empirical literature do not differ greatly from those delivered by the individual approach, ${ }^{11}$ taxation is likely to play a significant role in work sharing among first and second earners. Furthermore, the existence of strong cross-elasticities, in particular for married women, might induce a relatively modest impact of tax changes on aggregate labour supply. ${ }^{12}$

- Introducing household production is based on the idea that market work and home work are relatively substitutable. The important intuition of household production models is that households with lower opportunity costs of time in the market will engage in more home production for goods and services (e.g. meal preparation, laundry, child care) All else being equal, higher tax rates are associated with a higher proportion of time devoted to household production relative to market work (see Gronau, 1986, for the pioneering model, and Burda et al. 2006, for an in-depth analysis of its implications for understanding working-hours differences).

10. See Chiappori (1992) for the "income pooling" thesis.

11. See Attanasio and MaCurdy (1997), Kooreman and Kapteyn (1986), and Aaberge et al. (1999).

12. Aaberge et al. (1999) estimate a joint labour supply model with Italian data and find important cross elasticities of women's labour supply with respect to spouse's wage, in particular for the participation decision. This effect, combines with the rather inelastic labour supply among men and individuals with middle and high incomes, causes policy simulations to deliver a moderate aggregate impact on total labour supply. 


\begin{tabular}{|c|c|c|c|c|c|c|c|}
\hline Study & Country & Sample and estimation period & Variables used & Labour supply model & Estimation method & Uncompensated wage elasticity ${ }^{1}$ & Income elasticity \\
\hline Arellano and Meghir (1992) ${ }^{2}$ & United Kingdom & $\begin{array}{l}\text { British Family Expenditure Survey } 1983 \text { and } \\
\text { British Labour Force Survey } 1983 ; \text { sample } \\
\text { size } 11535 \text { employed; } 13200 \text { non } \\
\text { employed, aged 20.59. }\end{array}$ & $\begin{array}{l}\mathrm{d} \text { H: weekly hours; } W \text { : hourly earnings; } Y \text { : } \\
\text { consumplion based other income measure. }\end{array}$ & $\begin{array}{l}\text { Semi-log labour supply, convex. } \\
\text {. }\end{array}$ & Instrumental variables and selection. & 0.29 to 0.71 . & -0.13 to -0.40 \\
\hline Arrufat and Zabalza (1986) & United Kingdom & $\begin{array}{l}\text { British General Household Survey 1974; } \\
\text { sample size } 2002 \text { employed; } 1493 \text { non } \\
\text { employed aged 25-55. }\end{array}$ & $\begin{array}{l}\text { H: annual hours; W: gross hourly earnings; } \\
\text { Y: net w eekly unearned family income + } \\
\text { husband's earnings. }\end{array}$ & CES utilly based labour supply. & ML-convex, random preferences. & $\begin{array}{l}2.06 \text { (total): } 1.41 \text { (participation), } 0.62 \\
\text { (hours conditional on participation). }\end{array}$ & $\begin{array}{l}-0.20 \text { (total): :-0.14 (participation), }-0.06 \\
\text { (hours conditional on particication). }\end{array}$ \\
\hline Bargain (2005) & France & $\begin{array}{l}\text { 1994, French Household Budget Survey. } \\
\text { Married or cohabiting couples aged } 25-64 \text {. } \\
\text { Sample: } 3548 \text { couples. Participation rate for } \\
\text { women is } 77.2 \text { per cent. }\end{array}$ & $\begin{array}{l}\text { H: w eekly hours; } \mathrm{W} \text { : gross hourly } \\
\text { earnings; } \mathrm{Y} \text { : disposable income at each } \\
\text { r discrete choice of hours computed by } \\
\text { micro simulation of the French tax-benefit } \\
\text { system. }\end{array}$ & $\begin{array}{l}\text { Discrete choice models remaxing step by } \\
\text { step the restrictions usually imposed on } \\
\text { household preferences and rationality. }\end{array}$ & $\begin{array}{l}\text { Multinominal estimation of structural model, } \\
\text { unconstrained model, and general model } \\
\text { with price and income dependent } \\
\text { preferences, correcting for sample } \\
\text { selection into employment. }\end{array}$ & 0.30 (median). & not available. \\
\hline Blau and Kahn (2005) & United States & $\begin{array}{l}\text { March Current Population Survey data, } \\
\text { period: 1980-1990-2000. Sample: married } \\
\text { individuals aged 25-54 with a 25-54 } \\
\text { spouse present. Employment rate in 1970: } \\
0.67 \text {; in 1999: 0.78. }\end{array}$ & $\begin{array}{l}\text { H: annual hours, W: annual earnings/ } \\
\text { annual work hours for wage and salary } \\
\text { workers; Y: income from assets, including } \\
\text { interest, dividend, and rental income. } \\
\text { Spouse's wage is included. }\end{array}$ & $\begin{array}{l}\text { Linear supply model extended to include } \\
\text { spouse's wage. }\end{array}$ & $\begin{array}{l}\text { Instrumental variables estimation of annual } \\
\text { hours for those who worked with } \\
\text { selection correction. }\end{array}$ & 0.27 (conditional on participation, median). & -0.11 (conditional on participation, median). \\
\hline $\begin{array}{l}\text { Blomquist and Hansson-Brusewitz } \\
\text { (1990) }\end{array}$ & Sweden & $\begin{array}{l}\text { Sw edish Level of Living Survey 1981; } \\
\text { sample size } 795 \text { full sample, } 640 \text { employed; } \\
\text { all married, age 25-55. }\end{array}$ & $\begin{array}{l}\text { H: annual hours; W: directly observed, SS; } \\
\text {; Y: spouse's net income + family } \\
\text { allow ances + net capital income. }\end{array}$ & $\begin{array}{l}\text { Linear and quadratic labour supply, convex } \\
\text { and non convex. }\end{array}$ & $\begin{array}{l}\text { < Linear labour supply: ML-convex, ML- non } \\
\text { convex, random preferernces (on income } \\
\text { coefficient); Quadraicic labour supply: M- } \\
\text { convex. }\end{array}$ & $\begin{array}{l}0.77 \text { (full sample); } 0.74 \text { (working } \\
\text { subsample). }\end{array}$ & $\begin{array}{l}-0.061 \text { (full sample); - } 0.064 \text { (working } \\
\text { subsample). }\end{array}$ \\
\hline Bourguignon and Nagnac (1990) & France & $\begin{array}{l}\text { French Labour Force Survey } 1985 \text {; sample } \\
\text { size } 1985 \text {, employed } 1175, \text { non employed } \\
817 \text {, all married, aged 18-60. }\end{array}$ & $\begin{array}{l}\text { H: w eekly normal hours; W: hourly net } \\
\text { wage, SS; Y: spouse's net income + } \\
\text { family allow ances. }\end{array}$ & Linear labour supply, convex. & $\begin{array}{l}\text { ML, ML random preferences; ML with fixed } \\
\text { costs, random preferencese. }\end{array}$ & 1;0.05. & $-0.3 ;-0.2$ \\
\hline Blundell et al. (1998) & United Kingdom & $\begin{array}{l}\text { British Family expenditure survey 1978- } \\
\text { 1992; sample size } 16781 \text { employed, } 7845 \\
\text { non employed, all married or cohabiting, } \\
\text { aged 20-50. }\end{array}$ & $\begin{array}{l}\text { H: usual w weeky hours and overtime; W: } \\
\text { usual pay and overtime over } \mathrm{H} ; \mathrm{Y} \text { : non } \\
\text { durable w eekly consumption minus } \\
\text { earnings. }\end{array}$ & $\begin{array}{l}\text { Semilog linear supply with controls for } \\
\text { children, education and cohort. }\end{array}$ & $\begin{array}{l}\text { Grouping instrumental variable (cohort and } \\
\text { educ time interacts), Difference in } \\
\text { difference estimator. }\end{array}$ & $\begin{array}{l}\text { No children: } 0.14 \text {; children 0-2: } 0.21 \text {; } \\
\text { children 3-4: } 0.37 \text {; chidren 5-10: } 0.13 \text {; } \\
\text { children } 11 \text { - : } 0.13 \text {. }\end{array}$ & $\begin{array}{l}\text { No children: } 0 \text {; children } 0-2:-0.19 \text {; children } \\
3-4:-0.17 \text {; children } 5-10:-0.10 \text {; children } 11 \\
\quad+:-0.06 \text {. }\end{array}$ \\
\hline Blundell et al. (1992) & United Kingdom & $\begin{array}{l}\text { British Family Expenditure Survey } 1981 \text { - } \\
1986 \text {; sample size } 1654 \text { total, } 67 \text { meet tax } \\
\text { selection criterai; Single with children; } \\
\text { excluding self employed. }\end{array}$ & $\begin{array}{l}\text { H: usual w weekly hours and overtime; W: } \\
\text { hourly wage; } Y \text { : consumption based. }\end{array}$ & $\begin{array}{l}\text { Heckman implicit labour supply function. } \\
\text { Linear budget constraint. }\end{array}$ & $\begin{array}{l}\text { Linear labour supply, budget constraint } \\
\text { only. }\end{array}$ & $\begin{array}{l}\text { Tax selected: } 0.16 \text {; complete: } 0.34 ; \text { tax } \\
\text { selected and grouping: } 0.14 \text {. }\end{array}$ & not available. \\
\hline Colombino and Del Boca (1990) & thaly & $\begin{array}{l}\text { Turin Survey of couples } 1979 \text {, sample size } \\
338 \text { employed, } 494 \text { non employed, married. }\end{array}$ & $\begin{array}{l}\text { H: yearly hours: } W \text { : hourly wage, SS; } Y \text { : } \\
\text { total net non labour rincome. }\end{array}$ & Linear labour supply, convex. & ML-convex ; Linear labour supply convex. & $\begin{array}{l}1.18 \text { (total): } 0.64 \text { (participants), } 0.54 \\
\text { (hours). }\end{array}$ & $\begin{array}{c}-0.66 \text { (total): }:-0.44 \text { (participantis), }-0.22 \\
\text { (hours). }\end{array}$ \\
\hline Deevereux (2004) & United States & $\begin{array}{l}\text { US Census of Population. Married couples. } \\
\text { Age 21-60. sample size } 1266752 \text {, total, } 62 \\
\text { percent participation rate (1980); sample } \\
\text { size } 1271 \text { 2715, total, } 74 \text { percent } \\
\text { participation rate (1990). }\end{array}$ & $\begin{array}{l}\text { H: annual hours; W: hourly wage; Y: non } \\
2 \text { labour income. }\end{array}$ & $\begin{array}{l}\text { Log-linear labour supply model where } \\
\text { hours w orked depend on w age, spouse } \\
\text { wage, income and other characteristics. } \\
\text { First differenced. }\end{array}$ & $\begin{array}{l}\text { Grouped tw } 0 \text {-stage least squares } \\
\text { estimator. Group fixed effects and error } \\
\text { correlation with education and age of } \\
\text { individuals. }\end{array}$ & 0.17 (median). & not available. \\
\hline Hausman (1981) & United States & $\begin{array}{l}\text { US Panel Study of Income Dynamics 1975; } \\
\text { size } 575 \text { participants, } 510 \text { non participants, } \\
\text { all married. }\end{array}$ & $\begin{array}{l}\text { H: annual hours; W: directly reported } \\
\text { hourly wage rates; Y: other income } \\
\text { assuming 8\% return to financial assets. }\end{array}$ & $\begin{array}{l}\text { Linear labour supply, convex and non } \\
\text { convex (fixed costs). }\end{array}$ & $\begin{array}{l}\text { ML-convex, random preferences; M-fixed } \\
\text { costs, random preferences (on income } \\
\text { coefficient). }\end{array}$ & $0.995 ; 0.906$ & $-0.121 ;-0.132$. \\
\hline
\end{tabular}


Table 2. Microeconomic literature: Empirical labour supply models, women (continued)

\begin{tabular}{|c|c|c|c|c|c|c|c|}
\hline Study & Country & Sample and estimation period & Variables used & Labour supply model & Estimation method & Uncompensated wage elasticity $^{1}$ & Income elasticity \\
\hline Kaiser et al. (1992) & Germany & $\begin{array}{l}\text { German Socioeconomic Panel 1983; } \\
\text { sample size } 1076 \text { employed, 2284 non } \\
\text { employed, married, non retired. }\end{array}$ & $\begin{array}{l}\text { H: annual hours; W: hourly wage, SS; Y: } \\
\text { income from rents capital income and } \\
\text { transfer payments. }\end{array}$ & $\begin{array}{l}\text { Linear labour supply, convex and non } \\
\text { convex (fixed costs). }\end{array}$ & ML-convex. & 1.04 & -0.18 . \\
\hline Kuismanen (1997) & Finland & $\begin{array}{l}\text { Finnish Labour Force Survey, } 1986 \text {, sample } \\
\text { size } 1541 \text { employed, } 485 \text { non employed, all } \\
\text { married, aged 25-60, tax register data. }\end{array}$ & $\begin{array}{l}\text { H: yearly hours; W: hourly wage, SS; Y: } \\
\text { I income from rents, dividiens, capital } \\
\text { income, etc.. }\end{array}$ & Semil-log labour supply, convex. & $\begin{array}{l}\text { ML-convex fixed preferences; ML-convex, } \\
\text { randompreferenences. }\end{array}$ & $-0.01 ; 0.01$ & $0.27 ; 0.11$ \\
\hline Kuroda and Yamamoto (2007) ${ }^{3}$ & Japan & $\begin{array}{l}\text { Semi-aggregated dataset by prefecture, } \\
\text { age group, and sex from the Survey on } \\
\text { Time Use and Leisure Activities and the } \\
\text { basic Survey on Wage Structure. Sample } \\
\text { period is } 1990 \text { ( (pooled cross sections- } \\
\text { 1992 } 2002 \text {, "Sample B"). }\end{array}$ & $\begin{array}{l}\text { H: total man-hour labour supply; W: Hourly } \\
\text { w age rate based on annual earnings. }\end{array}$ & $\begin{array}{l}\text { Life-cycle model, estimation of a Frish } \\
\text { (intertemporal) elasticity. }\end{array}$ & $\begin{array}{l}\text { Cross section estimation of a dynamic } \\
\text { model on grouped data ; Instrumental } \\
\text { variables. }\end{array}$ & $\begin{array}{l}1.26 \text { (extensive and intensive margin); } 0.13 \\
\text { (intensive margin). }\end{array}$ & \\
\hline Rose-Birch (2005) & Australia & $\begin{array}{l}\text { Meta-analysis on a complete review of } \\
\text { empirical studies using Australian data to } \\
\text { estimate women labour supply functions. }\end{array}$ & - & . & - & $\begin{array}{l}\text { Mean: } 0.33 \text { (25 studies); Data from } 1991 \\
\text { onwwards ( } 4 \text { studies): } 0.67\end{array}$ & Mean: -0.83 (13 studies). \\
\hline Smith and Stelcner (1988) & Canada & $\begin{array}{l}1981 \text { Canada Census. } 2851 \text { married } \\
\text { couples with wives aged 20-54. }\end{array}$ & $\begin{array}{l}\text { H: annual hours; } W \text { : hourly wage rate; } Y \text { : } \\
\text { vitual income (computed using data on tax } \\
\text { returns). }\end{array}$ & $\begin{array}{l}\text { Linear labour supply based on constrained } \\
x \text { choices on leisure and consumption. }\end{array}$ & Instrumental variables and selection. & $\begin{array}{l}\text { Age group: 20-34: 0.149, 35-24: 0.028; 20- } \\
\text { 54: } 0.100 \text {. }\end{array}$ & $\begin{array}{l}\text { Age group: 20-34:-0.184, 35-24:-0.077; } \\
\text { 20-54:-0.116. }\end{array}$ \\
\hline Triest $(1990)^{2}$ & United States & $\begin{array}{l}\text { US Panel Study of Income Dynamics 1975; } \\
\text { size } 715 \text { employed, } 485 \text { non employed, all } \\
\text { married, aged 25-60. }\end{array}$ & $\begin{array}{l}\text { H: annual hours; W: average hourly wage, } \\
\text { SS; Y: rent, interest, dividends, etc... }\end{array}$ & $\begin{array}{l}\text { Linear labour supply, convex and non } \\
\text { convex. }\end{array}$ & $\begin{array}{l}\text { Full sample: ML convex, random } \\
\text { preferences (on income coefficient); } \\
\text { Workers only: M-convex, random } \\
\text { preferences (on income ceefficient) }\end{array}$ & 0.97 & -0.33 \\
\hline Van Soest te al. (1990) & Netherlands & $\begin{array}{l}\text { Dutch Strategic Labour Market Research } \\
\text { Survey } 1985 \text {, sample size } 331 \text { particicants, } \\
470 \text { non participants, employed, } 48 \text { non } \\
\text { emoloyed, all married. }\end{array}$ & $\begin{array}{l}\text { H: average weekly hours; W: net hourly } \\
\text { wage, SS; Y: other incomes. }\end{array}$ & Linear labour supply, convex. & ML-convex. & 0.79 & -0.23 \\
\hline
\end{tabular}

Variables: H: hours; W: wage; Y: :income; SS means that wages are predicted via linear selectivity adjusted regression. Estimation method: ML: maximum likelihood

1. Figures separated by semi-colon refer to estimates based on different methodologies.

2. Easticities are evaluated at the observed mean w age and virtual income of participants

. This article estimales a Firsch elasticity, based on a dynamic model, contrary to the other articles review ed here.

Note: $\mathrm{h}$ this context, comparison has to be undertaken with great caution. See the original articles for detalls. 


\begin{tabular}{|c|c|c|c|c|c|c|c|}
\hline Study & Country & Sample and estimation period & Variables used & Labour supply model & Estimation method & $\begin{array}{l}\text { Uncompensated wage } \\
\text { elasticity }^{1}\end{array}$ & Income elasticity \\
\hline Blau and Kahn (2005) & United States & $\begin{array}{l}\text { March Current Population Survey } \\
\text { data, period: } 1980-1990-2000 \text {. } \\
\text { Sample: married individuals aged 25- } \\
54 \text { with a } 25-54 \text { spouse present. } \\
\text { Employment rate in 1970: } 0.97 \text {; in } \\
\text { 1999: } 0.96 \text {. }\end{array}$ & $\begin{array}{l}\text { H: annual hours; } \mathrm{W} \text { : annual earnings/ } \\
\text { annual work hours for wage and } \\
\text { salary workers; } Y \text { : income from } \\
\text { assets, including interest, dividend, } \\
\text { and rental income. Spouse's wage is } \\
\text { included. }\end{array}$ & $\begin{array}{l}\text { Linear supply model extended to } \\
\text { include spouse's wage. }\end{array}$ & Instrumental variables and selection. & 0.07 (median). & 0.00 (median). \\
\hline Blomquist (1983) & Sweden & $\begin{array}{l}\text { Swedish Level of Living Survey 1974; } \\
\text { sample size 688; employed; married; } \\
\text { age 25-55. }\end{array}$ & $\begin{array}{l}\text { H: annual hours; } W \text { : directly } \\
\text { observed; } Y \text { : spouse's net income + } \\
\text { family allowances + net capital } \\
\text { income. }\end{array}$ & Linear labour supply, convex. & $\begin{array}{l}\text { ML, ML random preferences (on } \\
\text { income coefficient). }\end{array}$ & 0.08 & $-0.03 ;-0.04$ \\
\hline Blundell and Walker (1986) & United Kingdom & $\begin{array}{l}\text { British Family Expenditure Survey } \\
\text { 1980; sample size 1378; all employed } \\
\text { married, aged 18-59. }\end{array}$ & $\begin{array}{l}\text { H: usual weekly hours; } W \text { : weekly } \\
\text { earnings; } Y \text { :consumption based two- } \\
\text { stage budgeting. }\end{array}$ & Gorman polar form/translog, convex. & ML convex, random preferences. & 0.024 & -0.287 \\
\hline Devereux (2004) & United States & $\begin{array}{l}\text { US Census of Population. Married } \\
\text { couples. Age 21-60, all employed. }\end{array}$ & $\begin{array}{l}\text { H: annual hours; } W \text { : hourly wage; } Y \text { : } \\
\text { non labour income. }\end{array}$ & $\begin{array}{l}\text { Log-linear labour supply model where } \\
\text { hours worked depend on wage, } \\
\text { spouse wage, income and other } \\
\text { characteristics. First differenced. }\end{array}$ & $\begin{array}{l}\text { Grouped two-stage least squares } \\
\text { estimator. Group fixed effects and } \\
\text { error correlation with education and } \\
\text { age of individuals. }\end{array}$ & 0.00 (median). & not available. \\
\hline Hausman (1981) & United States & $\begin{array}{l}\text { US Panel Study of Income Dynamics } \\
\text { 1975; size 1085; all employed, } \\
\text { married, aged 25-55. }\end{array}$ & $\begin{array}{l}\text { H: annual hours; } \mathrm{W} \text { : directly reported } \\
\text { hourly wage rates; } \mathrm{Y} \text { : other income } \\
\text { assuming } 8 \% \text { return to financial } \\
\text { assets. }\end{array}$ & Linear labour supply. & $\begin{array}{l}\text { ML, ML random preferences (on } \\
\text { income coefficient). }\end{array}$ & $0.00 ; 0.03$ & $-0.95 ;-1.03$ \\
\hline Kuroda and Yamamoto (2007) & Japan & $\begin{array}{l}\text { Semi-aggregated dataset by } \\
\text { prefecture, age group, and sex from } \\
\text { the Survey on Time Use and Leisure } \\
\text { Activities and the Basic Survey on } \\
\text { Wage structure. Sample period is } \\
\text { 1990s (pooled cross sections - } \\
\text { 1992/2002). }\end{array}$ & $\begin{array}{l}\text { H: total man-hour labour supply; W: } \\
\text { Hourly wage rate based on annual } \\
\text { earnings. }\end{array}$ & $\begin{array}{l}\text { Life-cycle model, estimation of a Frish } \\
\text { (intertemporal) elasticity. }\end{array}$ & $\begin{array}{l}\text { Cross section estimation of a } \\
\text { dynamic model on grouped data; } \\
\text { Instrumental variables. }\end{array}$ & $\begin{array}{l}0.69 \text { (extensive and } \\
\text { intensive margin); } 0.14 \\
\text { (intensive margin). }\end{array}$ & - \\
\hline Ma Curdy et al. (1990) & United States & $\begin{array}{l}\text { US Panel Study of Income Dynamics } \\
\text { 1975; size 1017; all employed, } \\
\text { married, aged 25-55. }\end{array}$ & $\begin{array}{l}\text { H: annual hours; } W \text { : average hourly } \\
\text { wage; } Y \text { : rent, interest, dividends, } \\
\text { etc... }\end{array}$ & $\begin{array}{l}\text { Linear labour supply, convex and non } \\
\text { convex. }\end{array}$ & $\begin{array}{l}\text { ML convex, random preferences (on } \\
\text { income coefficient). }\end{array}$ & 0.00 & -0.01 \\
\hline Van Soest et al. (1990) & Netherlands & $\begin{array}{l}\text { Dutch Strategic Labour Market } \\
\text { Research Survey } 1985, \text { sample size } \\
801, \text { employed, } 48 \text { non employed. }\end{array}$ & $\begin{array}{l}\mathrm{H} \text { : average weekly hours; } \mathrm{W} \text { : net } \\
\text { hourly wage; } \mathrm{Y} \text { : other incomes. }\end{array}$ & Linear labour supply, convex. & ML convex. & 0.12 & -0.01 \\
\hline
\end{tabular}

Variables: H: hours; W: wage; Y: income. Estimation method: ML: maximum likelihood.

1. Figures separated by semi-colon refer to estimates based on different methodologies.

2. This article estimates a Frisch elasticity, based on a dynamic model, contrary to the other articles reviewed here.

Note: in this context, comparison has to be undertaken with great caution. See the original articles for details. 


\subsection{The issue: analyse the determinants of the intensive margin of labour supply}

28. The objective of the present empirical analysis is to estimate the impact of taxes and other policies on the labour supply of working individuals, controlling for the participation decision. In this context, the central question raised by this work can be summarised as follows: is taxation important for explaining cross-country differences in hours worked, over and beyond its importance for explaining crosscountry differences in employment rates?

29. This choice is motivated by the fact that the impact of the policies on the extensive margin of labour supply has been the object of a voluminous empirical literature, which has delivered a number of strong policy implications. This is true both at the cross-country level (see e.g. Bassanini and Duval, 2006, Jaumotte, 2003), but also, as mentioned in the previous section, in the microeconomic literature (Aaberge et al. 1999, Arrufat and Zabalza, 1986, Pencavel, 1998, Brewer and Browne, 2006, for women; Aaberge et al. 1999, Ransom, 1987, for men).

30. The present analysis is close to cross-country analysis in spirit to the extent that it investigates the impact of differences in policies on differences in hours worked among OECD countries. It, however, departs from this literature in several respects, and can be characterised as a "quasi"-microeconomic approach to labour supply:

- The present work explicitly disentangles the impact of policies on the intensive versus extensive margin of labour supply, contrary to the bulk of the cross-country literature.

- The empirical analysis tackles the endogeneity of the employment rate with respect to hours worked. In terms of labour supply, the participation decision cannot be considered independently from the decision on the number of hours supplied in the labour market (or, most probably, from the decision to work part-time or full-time). In terms of labour demand, the analysis considers the possibility that employers allocate total labour utilisation across extensive (hiring and firing) and intensive (hours worked of employed individuals) margins.

- By adopting a semi-aggregated approach, the empirical work allows to look more closely at behaviour of different groups of the labour force among OECD countries.

31. Despite a number of limitations (see below), this work intends to reconcile the somewhat contradictory results between microeconomic models of individual labour supply — delivering quite modest responsiveness of hours of work to taxation - and macroeconomic estimates of "aggregate" labour supply elasticities - implying significant disincentives effect of taxation on labour supply. ${ }^{13}$ This is

13. Note that there is now an emerging consensus - although on a pure theoretical perspective - among economists on the rationale beyond the divergence between micro and macro estimates. Two explanations are given for understanding this divergence. First, as discussed above, the elasticity of hours worked with regard to tax rates is very much dependent on how tax revenues are spent, as discussed above, (see discussion in Alesina et al. 2005, and Rogerson, 2006). Prescott assumes that all revenues to household take the form of a lump-sum transfer. Rogerson (2006) shows that if higher taxes subsidise day care for individuals who work, then the effect on hours of work will be less than under a lump-sum transfer case in which the size of the transfer is unaffected by the amount of labour supplied. This pattern of government spending would explain why while tax rates are high in Scandinavia, hours worked are higher than in continental Europe. Note however that this only operates through the extensive margin of the labour supply. The elasticities referred to here do not disentangle the extensive and intensive margin. Second, recent general equilibrium models show that the slope of the aggregate labour supply curve can 
done by opting for an intermediate, or semi-aggregated framework, which allows overcoming some of the limitations attached to macroeconomic and microeconomic approaches:

- Macroeconomic estimates suffer from aggregation and measurement error bias, both for hours worked, and, importantly, for aggregate measures of taxation.

- Microeconomic estimates are based on behavioural models but rely on single countries' data. The few available cross-country analyses are either based on micro-simulation techniques (see Immervol et al. 2007) or do not rely on cross-country empirical identification strategies (Geiner and Steiner, 2007). ${ }^{14}$

- The impact of working-time regulations and labour and product market institutions is not investigated in microeconomic studies (partly due to their country-specific nature). It is rarely analysed in cross-country empirical work on average hours worked; even when it is done, however, the impact is not disentangled between intensive and extensive margins. ${ }^{15}$

\subsection{The approach: from aggregate to semi-aggregate data}

32. This study attempts to reconcile micro and macro approaches by using cross-country analysis on semi-aggregated data. On the one hand, labour force surveys allow analysing the behaviour of different segments of the labour force, characterised by specific socio-economic characteristics and heterogeneous behaviour. The delimitation of these specific sub-groups is dictated both by data constraints and by results of the microeconomic literature, as summarised above. Gender, marital status and education are considered as basic building blocks of the disaggregate analysis. In turn, these groups have a different weight in the aggregate labour force, allowing to control for the compositional effects reflected in cross-country aggregate analyses.

33. On the other hand, disaggregated taxation models allow to build group-specific taxation indicators. This methodology is far from the microeconomics tradition, as it is not able to draw individual budget constraints. However, it departs from the previous cross-country work insofar as it allows inference to be based not only on cross-country variation in taxes and hours worked, but also on cross-country and cross-group variation in hours worked. Importantly, and contrary to most of the previous literature, this work relies on marginal, as opposed to average, tax rates, thus reflecting the relevant theoretical underpinnings of labour supply decisions.

significantly depart from the micro elasticity. This is the case in Chang and Kim (2005), in an economy where individuals are subject to idiosyncratic shocks to wages and labour is indivisible. This finding is most relevant in Rogerson and Wallenius (2007), who develop a general equilibrium life-cycle model of labour supply that incorporates both the intensive and extensive margin of labour supply. The authors find that micro and macro elasticities are virtually unrelated and that macro elasticities are large.

14. Geiner and Steiner (2007) use cross-country microeconomic data on female employment and working hours from the ECHP for four European countries which they identify as featuring distinct "institutional regimes". They do not, however, directly test the impact of differences in "institutional regimes" on differences in hours worked across those countries but suggest it through comparative analysis.

15. Some exceptions are worth mentioning. Buddelmeyer et al. (2004) analyse the determinants of part-time work in EU countries, and in this context, investigate the impact of labour and product market regulations. Faggio and Nickell (2007) also report some panel regressions on the impact of labour market institutions on average hours worked by employed individuals. The authors, however, do not focus on these issues in their paper. Fang and Rogerson (2007) provide a different approach by deriving the expected impact of taxes and other policies on the extensive versus the intensive margin of labour supply. The approach is purely theoretical, however, and the model is not confronted with cross-country data. 
34. Finally, cross-country data allow analysing the impact of time-varying policy settings (taxes, working-time regulations, labour and product market policies) on patterns of working hours. While these policies do not vary across groups, their impact is likely to be heterogeneous, based on individual characteristics and behaviour on the labour market. This approach is rarely used in the cross-country literature, ${ }^{16}$ it is practically absent in the cross-country literature on hours worked.

\subsection{Frontiers and limitations of the analysis}

35. While this work aims at countering the bias raised by cross-country estimates of average hours worked by adopting a "quasi"-microeconomic approach, it suffers from a number of limitations. The first and most important is that this analysis is not intended to estimate a structural labour supply model. This comes from the adoption of a cross-country approach, but it is also a consequence of the nature of the data:

- This analysis relies on semi-aggregated data computed from labour force surveys, as opposed to household or individual data, on which empirical labour supply models are based.

- There is no information on wages nor on unearned income in the labour force survey data used here, making it impossible to estimate income and substitution effects.

- This analysis estimates the impact of taxes on individuals who work, conditional on the impact of taxes on participation. While it addresses the simultaneity bias between employment and hours worked across the different segments of the labour force, it does not address the potential selection bias arising within each segment of the labour force. The use of instrumental variable techniques to tackle the endogeneity of the employment rate with respect to hours worked allows reducing selection bias based on observable variables (education, socioeconomic, demographic variables). It cannot address the potential bias arising from unobservable variables driving both the intensive and the extensive margin, the size and direction of which would be difficult to predict. $^{17}$

36. Working hours are identified with market work in the present empirical work. It will thus not be possible to investigate the determinants of the allocation of work between market and home production. Recent literature (Burda et al. 2007, Burda et al. 2006, Schettkat, 2003) makes use of time-use surveys to document differences across countries in the distribution of market versus household work. One important finding revealed by time-use surveys is that countries in continental Europe have a higher share of home production in total work compared to the United States. Labour taxation can potentially play a role in explaining cross-country differences in this division, and some recent descriptive evidence goes in that direction (Burda et al. 2006). Time-use surveys are only available for a few countries and on a crosssection basis, precluding their use in the econometric approach. Nevertheless, this limitation of the following analysis has to be kept in mind when interpreting the results.

16. For an assessment of the differential impact of policies by gender and age, see Bertola et al. (2002), Bassanini and Duval (2006), and Jimeno et al. (2002); for an assessment of the differential impact of policies by migration status, see Causa and Jean (2007).

17. While the size of the potential selection bias is difficult to assess in this context, comprehensive tests undertaken by Mroz (1987) in the context of female labour supply suggest that the selection bias problem appears to manifest itself primarily though the work experience variable. This variable is not contained in the present work. 


\subsection{The potential role of labour demand-side effects}

37. Labour supply theory, as briefly outlined above, establishes the amount of working hours a person desires to offer to the market at different wage rates. The impact of policies is interpreted in terms of labour supply behaviour. For instance, it is implicitly supposed that variations in labour taxes paid by employees and by employers are shifted into lower net wages, thus causing a labour supply reaction. ${ }^{18}$ The impact of other institutions, such as unionisation, is also interpreted in the literature through the lenses of a labour supply model in which it is implicitly assumed that the firm does not face any decision at the intensive margin. This partial equilibrium framework is, however, insufficient for building the theoretical underpinnings of the present work. Labour demand considerations, though not modelled explicitly, cannot be ignored. This section briefly outlines some of the relevant mechanisms at stake.

38. There are many reasons for supposing that the composition of total hours worked between workers and the average hours per worker matters and that the nature of the work schedule is important for the firm:

- Specialisation and division of labour in the enterprise gives rise to time complementarities among workers.

- Workers' productivity is not independent of hours worked per period, due to setup time of work activity, as well as reasons of fatigue and boredom. ${ }^{19}$

39. In a competitive situation, the firm extends both employment and hours to the point where their marginal costs equal their marginal products. Total costs of labour services can be modelled to include two components: direct wage costs, which depend on total hours worked, and "quasi-fixed costs, related to employment alone. These include search, hiring, and firing costs. Assuming separability, so that employment and hours per worker combine to produce labour services, allows to examine the nature of the split between hours and employment as an optimisation problem (see Rosen, 1978, and Hamermesh, 1993, for an exhaustive analysis on the trade-off between the extensive and the intensive margins of labour demand). It is thus possible to predict the consequences of changes in parameters, notably the level of fixed versus variable labour costs, on firms' decision.

40. The major proposition that can be established in this context is that the ratio of employment to hours in equilibrium is negatively related to the ratio of employment costs associated with each employee (hence "quasi -fixed" costs) to direct wage costs. Hence, policy-driven variations in a fixed or variable costs change the relative cost of employment and hours per person, implying substitution between hours and worker, at fixed level of labour services. ${ }^{20}$ However, these changes also affect the entire structure of costs in the firm and industry and hence shift the supply curve of the final product. Indirect effects of this type are called "scale effects" and can counteract substitution effects. In turn, the magnitude of the scale

18. See, inter alia, Daveri and Tabellini (2000).

19. Rosen (1978) incorporates these effects in a simple analytical framework: $i$ ) for one worker, productivity is S-shaped, reflecting first setup costs, then fatigue effects; and ii) for several workers, the model incorporates a penalty for lack of coordination in time worked, in the sense that for a given total number of hours, complementarities would make output larger if all employees worked the same hours.

20. Fang and Rogerson (2007), in a very recent article, also model an environment in which firms take decisions on both the intensive and the extensive margins. They propose a more complex modeling framework based on embedding a canonical model of labour supply in a standard matching model à la Pissarides. In their framework, only the substitution effect between the extensive and intensive margin is operative. The authors examine the implications of policies and institutions along these two margins in order to qualitatively distinguish between various explanations in hours worked across countries. 
effects depend on industry and firm characteristics (labour intensity, demand elasticity and, conversely, market power).

41. In this setting, while lower taxes on wages are expected to unambiguously increase the demand for hours (substitution and scale effects go in the same direction), the impact on employment depends on the strength of substitution (negative) versus scale (positive) effects. Conversely, the impact of a decrease in firing costs would imply an unambiguous positive effect on employment (substitution and scale effects go in the same direction), but an indeterminate effect on the demand for hours (once again, due to opposing scale and substitution effect). Positive effects on both margins can be expected in case of labour-intensive and (price) demand-elastic industries. This dependence on market structure also suggests the potential role for product market regulation to affect firms' hours- employment decision.

42. The simultaneous modelling of supply of, and demand for, hours per person can be approached in several ways. For the purpose of the following empirical analysis, the intuition given in Rosen (1978) can be particularly relevant: ${ }^{21}$

- There is a spectrum of closely interconnected markets geared to different work schedules: one market for long hours, one for full-time jobs, one for short hours jobs, and so on. Hence, workers cannot arbitrarily vary working hours on a given job, but must seek out a different job in a different market. If a firm desires to vary its working hours schedule, it must trade in a different market and employ different types of workers.

- In this context, policies and institutions can alter the incentives to operate in different hours markets - both from the perspective of the firm and the worker - and this would be reflected in variations in the proportion of part-time workers, hence contributing to the explanation of differences in average hours worked per employed. ${ }^{22}$

43. The impact of working-time regulations on hours worked should also be assessed in light of a labour supply and labour demand framework. Cahuc et al. (2006) provide a comprehensive analysis of the theory and the empirics of working-time reductions. The authors show that the effects of working-time reductions are very different in cases when the hourly wage is constant as opposed to the case when there is a wage compensation. In this latter, more realistic occurrence (the authors cite the French and German examples in this respect), collective bargaining models help to assess the influence of the institutional context on the choice of working hours and the efficiency of reducing working time. The models deliver the following predictions:

- Increases in unions bargaining power lead to lower working hours.

- Increases in product market competition lead to higher working hours.

21. This discussion omits the issue of whether individuals or firms are in disequilibrium with respect to their choice of working hours. Considering this possibility is out of the scope of the present work, and would require detailed individual-level data. For a discussion on the hours constraints and an estimation based on United Kingdom data, see Bryan (2006).

22. Buddelmeyer et al. (2004) suggest the influence of policy factors on the proportion of part-time work from a labour demand perspective. For example, they argue that the stringency of job protection may have two conflicting effects: $i$ ) it can encourage the use of part-time work so as to circumvent highly rigid employment legislation affecting full-time jobs; ii) it can affect adversely and directly part-time employment by strictly limiting its use. While the first effect would tend to decrease average hours worked, the second would have the opposite effect. 


\section{Cross-country analysis: who drives the aggregate relationship? A reassessment}

44. The goal of this section is to reassess the impact of policies, notably taxation, in explaining aggregate differences in average hours worked among employed persons across OECD countries. As mentioned above, a number of studies have pointed to the role of taxes in explaining aggregate differences in labour utilisation. Most of these have relied on average measures of labour taxation. As noted before, most of them have also not distinguished between the intensive and extensive margins of labour supply. This section attempts to fill those two gaps, therefore reassessing the aggregate impact on taxes on hours worked.

45. The relationship between aggregate measures of taxation and aggregate measures of hours worked is illustrated Figure 1, where annual average hours worked per employed person are plotted against three aggregate indicators: $i$ ) the average direct labour tax wedge for a one-earner family earning the average production worker wage (APW); ii) the ratio of the sum of direct taxes, social contributions and indirect taxes to GDP; and iii) an average indicator of marginal taxation for the second earner. ${ }^{23}$

46. These scatterplots illustrate the ambiguity of the aggregate relationship between taxes and hours worked. Indeed, as discussed earlier, microeconomic theory predicts conflicting income and substitution effects of taxes on labour supply. Furthermore, aggregation of taxes and hours worked might create measurement error, whose direction is difficult to predict. These figures indicate two main stylised facts:

- There appears to be no association between the tax wedge at the levels of APW earnings of the average production worker and annual average hours worked among employed individuals.

- While the cross-country scatterplot suggests a negative relationship between ex-post measures of taxation (the sum of direct and indirect taxes over GDP) and the intensive margin of labour supply, the most significant negative relationship is found when: $i$ ) an indicator of marginal taxation is used, as opposed to average taxation; and ii) an indicator of taxation of a second earner is used, as opposed to a first earner.

These simple scatterplots are consistent with the findings of the microeconomic literature concerning the importance of substitution effects, measured through the lenses of a marginal wage variable - for second earners - mostly identified with married women.

23. See below for definitions and sources. 
Figure 1. Annual average hours worked per person on employment and aggregate measures of taxation

A. Average tax wedge

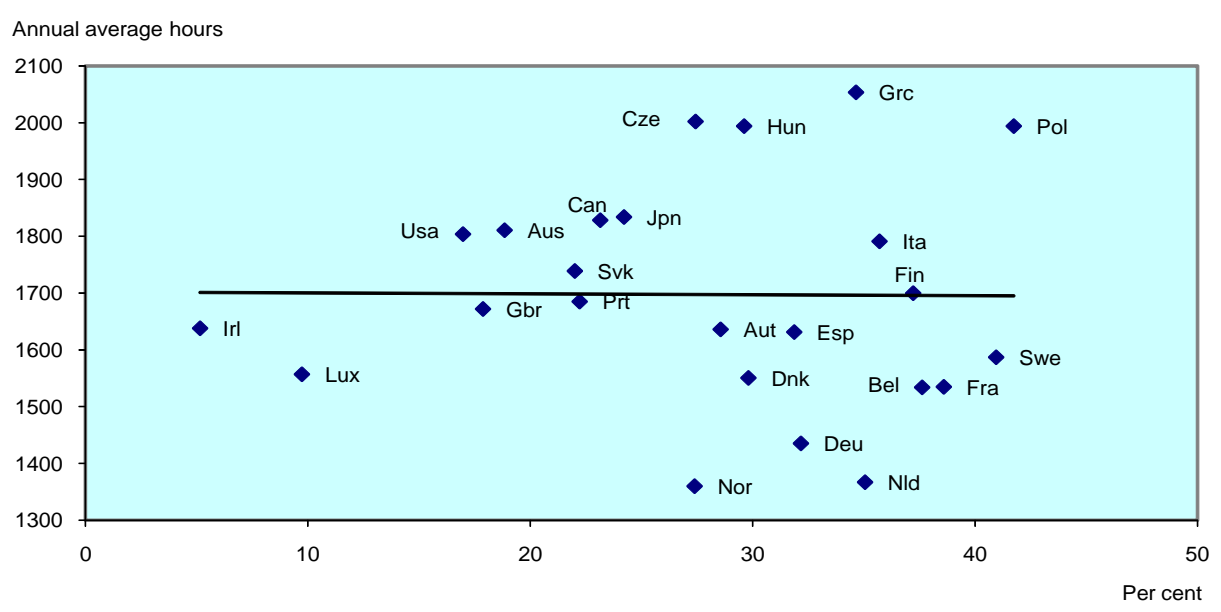

B. (Taxes and social contributions + indirect taxes) / GDP

Annual average hours

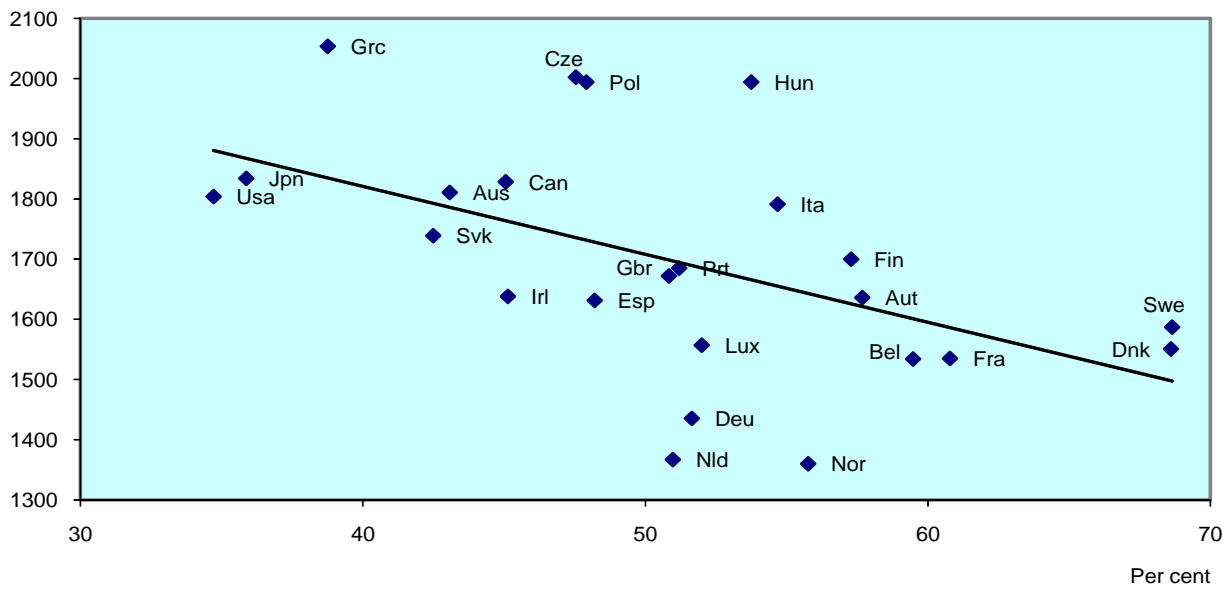

C. Average marginal tax wedge on second earner

Annual average hours

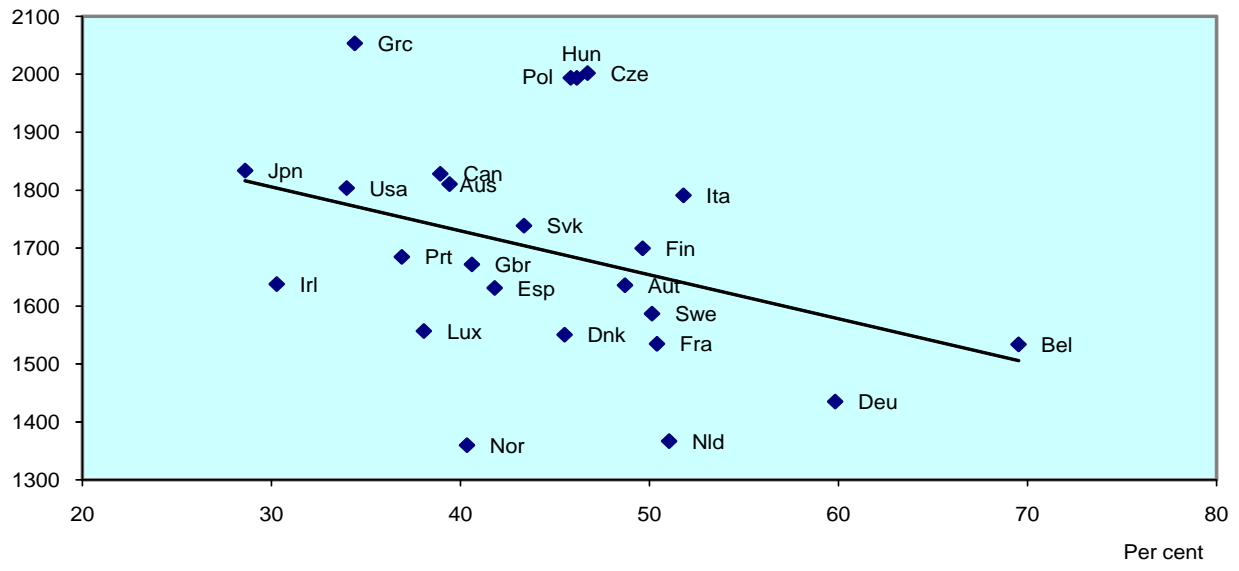

Note: Data refer to 2005

Source: Bassanini and Duval (2006); OECD Economic Outlook No 81; OECD Taxing Wages database. 


\section{ECO/WKP(2008)4}

\subsection{Empirical approach}

\subsubsection{Data sources and definitions}

47. The dependent variable in the econometric model is total annual hours worked divided by total persons in employment. The source for this variable is the OECD Employment Outlook (EmO) ${ }^{24}$

48. The explanatory variables can be divided into three categories:

1. Aggregate taxation indicators. This study makes use of the following variables:

- Average tax wedge for a one-earner family with APW earnings (from Bassanini and Duval, 2006).

- Ratio of the sum of taxes and social contributions to GDP, and ratio of the sum of direct taxes, social contributions and indirect taxes to GDP (from the Economic Outlook database).

- Average of marginal tax wedges on a second earner, based on OECD Taxing Wages models. ${ }^{25}$

2. Indicators of union power. This study uses the union density variable, defined as the share of workers affiliated to a trade union. The source is EmO updated by the 2006 Going for Growth publication.

3. The control variables included in the analysis are:

i) The employment ratio, defined as the number of employed persons as a share of the working-age population. The employment rate is included to control for the extensive margin of the labour supply decision, but also, as explained above, for labour demand effects. A higher employment rate generally implies a larger share of working women, young persons and older persons. As these segments of the population are more likely to work part-time than adult males, the coefficient of the employment rate is expected to be negative. The source is $E m O$.

ii) Income per capita defined as GDP in 2000 PPP USD divided by total population. If leisure is a normal good, a rise in income per capita is expected to reduce average hours worked. The source of this variable is the OECD Economic Outlook (EO).

24. A few changes were made to the original EmO series to improve cross-country comparability. The hours worked series for Canada and Japan reported in EmO are on a per job basis. An estimate of average hours per employed person was made by using the approximation: $\mathrm{AHW}=\mathrm{AHWJ} *(1+\mathrm{SMJ})$, where $\mathrm{AHW}=$ average hours worked per employed person; AHWJ = average hours worked per job; and SMJ = share of workers with multiple jobs. The source of SMJ data for Canada is Statistics Canada while for Japan it is the Japanese Ministry of Internal Affairs.

25. This variable is a simple average of marginal tax wedges on a second earner based on disaggregated tax indicators for several types of household and level of earnings. For further explanations, see next section and the appendix. 
iii) The output cycle indicator is the log of real GDP filtered through the Hodrick-Prescott filter. The smoothing parameter is set at 6.25 , as recommended by Raven and Uhlig (2002) for annual data. The output cycle is expected to influence average hours worked positively (labour hoarding, i.e. firms respond to cyclical fluctuations by adjusting the intensive margin of labour demand). The source for real GDP is $E O$.

49. The 21 countries included in the panel are: Australia, Austria, Belgium, Canada, Denmark, Finland, France, Germany, Greece, Ireland, Italy, Japan, the Netherlands, New Zealand, Norway, Portugal, Spain, Sweden, Switzerland, the United Kingdom and the United States. The estimation sample period is $1996-2003 .^{26}$

\subsubsection{Model specification}

50. The econometric model used in this section is:

$$
\ln \left(\boldsymbol{A H} \boldsymbol{W}_{c t}\right)=\alpha \boldsymbol{T A} \boldsymbol{X}_{1 c t}+\beta \boldsymbol{T A} \boldsymbol{X}_{2 c t}+\gamma \boldsymbol{E r}_{c t}+\lambda \boldsymbol{Z}_{c t}+\phi_{c}+\eta_{t}+\varepsilon_{c t}
$$

where $\mathrm{c}$ and $\mathrm{t}$ are country (when applicable) and year subscripts, respectively; AHW is average annual hours worked per person in employment; $\mathrm{TAX}_{1}$ is an indicator of average taxation, $\mathrm{TAX}_{2}$ is an indicator of marginal taxation, Er represents the employment rate, and $\mathrm{Z}$ represents a vector of other variables (union density; the other control variables listed above).

51. Country-fixed effects are modelled with country dummies and are expected to capture the average influence of country-specific omitted variables on average hours worked. Time effects are modelled through time dummies and should capture year-specific factors influencing hours worked that are common to all countries included in the analysis.

52. The employment rate is likely to be endogenous in this setting. Hence, the equation is also estimated by instrumental variable/two-stage least squares (IV/2SLS) methods. In all specifications, instruments are lagged average replacement rate of unemployment benefits, lagged employment protection legislation (EPL), lagged product market regulation (PMR) indicators, and the lagged cyclical position. Both OLS and IV estimations make use of heteroskedasticity and autocorrelation-consistent standard errors, taking account of the panel structure of the data. The results are presented in Table 4.

26. The sample is restricted to this short period in order to consistently interpret the aggregate and then the disaggregate results (next section) because, contrary to measures of average taxation, disaggregated measures of (second earners') marginal taxes are only available for this last decade from the Taxing Wages Database. 
Table 4. Econometric estimates - Aggregate results

\begin{tabular}{|c|c|c|c|c|c|c|c|c|}
\hline \multicolumn{9}{|c|}{ A. Aggregate results without country fixed effects } \\
\hline & OLS & OLS & OLS & IV & IV & OLS & OLS & IV \\
\hline & 1 & 2 & 3 & 4 & 5 & 6 & 7 & 8 \\
\hline Employment rate & {$[0.133]$} & {$[0.114]$} & [0.112] & {$[0.540]$} & {$[0.581]$} & [0.113] & {$[0.117]$} & {$[0.284]$} \\
\hline \multirow[t]{2}{*}{ Output gap } & -0.219 & 0.213 & 0.079 & 0.26 & 0.394 & -0.09 & & \\
\hline & [1.291] & {$[1.118]$} & {$[1.130]$} & {$[1.717]$} & [1.249] & [1.159] & & \\
\hline Average tax wedge & {$[0.110]$} & & & [0.223] & & & & \\
\hline \multirow[t]{2}{*}{ (Taxes and social contributions + Indirect taxes) / GDP } & & $-0.508^{\star \star \star}$ & & & & $-0.190^{*}$ & -0.175 & -0.173 \\
\hline & & {$[0.077]$} & & & & {$[0.108]$} & {$[0.106]$} & {$[0.117]$} \\
\hline \multirow[t]{2}{*}{ Taxes and social contributions / GDP } & & & $-0.715^{\star \star \star}$ & & $-0.730^{\star \star \star}$ & & & \\
\hline & & & {$[0.108]$} & & {$[0.148]$} & & & \\
\hline Average marginal tax wedge on second earner & & & & & & $-0.421^{\star \star \star}$ & $-0.467^{\star \star \star}$ & $-0.469^{\star \star \star}$ \\
\hline
\end{tabular}

B. Aggregate results with country fixed effects

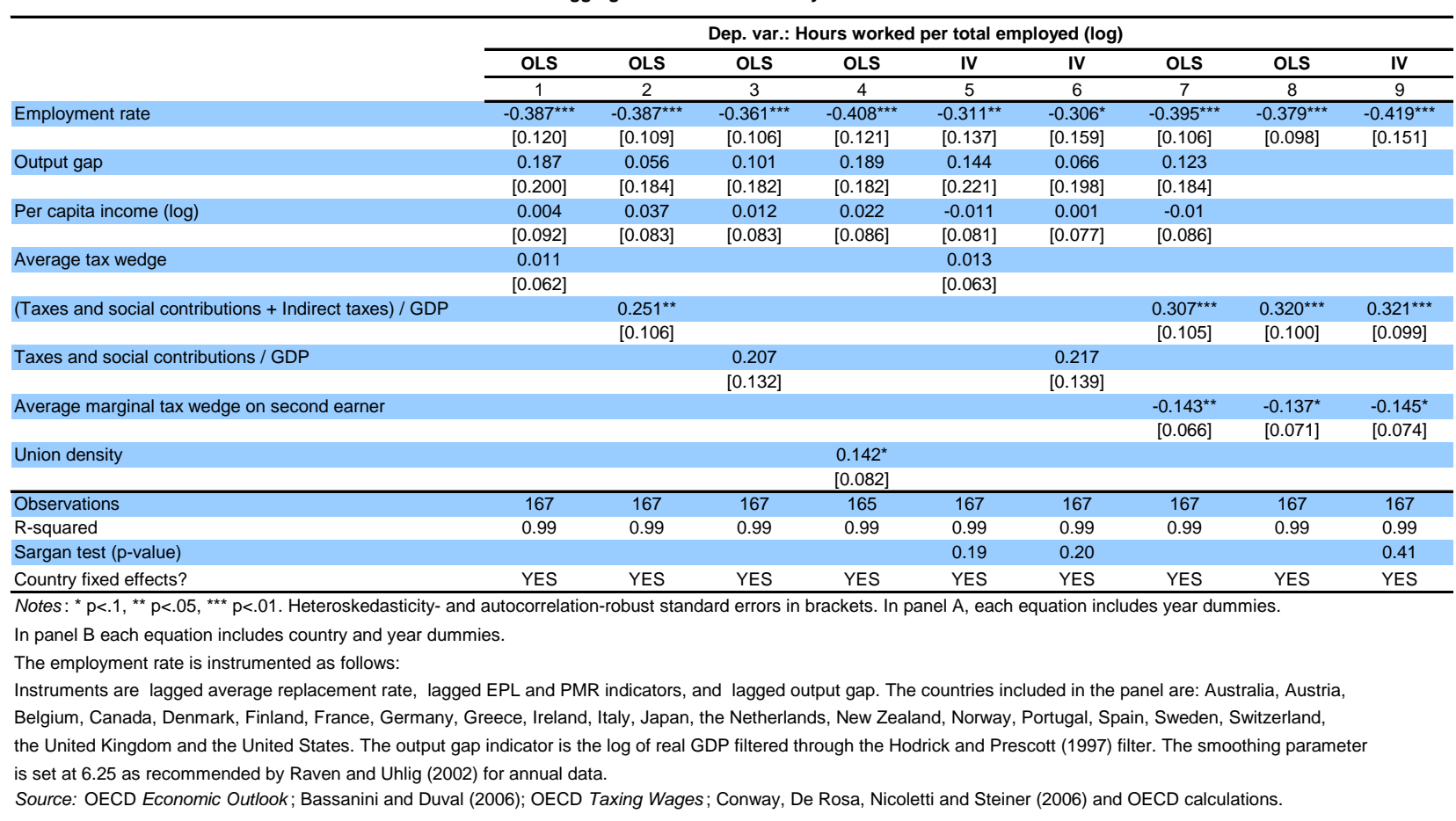

\subsection{The results: the second earner is key!}

53. Simple cross-country regressions of annual hours worked on average measures of taxation confirm findings in the literature of a negative relationship between the two variables. This result is robust to the use of either the average tax wedge on a one-earner family earning APW, or ex-post measure of taxation, such as the ratio of the sum of taxes, social contributions, and indirect taxes to GDP, or the ratio of the sum of taxes and social contributions to GDP (Table 4, Panel A, columns 1 to 3). Instrumentation of the employment rate (Panel A, columns 4 and 5) does not affect this finding. However, when adding to the basic specification an indicator of marginal taxation of the second earner, this negative relationship is weakened (column 6), and, in an instrumental variable specification, disappears (columns 7 and 8). In this 
latter case, indeed, while the impact of the marginal tax wedge on the second earner is negative and statistically significant, that of average taxation is positive and statistically insignificant. The omission of country-specific time-invariant features might, however, seriously bias the estimates, as is generally acknowledged in the cross-country literature. Therefore, it is crucial, in order to avoid having unobserved country-specific features to be driving the estimated relationships, to control for country-specific effects, hence relying on within-country variation in tax policy. This is done in panel B.

54. When country-specific effects are introduced in the specification, estimates of the impact of taxation on the intensive margin of labour supply confirm that average measures of taxation poorly reflect the potential disincentives to work longer hours. Indeed, average measures of taxation, in a cross-country panel context, are weakly related to average measures of hours of work: this is true both for the average tax wedge on a one-earner family with APW earning (Panel B, column 1), and for the ex-post measure of taxes and social contributions over GDP (Panel B, column 3), for which the estimated parameters are insignificant. This result is robust to instrumentation (Panel B, columns 5 and 6). The use of an alternative ex-post indicator, defined as the sum of taxes, social contributions and indirect taxes over GDP, gives a significant positive association with average hours worked, suggesting the fundamental ambiguities attached to the use of aggregate measures, as mentioned before (Panel B, column 2).

55. The fundamental result emerging from the estimates is that marginal taxation of the second earner is the channel through which the disincentives effect of high taxes are channelled into lower working hours. Indeed, introducing simultaneously a measure of average taxation and a measure of marginal taxation of the second earner delivers an intuitive result, suggesting "quasi"-income (average taxation, exhibiting a positive sign) and substitution (marginal taxation, exhibiting a negative sign) effects (column 7). This result is robust to instrumentation (column 8), and suppression of insignificant variables (column 9). ${ }^{27}$

56. The impact of union density is positive in this setting, contrary to some of the cross-country results presented above (column 4). Apart from the differences in methodology and in sample, which could go a long way towards explaining this discrepancy, one interpretation of this result is that aggregate measures of working hours mask important differences among various groups of the labour force, exhibiting varying behaviour and relationship with labour market institutions. In particular, it is not clear how hours of work by women are influenced by unions. ${ }^{28}$

57. The other explanatory variables have the expected signs. The negative coefficient of the employment rate would suggest that a trade-off could exist between the extensive and intensive margins of labour supply. Indeed, the share of part-time employment could rise as more marginal groups of workers participate to the labour force. Higher income per capita implies lower average hours worked, thus suggesting that leisure is a normal good as expected. However, the parameter estimated is not significant when country fixed effects are included. ${ }^{29}$ Similarly, an insignificant estimated parameter is found on the output gap indicator.

58. Aggregate estimates of the cross-country determinants of working hours confirm the need to go beyond average aggregates and to look at the heterogeneous behaviour of specific groups composing the labour force:

27. The variables in question are the output cycle and the income per capita.

28. The impact of this variable when country-specific effects are not controlled for is insignificant (not presented here). On this controversial topic, see Burgoon and Baxandall (2004).

29. This result might come from the fact that the identification relies on the within country variation across years, over a relatively short period of time. 
- The results suggest that the negative cross-country relationship between taxes and hours worked is not the reflection of some disincentives attached to average levels of taxation, but rather reflects the behavioural responsiveness of the second earner to marginal taxation. This argument calls for a careful analysis of working hours by the groups that the micro literature has identified as having the more elastic labour supply, notably women. The following empirical analysis will thus concentrate on the impact of marginal taxation, contrary to what the macroeconomic literature has done so far and more closely to microeconomic labour supply models (that focus on the marginal wage rate).

- This heterogeneity calls for a disaggregate assessment of the impact of labour and product market institutions and policies on hours worked by different segments of the labour force. In this context, the impact of working-time regulations and of other labour and product market policiesabsent from the aggregate analysis- will be explored in the disaggregate analysis. ${ }^{30}$

\section{Semi-aggregate analysis: an assessment of the role of taxes and other policies}

\subsection{Empirical approach}

59. The goal of this section is the identification of the impact of taxes, working-time regulations, and other labour and product market institutions on working hours, conditional on employment, for several groups of workers with different weights in the aggregate labour force. The approach is based on disaggregated information on hours and employment provided in labour force surveys for OECD countries, and disaggregated information on taxation computed from tax models in Taxing Wages.

\subsubsection{Data sources and definitions}

60. The dependent variable in the econometric model is usual weekly hours worked by employed individuals, for several groups of the labour force. ${ }^{31}$ The analysis is performed using European Labour Force Survey (ELFS) data for twenty European countries (Austria, Belgium, Czech Republic, Denmark, Finland, France, Germany, Greece, Hungary, Ireland, Italy, Luxembourg, ${ }^{32}$ the Netherlands, Norway, Poland, Portugal, Slovak Republic, Spain, Sweden, Switzerland, the United Kingdom), and Current Population Survey (CPS) data for the United States, over the period 1995-2005. ${ }^{33}$

61. Group-specific taxation variables - average and marginal tax wedges - are mapped into the socio-economic groups defined by the LFS data. The source of these variables is Taxing Wages models. The procedure can be summarised as follows: ${ }^{34}$

30. The aggregate analysis only explores the impact of union density because of the pervasive presence of this variable in the empirical cross-country literature. The role of working-time regulations and of other labour and product market policies is explored in the disaggregate analysis.

31. Due to data limitations, the disaggregated analysis uses usual weekly hours worked from labour force surveys as the dependent variable instead of annual hours worked as in the above aggregate analysis. Therefore, its scope is restricted to about half of the annual hours variation between Europe and the United States. This may imply that the impact of taxation (and regulatory policies) could be underestimated compared with corresponding estimates using annual hours worked as the dependent variable, if these regulations have an impact on the length of leave taken during the year beyond their impact on the work week.

32. Luxembourg is absent from estimates for women due to data limitations.

33. Other countries are absent from the disaggregate regressions due to data limitations.

34. The details of the procedure are described in the appendix. 
- Marginal (defined at the individual level) and average (defined at the household level) tax wedges are calculated for each per cent of gross earnings from $1 \%$ to $200 \%$ of the APW wage for first and second earners in the different household categories. ${ }^{35}$

- This information needs to be "semi-aggregated" in order to be used in statistical analysis. This is done by calculating simple averages of marginal and average tax wedges for first and second earners across the different household categories. ${ }^{36}$

- The data are mapped in the socio-economic groups identified in the LFS. At this stage, a number of assumptions are required, and they are discussed in detail in the appendix.

62. Due to data limitations, the taxation indicators used here present two important caveats, that have to be acknowledged upfront: ${ }^{37}$

1. The analysis does not incorporate marginal effective tax rates (METR). Social benefits, in particular housing benefits and social assistance, are not embedded in the tax models used here. However, the impact of in work benefits conditional on working a minimum of hours is imbedded in the empirical work done here to the extent that those benefits take the form of tax credits; this is the case of the most relevant "hours dependent" benefits programmes (such as the Working Families Tax Credit in the United Kingdom).

2. The data do not include the cost of childcare. Childcare-related tax reductions and cash benefits have been shown to be important determinants of women labour supply (Immervol and Barber, 2005). However, the indicators considered here take into account tax relieves and family cash transfers universally paid in respect of dependent children between 5 and 12 years of age who are attending school (see Taxing Wages, OECD, 2005a). ${ }^{38}$

The last section of the paper attempts to assess the potential for these factors to explain working hours differences between countries.

35. The different situations considered, as well as the selection of a minimum level of earnings across the different household categories, are discussed in the appendix.

36. An important issue is whether simple or weighted averages should be used. According to the latter approach, the weights should be assigned according to the underlying distribution of income (for a discussion on the possible methodologies, see Barro and Sahasakul (1983), and Stephenson (1998)) Applying this method to the current context is not easy as one would need information on the distribution of income by gender and family situation. This information is not available on a cross-country consistent basis. Besides, even if this was feasible, it remains to be seen whether it is desirable for the econometric analysis. In a model trying to find a link between average hours worked and tax rates, the distribution of income is likely to be endogenous (especially in a semi-disaggregated setting as used in this part). Thus, using the distribution of income to calculate an indicator of average marginal tax wedges could make the latter endogenous, thereby giving rise to econometric problems.

37. A third limitation can be mentioned, pertaining to the exclusion of indirect taxes from the tax wedges used in the disaggregate analysis. Though indirect taxes are also likely to influence the supply of hours worked - despite the fact that they are largely absent from most microeconometric analysis of labour supply their impact is excluded due to lack of data on indirect tax rates applicable to different population groups.

38. Not only the cost of childcare, but also a number of policies pertaining to the structure of the childcare system, such as school hours, are likely to play a role (see OECD, 2004b). Unfortunately, no cross-country, time-series dataset quantifying those structural features is available. 
63. The empirical work makes use of indicators measuring regulations governing weekly normal hours and overtime. In most countries, normal working hours are mandated by law. In countries where this is not the case, the analysis relies on normal hours as established by collective agreements.

64. The other policy variables used in this analysis include those that the labour market literature has identified as having a major impact on employment and participation patterns, but rarely analysed in the context of the intensive margin of labour supply: employment protection legislation (EPL) on regular contracts and the stringency of product market regulation. ${ }^{39}$ The role of union density on the determination of working hours is also investigated, in line with previous literature, but with a disaggregated approach, which allows to examine the differential impact of unionisation on specific labour market groups.

\subsubsection{The approach}

65. The analysis focuses on the impact of taxes, working-time regulations, and other labour and product market institutions on hours worked, conditional on employment, for several groups of workers, each having a different weight in the aggregate labour force.

66. This analysis considers a limited number of policy reforms having the potential to modify actual hours worked for some - or all — of the considered subgroups.

- Changes in the level of marginal tax rate facing individuals, leaving the average level of household taxation unchanged.

- Changes in working-time regulations and other policies governing labour and product markets.

While the first reform is mostly grounded on the idea of labour supply responsiveness to tax (dis) incentives, the second reforms, as argued above, can result from both demand and supply considerations.

67. In a nutshell, the question asked by the empirical analysis can be summarised as follows: can policies increase or decrease hours worked by employed individuals? The answer to this question schematically draws on two analytical pillars of the present work:

- Group-specific responsiveness of working hours, conditional on employment, with respect to marginal taxation and other policies.

- The composition of labour force within countries: if high responsiveness is limited to groups that have a low weight in the labour force, the aggregate impact will be small.

\subsubsection{Sample selection and groups definition}

68. The point of departure of the analysis is the prime-age labour force (i.e. 25-54 year-olds). Concentrating on prime-aged individuals allows discarding atypical working schedules for young and old individuals. The focus is on employees. This selection can be justified along several lines, the main reason

39. It is important to stress that part-time contracts are mostly regulated by the legislation on permanent contracts, hence justifying the use of this indicator as opposed to the indicator of EPL on temporary contracts (see Buddelmeyer et al. 2004).

40. Once again, childcare and school related indicators would have been useful here. They could not be included for data unavailability in a panel context. 
being that the taxation variables used in this work, based on OECD Taxing Wages models, only refer to employees in the definition of the reference worker.

69. The analysis is undertaken separately for males and females, as the literature has extensively documented the important gender differences in labour market supply behaviour. For each gender, the national labour market is divided into six segments (or groups), corresponding to marital status crossed by educational attainment (distinguishing three levels of skills).

\subsubsection{The impact of children: data issues and sample definition}

70. A further sub-division is considered as an extension, defined by the presence of children in the household. Data limitations prevent using this sub-division along the entire set of estimations, as would seem more appropriate for the purpose of this work. Acknowledging the shortcomings associated with this approach, the baseline analysis attempts to include the effects associated with the presence of children through specific assumptions embedded in the computation of the taxation indicators (see appendix). The estimates, thus, will be presented according to the following order:

- Baseline estimates of the impact of taxation on working hours, without specifically controlling for the presence of children in the household. This ensures a wide cross-country coverage.

- Baseline estimates of the impact of taxation on working hours, specifically controlling for the presence of children in the household. This ensures to minimise measurement error of both the dependent variable and the explanatory taxation variables (as child-related taxation is included in the indicators). However, this refinement comes at the cost of a considerable reduction in the country sample, notably for the Northern European countries. In particular, the following countries are excluded from this analysis: Finland, Sweden, Netherlands, Denmark, Norway, Ireland and Switzerland.

- Estimates of the impact of working-time regulations and other labour and product market policies, based on the wider cross-country coverage, thus omitting to control for the presence of children in the household. This choice is motivated by the simple argument that those policy settings do not vary across groups, contrary to taxation indicators; it is therefore important, for identification purposes, to rely on a wide — and representative — cross-country sample.

\subsubsection{Model specification and identification strategy}

71. The econometric specification aims at identifying the impact of taxation, working-time regulations, and other labour and product market policies on hours worked by specific sub-groups of the labour market, conditional on employment. The following baseline specification is adopted per each gender: ${ }^{41}$

$$
\begin{aligned}
& \ln \boldsymbol{H}_{c e m}(t)=\alpha+\lambda E r_{c e m}(t)+\delta \boldsymbol{m} t w_{c m}(t)+\phi a t w_{c m}(t)+\left[\omega M \cdot a t w_{c m}(t)+\chi E \cdot a t w_{c m}(t)\right] \\
& +E+\boldsymbol{T}+\boldsymbol{C}+\boldsymbol{u}_{c e m}(t)
\end{aligned}
$$

41. This equation does not include a specific subscript allowing to identify the presence of children in the household, as most of the empirical analysis cannot rely on this information. When the information on the presence of children is available, this criterion is used to redefine the groups along a further dimension. In this case, the number of groups is multiplied by a factor of two (depending on the presence of children). 
Subscript $c$ refers to country, subscript $e$ to education level, and subscript $m$ to marital status, and jointly define a labour market segment, whereas t refers to time. $E r$ is the group-specific employment rate, computed as a proportion of employed persons in the group-specific active and inactive population, thus encompassing both participation (hence labour supply) and (un)employment (hence labour demand) effects. atw and $m t w$ stand respectively for household average tax wedge and individual marginal tax wedge. E, M, C and T represent fixed-effects for educational attainment, marital status, country and time, respectively. The two interaction variables, M.atw and E.atw, capture, respectively, marital status and education-level-specific effects of average taxation, the interpretation of which will be discussed below. This equation is then augmented by a vector of regulations/institutions ( $\mathrm{Pol}$ with corresponding parameters $\beta)$ and associated interaction terms with education levels (Pol.E). These elements are entered either one at a time or jointly. The specification follows:

$$
\begin{aligned}
& \ln H_{c e m}(t)=\alpha+\lambda E r_{c e m}(t)+\beta P o l_{c}(t)+\delta m t w_{c m}(t)+\phi a t w_{c m}(t)+ \\
& {\left[\omega M \cdot a t w_{c m}(t)+\chi E \cdot a t w_{c m}(t)+\rho E \cdot P o l_{c}(t)\right]+E+T+C+u_{c e m}(t)}
\end{aligned}
$$

72. The parameters of interest are $\beta$ and $\delta$. The first parameter is interpreted as a semi-elasticity of the intensive margin of labour supply with respect to the marginal tax rate, conditional on the extensive margin. The microeconomic literature suggests that the parameter to be significantly negative, in particular for women. The second parameter is interpreted as the impact of other regulations and policies on hours worked, conditional on employment.

73. The baseline equation includes the average tax wedge, defined at the household level, along its interactions with education level and marital status. This variable is associated with three effects.

- A "traditional" positive income effect; if leisure is a normal good, an increase in the average level of taxation holding the marginal level of taxation unchanged, is expected to increase individual hours worked.

- Proxy for household wealth. Microeconomic studies use different variables measuring unearned income (e.g. spouse's income for married women, income from rents, capital income). In this setting, a higher level of household income taxation is a proxy for higher unearned income, typically for second earners. As documented in the microeconomic literature, this effect is expected to be negative. To control for the income effects associated with higher wealth, the average tax wedge is interacted with marital status and education level.

- Because it is defined at the household level, this variable may capture joint labour supply behaviour between spouses. Consistent with this approach, when the analysis is extended to control for the presence of children in the household, the average tax wedge is interacted with the binary variable associated with an indicator of the presence of children.

74. Because of multicollinearity between tax indicators in levels, it is not possible to interact both variables with country and time-invariant controls such as education and marital status. In this context, whereas the interacted average tax wedges are used as control variables, the marginal tax wedge is not interacted with education and marital status variables in order to maximise group variability for identification purposes. To summarise, the average tax wedge is a control variable and the focus for policy analysis is on the impact of the marginal tax wedge, closer to the traditional labour supply literature which focuses on marginal wages. 
75. The heterogeneity across groups is used both as a source of identification for policies that vary across groups (taxes) and, for policy analysis, as an aggregation device allowing to control for compositional effects arising when policies have differential impact on specific segments of the labour market. The panel structure of the data (where the individual is defined by the intersection of the country $x$ group dimension, e.g. $\mathrm{C} \times \mathrm{E} \times \mathrm{M}$ ) is taken into account by a robust cluster estimate of the variancecovariance matrix, allowing for arbitrary intra-group correlation of the error structure. This procedure yields conservative estimates of the standard errors. Regressions are weighted by the labour force of each segment in order to avoid segments with few individuals having a disproportionate impact on the estimated effect for the average worker. The reference individual in the estimation is a single employee with uppersecondary level of education.

76. The endogeneity of the employment rate is tackled though the use of an instrumental variable estimator, where the instruments cover both group-specific cross fixed effects whose impact on working hours occurs through the channel of the employment/participation decision, as well as policy instruments that the literature has identified as having a major impact on labour market outcomes. ${ }^{42}$ The instruments used in the baseline estimates are listed below: ${ }^{43}$

- Regression for hours worked by men: $i$ ) interaction of education level with marital status; ii) interaction of education level with the lagged average replacement rate of unemployment benefits. When the presence of children is considered, the instruments also include iii) an interaction of marital status with the presence of children.

- $\quad$ Regression for hours worked by women: $i$ ) interaction of education level with marital status; ii) interaction of education level with lagged employment protection legislation concerning regular contracts. When the presence of children is considered, the instruments also include iii) an interaction of education level with the presence of children.

\subsection{The results: the differential impact of taxes and policies across genders}

77. This section presents estimates of the impact of taxation, working-time regulations, and other labour and product market policies on working hours of different groups of the labour force, conditional on employment.

\subsubsection{Taxes matter! (for women...)}

78. As discussed earlier, the literature has stressed the higher responsiveness of labour supply at low earnings levels. ${ }^{44}$ This is supported by the patterns displayed in Figure 2: the relationship between marginal taxation and hours worked is flatter for higher educated women than for medium and lower educated women. ${ }^{45}$

42. In this case, the argument would mostly be demand driven. See, inter alia, Bassanini and Duval (2006).

43. The source of the policy variables is Bassanini and Duval (2006).

44. A recent strand of the literature for the US has shown that very high income earners might be much more responsive to tax rates than middle or middle-high income earners (see Saez (2003)). However, this phenomenon is concentrated at the very top of the distribution and thus for the purposes of this work, this high income elasticity should not affect the overall intensive elasticity used for high-educated individuals.

45. Education is here considered as a proxy for earnings. 
Figure 2. Weekly hours worked among employees and average marginal tax wedges - Women

A. Low educated

Weekly hours worked

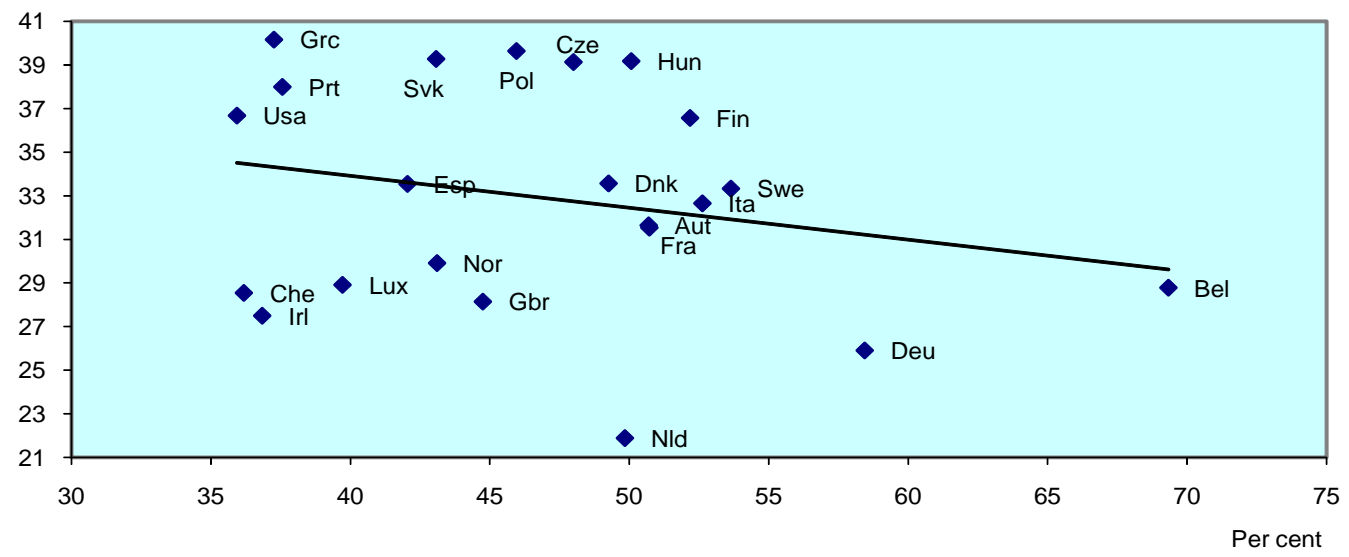

B. Medium educated

Weekly hours worked

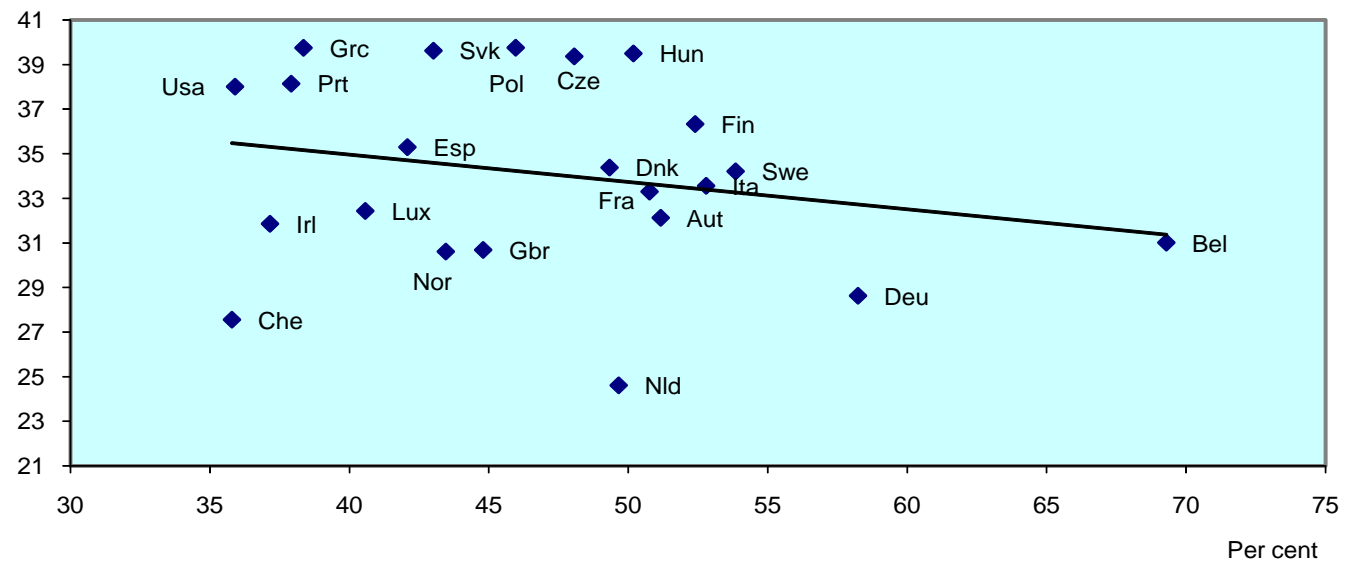

C. High educated

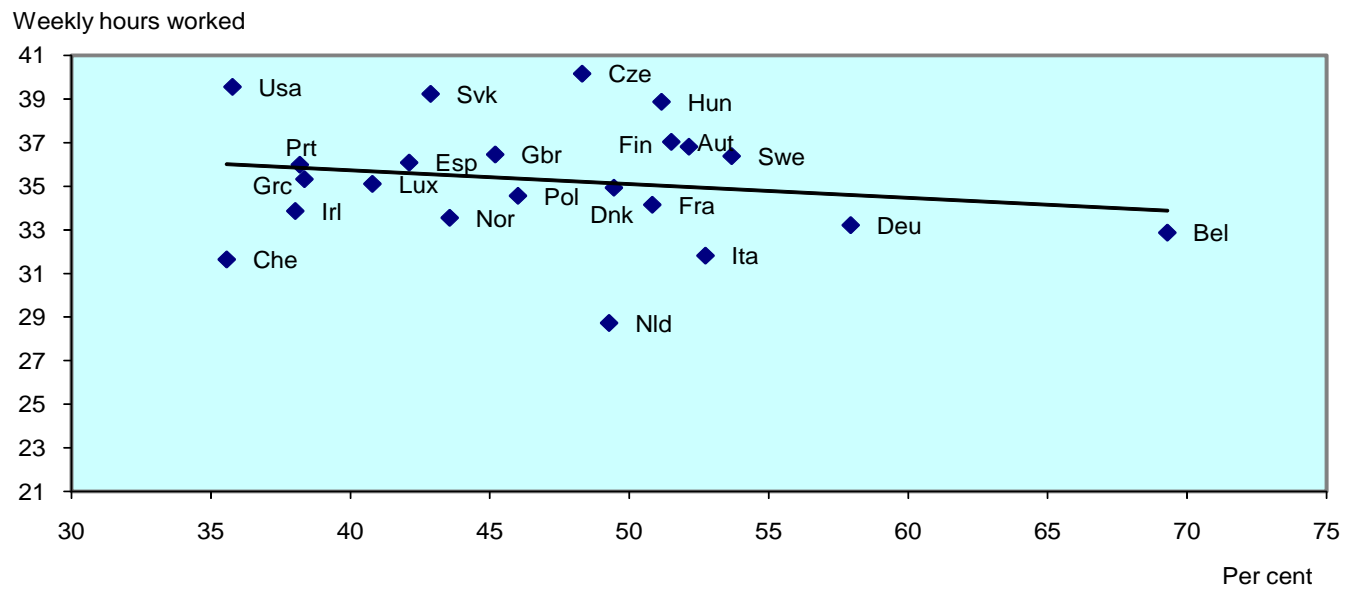

Notes: Prime-age labour force (25-54). See text and appendix for details on the computation of taxation indicators. Data refer to 2005.

Source: European Labour Force Survey; US Current Population Survey; OECD Taxing Wages database. 
79. The following table (Table 5) displays estimates of the baseline model obtained for the two samples: $i$ ) the sample in which the presence of children in the household is not taken into account, displaying complete cross-country coverage; ii) the sample in which it is possible to identify households with children, and conversely, include the corresponding tax indicators; in this case, however, the country coverage is narrow, and, notably, excludes Northern European countries.

Table 5. Econometric estimates - Taxes

\begin{tabular}{|c|c|c|c|c|}
\hline & \multicolumn{2}{|c|}{$\begin{array}{c}\text { Without taking account of the existence of } \\
\text { children }\end{array}$} & \multicolumn{2}{|c|}{ Taking account of the existence of children } \\
\hline & Men & Women & Men & Women \\
\hline & 1 & 2 & 3 & 4 \\
\hline \multirow[t]{2}{*}{ Employment rate } & 0.001 & -0.001 & 0.000 & 0.001 \\
\hline & {$[0.002]$} & [0.005] & {$[0.001]$} & [0.002] \\
\hline \multirow[t]{2}{*}{ Low education } & -0.043 & -0.103 & $-0.035^{*}$ & -0.087 \\
\hline & [0.027] & {$[0.108]$} & {$[0.019]$} & {$[0.079]$} \\
\hline \multirow[t]{2}{*}{ High education } & $0.054^{\star \star \star}$ & $0.095^{*}$ & $0.049^{\star \star *}$ & 0.065 \\
\hline & [0.015] & {$[0.057]$} & {$[0.011]$} & {$[0.044]$} \\
\hline \multirow[t]{2}{*}{ Married } & $0.072^{\star \star \star}$ & -0.138 & $0.057^{* \star *}$ & $-0.112^{* \star}$ \\
\hline & [0.015] & [0.084] & {$[0.010]$} & [0.053] \\
\hline \multirow[t]{2}{*}{ With children } & & & 0.007 & -0.061 \\
\hline & & & {$[0.014]$} & [0.052] \\
\hline \multirow[t]{2}{*}{ Average tax wedge } & 0.08 & $-0.703^{\star \star \star}$ & 0.004 & 0.088 \\
\hline & {$[0.083]$} & {$[0.268]$} & {$[0.068]$} & [0.201] \\
\hline \multirow[t]{2}{*}{ Marginal tax wedge } & $-0.136^{* \star}$ & $-0.730^{* \star *}$ & -0.084 & $-0.812^{\star \star \star}$ \\
\hline & {$[0.060]$} & {$[0.224]$} & {$[0.055]$} & {$[0.215]$} \\
\hline \multirow[t]{2}{*}{ Interaction married $\mathrm{x}$ average tax wedge } & $-0.151^{\star \star}$ & 0.089 & $-0.101^{\star *}$ & 0.127 \\
\hline & {$[0.068]$} & [0.149] & {$[0.046]$} & {$[0.126]$} \\
\hline \multirow[t]{2}{*}{ Interaction low education $\mathrm{x}$ average tax wedge } & $0.136^{*}$ & 0.119 & $0.108^{* *}$ & 0.181 \\
\hline & {$[0.070]$} & [0.194] & {$[0.051]$} & {$[0.187]$} \\
\hline \multirow[t]{2}{*}{ Interaction high education $\mathrm{x}$ average tax wedge } & $-0.111^{*}$ & -0.154 & $-0.087^{*}$ & -0.159 \\
\hline & [0.059] & [0.166] & [0.048] & [0.139] \\
\hline \multirow[t]{2}{*}{ Interaction children $x$ average tax wedge } & & & -0.028 & -0.084 \\
\hline & & & {$[0.032]$} & [0.151] \\
\hline Observations & 984 & 1164 & 1236 & 1512 \\
\hline Sargan test (p-value) & 0.47 & 0.52 & 0.59 & 0.30 \\
\hline N_clusters & 132 & 126 & 180 & 168 \\
\hline \multicolumn{5}{|l|}{ Notes: ${ }^{*} \mathrm{p}<.1,{ }^{* \star} \mathrm{p}<.05,{ }^{* * *} \mathrm{p}<.01$} \\
\hline \multicolumn{5}{|c|}{$\begin{array}{l}\text { Each equation includes country and year dummies. Weighted estimation. Standard errors in brackets are clustered by country } x \text { marital status } x \text { education } \\
\text { ( } x \text { children in columns } 3 \text { and } 4 \text { ). The employment rate is instrumented as follows: } \\
\text { Equations for men: Instruments are marital status interacted with education level and lagged average replacement rate interacted with education level. }\end{array}$} \\
\hline \multicolumn{5}{|c|}{ In the equation controlling for the presence of children, additional instruments are interactions of marital status and presence of children. Equations for } \\
\hline \multicolumn{5}{|c|}{ women: Instruments are marital status interacted with education level and lagged EPL on regular contracts interacted with education level. } \\
\hline \multicolumn{5}{|c|}{ In the equation controlling for the presence of children, additional instruments are interactions of education level with the presence of children. } \\
\hline \multicolumn{5}{|c|}{ The countries included in the panel are: Austria, Belgium, Czech Republic, Denmark, Finland, France, Germany, Greece, Hungary, Ireland, Italy, } \\
\hline \multicolumn{5}{|c|}{ Luxembourg, the Netherlands, Norway, Poland, Portugal, Slovakia, Spain, Sweden, Switzerland, the United Kingdom and the United States. } \\
\hline \multirow{2}{*}{\multicolumn{5}{|c|}{$\begin{array}{l}\text { Differences in the number of observations between men and women estimates are due to the absence of Luxembourg in equations for women. This absence } \\
\text { is caused by lacking data on EPL, used as an instrument for the employment rate in equations for women. }\end{array}$}} \\
\hline & & & & \\
\hline \multicolumn{5}{|c|}{ Source: European Labour Force Survey; US Current Population Survey; Bassanini and Duval (2006); OECD Taxing Wages; } \\
\hline Conway, De Rosa, Nicoletti and Steiner (2006 & & & & \\
\hline
\end{tabular}

80. In this context, it is important, for interpretation purposes, to analyse the advantages and limitations attached to each of the estimations:

- The first estimation is based on a larger sample of countries and exhibits wider dispersion in terms of both hours worked and taxation. However, the taxation variables suffer from measurement error because assumptions have to be made in the aggregation procedure in order to map the tax indicators - differentiated by the presence of children - into working hours data from labour force surveys.

- The second estimation is closer to the microeconomic approach because the data are defined at a more disaggregated level. The estimation is more behavioural in that respect. Measurement error 
in the taxation variables is reduced, as the tax indicators have an almost direct mapping into the population groups defined by the labour force surveys.

81. As mentioned above, the focus is on the estimated $\delta$, proxying for a semi-elasticity of hours worked, conditional on employment, with respect to marginal taxation. Estimates of the baseline model confirm the higher responsiveness of women to taxation. Indeed, the estimated parameter is approximately seven times higher for women than for men in the sample without children. This parameter is statistically negative and significant for both men and women in this larger sample. It is, however, extremely low and even insignificant for men in the sample in which it is possible to control for the presence of children. This confirms the importance of the disincentive effects attached to marginal taxation for women, who are most often second earners.

82. For women, the tax-rate elasticity is robustly estimated to be of the same order of magnitude across samples, between -0.7 and -0.8 . This estimate is quantitatively quite small: a decrease in the marginal tax wedge of one percentage point, everything else being equal, implies a $0.7-0.8 \%$ increase in hours worked, conditional on employment. Although not precisely comparable with the literature for the reasons outlined above, this estimate is slightly lower than average findings. ${ }^{46}$ This is consistent with the conjecture that, contrary to most of the estimates found in the literature, and in particular in the crosscountry literature, the empirical approach undertaken here is intended to disentangle the intensive margin from the extensive margin, and conversely to focus on responses at the intensive margin of labour supply.

83. The impact of the average tax wedge is negative and significant in the first sample and positive, although not significant, in the sample in which it is possible to control for the presence of children. The interpretation given here is as follows. In the second sample, the estimation can be considered closer to the microeconomic approach, and might actually capture an expected positive income effect, by reducing measurement error in the taxation variables. This interpretation is consistent with the empirical finding that estimating the specifications presented in the first two columns of the above table (i.e. without children) on a restricted sample - covering the countries for which the data on the presence of children are available - does not substantially alter the results. This suggests that it is not the sample size per se, but rather the aggregation procedure, that might induce measurement error as well as increase the influence of potential multicollinearity problems among the marginal and average tax wedges. ${ }^{47}$

84. The interactions between the average tax wedge and the categorical variables (education level, marital status) are estimated to be statistically similar across the samples. The interactions are only significant in the equations for men, due to higher precision of the estimates. The sign of these interactions - negative for high-educated individuals and married individuals - can be interpreted as evidence of negative income effects, as suggested above, although this is difficult to assess in the empirical framework developed here. In both samples, the sign of the estimated parameters on the interaction between marital status and the average tax wedge is reversed across sexes, suggesting the possibility of joint labour supply decisions and household division of work time. Unfortunately it is not possible to go further in that

46. In terms of the Prescott model - in which the wage elasticity and the tax-rate elasticity are linked by the following equation: $\frac{\delta \ln \boldsymbol{h}}{\delta \ln w}=-(1-t) \frac{\delta \ln \boldsymbol{h}}{\delta t}$ - this elasticity compares with an implied tax-rate elasticity of -1.5 , hence a wage elasticity of 0.75 , while the estimate presented here implies a wage elasticity of approximately 0.3 - 0.4 (assuming a 50\% marginal tax wedge).

47. However, multicollinearity should not be a serious concern here: taxation variables are measured in levels, because of the use of a within-estimator across countries and groups. Hence, the residual correlation among the two variables is essentially within countries and groups across time. Multicollinearity tests suggest that the issue is not severe here. 
direction, the data being semi-aggregated and therefore precluding identifying spouses in the sample. ${ }^{48}$ The interaction between the average tax wedge and the presence of children is negative both for women and men, although not significant. This result contradicts previous findings from the literature of important income effects for women with children (see Blundell et al. 1998), although one possible interpretation is that the income effect mostly occurs through the participation decision.

85. The impact of the employment rate is insignificant, at this level of disaggregation, suggesting the negative significant effect found at the aggregate level mostly reflects compositional effects arising through women's participation and employment. This intuition is confirmed by the negative sign estimated on the corresponding parameter in women' equations - though it is statistically insignificant-, suggesting higher employment rates for women have corresponded to a higher proportion of part-time female employment.

86. The following analysis on working-time regulations and other labour and product market policies makes use of the sample where it is not possible to identify the presence of children, due to the narrow country coverage of the alternative, more disaggregated, sample. Despite the differences that have emerged for the impact of the average tax wedge across the two samples, the interpretations which have been discussed before, it is important to signal that all of the following results on institutional variables are robust to the change of the sample size.

\subsubsection{Working-time regulations matter! (for men...)}

87. The literature on the impact of working-time regulations has focused on the consequences, within countries, of domestic reforms in the relevant legislation. ${ }^{49}$ One important finding is that the effect of regulations on employees' working time schedules are extremely heterogeneous - and even opposite across genders, occupations, firm sizes, and sectors (see Skuterud, 2007, for a convincing naturalexperience estimation in Québec; Afsa and Biscourp, 2004, on France). Figure 3 displays a cross-country scatterplot of weekly hours worked versus weekly hours regulation, for men and women respectively. This scatterplot indicates the heterogeneous impact of working-time regulations. Indeed, whereas there seems to be a positive relationship between the regulatory indicator of weekly hours worked (a higher value implying less restriction) and actual hours worked for male employees, this relationship does not hold for female employees. ${ }^{50}$ The following empirical analysis investigates this topic more formally.

48. An attempt was made to estimate the model by three-stage least squares, thus taking into account correlation among male and female labour supply equations; the results for the elasticity of hours with respect to taxation were substantially unchanged. This procedure is, however, discarded here for technical reasons.

49. The most cited examples are Hunt (1999) for Germany, and Crépon and Kramarz (2002) for France. For a complete assessment, see Cahuc et al. (2006).

50. Note that the impact is even reversed for women when excluding the United States from the scatterplot. 
Figure 3. Weekly hours worked among employees and working time regulations

\section{A. Men}

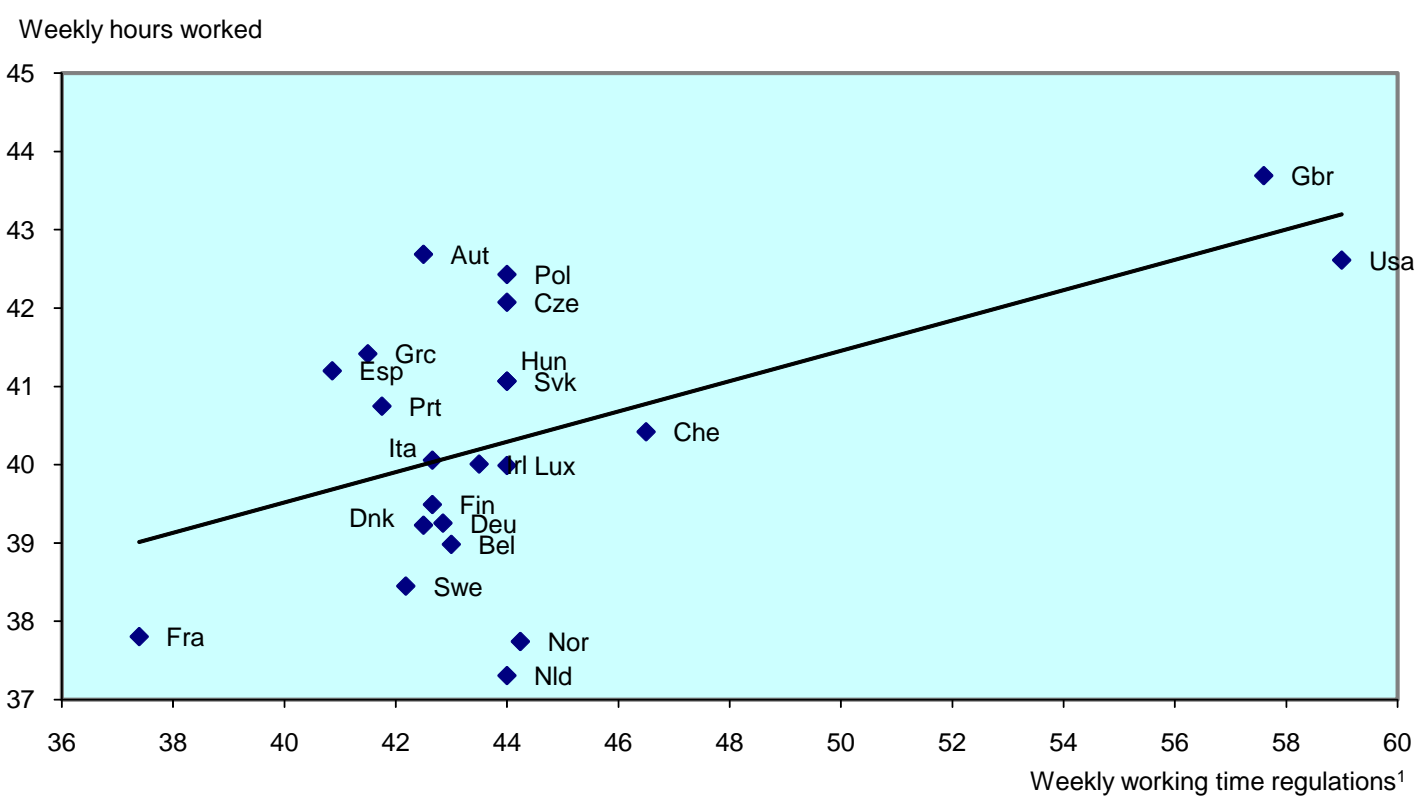

B. Women

Weekly hours worked

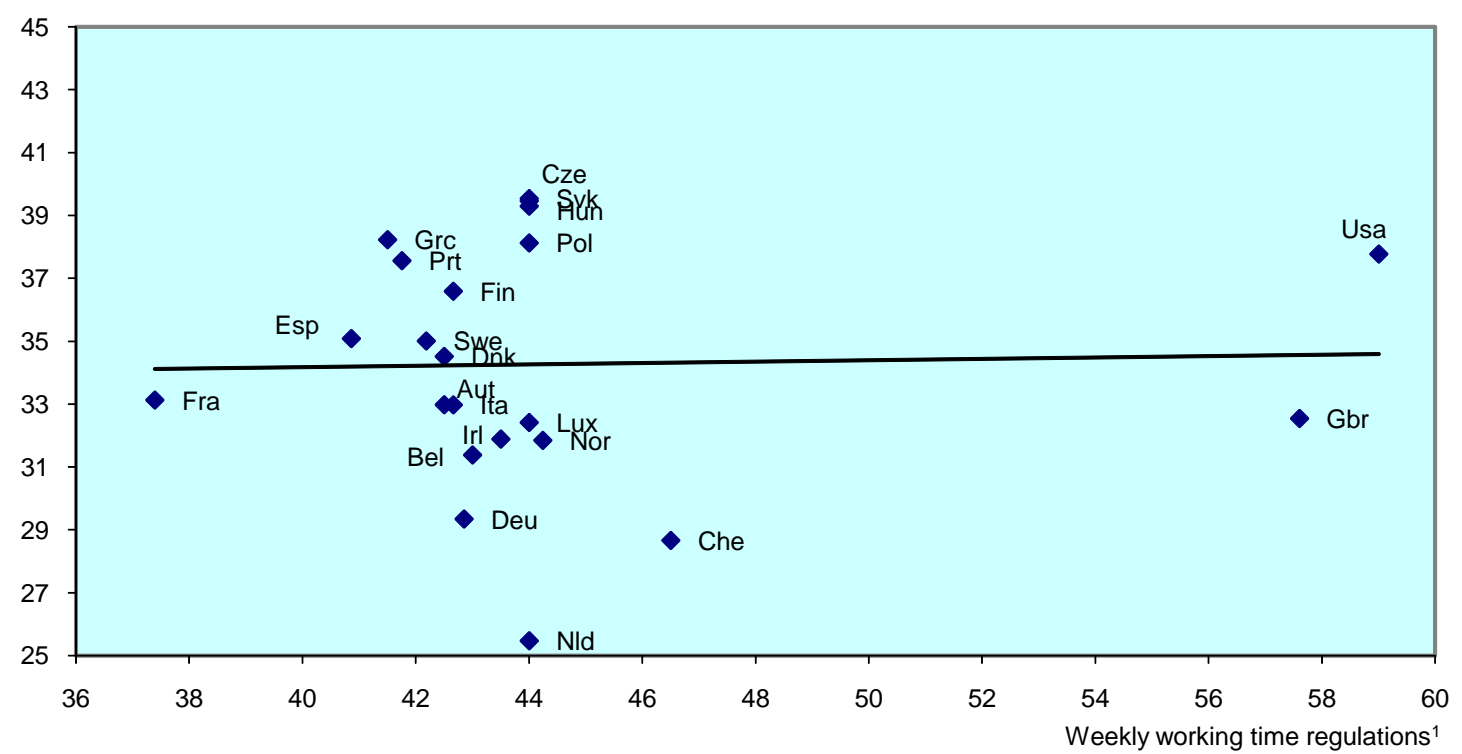

Notes: Prime-age labour force (25-54). Data refer to 2005.

1. Average of weekly normal hours and of weekly normal hours and overtime.

Source: European Labor Force Survey; US Current Population Survey and OECD calculations. 
88. The estimates presented here are built on the baseline specification, as presented in the last section. The policy variable is introduced both in levels and interacted with education levels. ${ }^{51}$ The indicators used refer to standard normal weekly hours, and an average of regulated normal plus maximum overtime hours. Two results emerge from these estimates (Table 6):

- Flexible working-time regulations are found to have a significant positive impact on men, and a slightly significant impact on women. This result is in line with the literature (see Skuterud, 2007). An important part of the explanation is that women, in particular low-skilled women, are likely to have weaker attachment to the labour market, and, importantly, work fewer hours than men, so that the impact of maximum workweek regulations is less pronounced for them. ${ }^{52}$ This is consistent with the finding that the significant impact of working-time regulations is found only in the case of high-skilled women, who have stronger attachment to the labour market and typically work longer hours than their low-skilled counterparts.

- The impact of working-time regulations governing weekly normal hours decreases with education levels, suggesting that limitations are not binding for workers at the high end of the earnings / education distribution (for example managers and academics), but are important for low and medium-educated employees. The negative impact on high-skilled workers implies that tighter working hours regulation increase actual hours worked for certain managing occupations, possibly because they have to "compensate" for the lower working-hours of workers for whom the legislation is binding. Indeed, country-specific studies show that working-time reductions increase the impact of irregular working hours for managers and/or in big firms (see Afsa and Biscourp, 2004). ${ }^{53}$ This is also suggested by the use of a synthetic indicator, covering both weekly normal hours and overtime. Indeed, in this case, the results indicate a positive effect on actual hours worked by men, increasing in the education level. ${ }^{54}$

- Finally, the third column of Table 6 exhibits a negative significant coefficient on the employment rate in influencing hours worked by men - contrary to the other specifications - when working-time regulations are controlled for. Though the significance level is weak, this result could suggest some "work sharing" effects of working-time arrangements. ${ }^{55}$

51. While the literature would also suggest that the impact differs across firms and sectors, this information is not available here.

52. The results are also consistent with the intuition based on an insider/outsider setting, in which, in unionised and regulated markets, insiders are able to bargain for shorter work time, eventually at the cost of higher unemployment for outsiders. This intuition is confirmed in the first stage of the present regression, although the framework does not allow formally testing assumptions on the impact of a working-time regulations reform. See Cahuc et al. (2006), for a model of the impact of workweek reduction in a unionised environment.

53. This result is also consistent with a model in which, when the hourly wage is taken as given, reductions in standard hours are expected to increase working hours by causing the number of overtime hours to rise. This occurs if the level of standard hours is low relatively to what the firm needs. In this case, a reduction in standard working-time has the effect of increasing actual hours worked and reducing employment (see Calmfors and Hoel (1988), Cahuc et al. (2006) and Skuterud (2007)).

54. This finding does not apply to the estimates for women, for whom it is clear that the negative impact found on high skilled employees is just a reflection of the normal weekly hours impact, as presented in the second column. Indeed, the synthetic indicator gives a higher weight to normal hours regulation than to overtime regulation, since it is defined as the average between normal hours and the average of normal hours and overtime. Furthermore, none of the estimated parameters on working time regulations is statistically significant in column 4 .

55. This finding has to be taken with care. It is contradicted by the use of a simple normal hours indicator in column one, because the latter indicator should, a fortiori, deliver a similar result. 


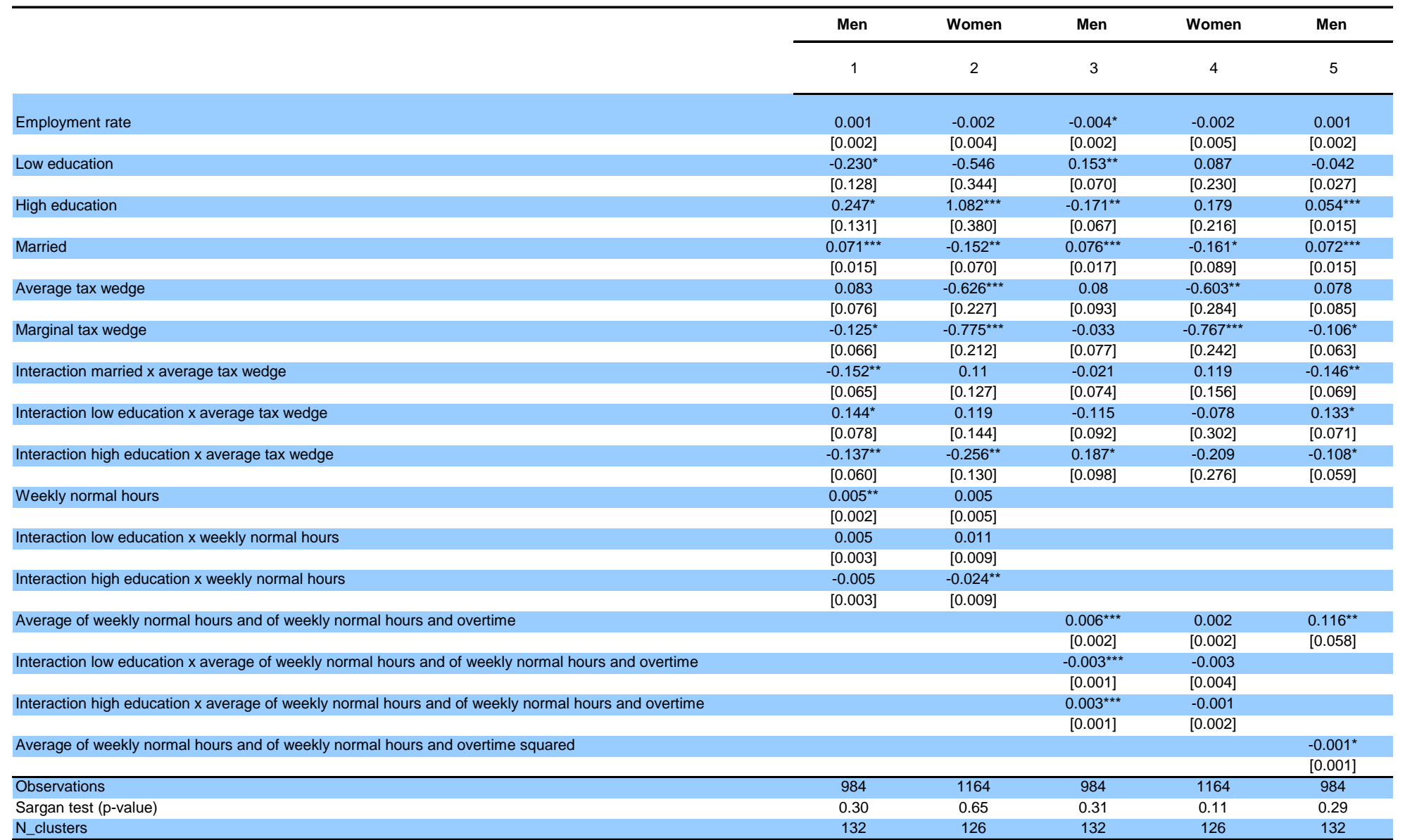

Notes: ${ }^{*} p<.1,{ }^{* *} p<.05,{ }^{* * *} p<.01$. Each equation includes country and year dummies.

Weighted estimation. Standard errors in brackets are clustered by country $\mathrm{x}$ marital status $\mathrm{x}$ education. The employment rate is instrumented as follows:

Equations for men: Instruments are marital status interacted with education level and lagged average replacement rate interacted with education level. Equations for women: Instruments are marital status interacted with education level and lagged EPL on regular contracts interacted with education level.

The countries included in the panel are: Austria, Belgium, Czech Republic, Denmark, Finland, France, Germany, Greece, Hungary, Ireland, Italy, Luxembourg, the Netherlands, Norway, Poland, Portugal, Slovakia, Spain, Sweden, Switzerland, the United Kingdom and the United States. Differences in the number of observations between estimates for men and women are due

to the absence of Luxembourg in equations for men. This absence is caused by lacking data on EPL on regular contracts, used as instrument for the employment rate in equations for women Sources: European Labour Force Survey; Current Population Survey; Bassanini and Duval (2006); OECD Taxing Wages, Conway; De Rosa, Nicoletti and Steiner (2006) and OECD calculations. 
89. While working time regulations are found to influence actual hours worked for men in general, their impact is likely to be particularly strong in countries with relatively strict regulations. In countries where normal hours are relatively long, actual hours worked will not be constrained, as few workers will be directly affected. However, when normal hours are relatively short, the statutory length of the work week is likely to be much more binding. This is confirmed by econometric analysis. The results of the last column of Table 6 show that weekly hours working-time regulations have a non-linear impact on hours worked. ${ }^{56}$ This suggests that the binding effect of regulation is stronger in countries where the ceiling on hours is comparatively low. ${ }^{57}$

90. Finally, is important to note that the introduction of working-time regulations reduces the impact of the marginal tax wedge on working hours of men to statistical insignificance, supporting the view of labour supply of men being unresponsive to taxes, at least for the range of earnings considered in the present analysis. $^{58}$

\subsubsection{Other labour and product market policies also matter...}

91. This section investigates the impact of labour and product market policies on hours worked of different groups in the labour force, conditional on employment. While the literature has produced an important amount of research on the relationship between taxes and hours worked, it has been rather silent on the impact of other labour market policies, as mentioned earlier. The exception to this pattern is the debate on the role of unionisation to understand cross-country evolution of hours worked.

92. The estimates presented here are built on the same specification as those presented in the previous section. The policy variable is introduced both in levels and in interaction with the education of the employee, per each gender. The identification of an heterogeneous impact of institutional settings on labour market outcomes, depending on workers' characteristics, is a recent feature of the cross-country literature, mostly based on the heterogeneity of labour supply parameters, but also on its interactions with labour demand considerations. The estimates presented here (Table 7) show in turn the separate impacts of EPL on regular contracts, product market regulation and union density. ${ }^{59}$

56. Only regressions for men are presented, due to the very low statistical significance found in estimates for women. The interaction with the education level is not taken into account because it is not relevant to the assumption tested here while potentially introducing multicollinearity in the estimation.

57. Some caution is, however, needed when extrapolating these results. Indeed, the impact of working-time regulations is identified though policy changes within countries over the sample period (1996-2005) - by definition of a within country estimator. Hence, the estimates are likely to depend on the limited number of countries having undertaken significant reforms over the decade (the most important reform being the French 35-hours week).

58. As mentioned above, marginal taxation at very high earnings might have a disincentive effect on labour supply of men, an effect that cannot be captured by the aggregation procedure used in this work to compute average marginal tax wedges.

59 Structural policies generally exhibit little time variance and strong multi-collinearity with each other. This is why policies are mostly considered here one at a time. There is no straightforward way to correct for multi-collinearity. Looking at policies separately prevents the influence of one policy from being blurred by collinearity with another. However, as long as hours worked are jointly influenced by several policies, analysing them separately generates a problem of omitted variables. This implies that the results must be interpreted with care. 
Table 7. Econometric estimates - Product and labour market policies

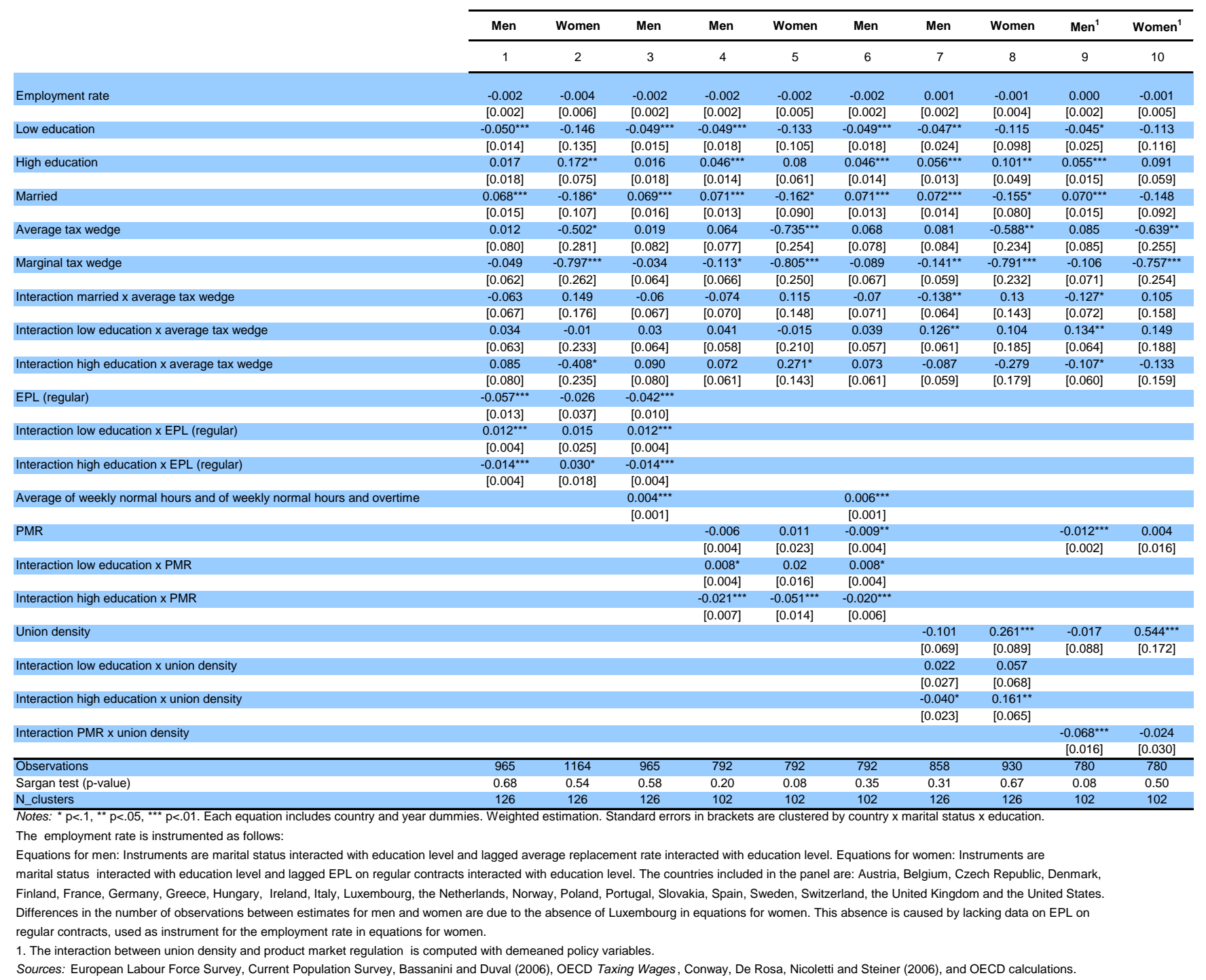


93. The results show that the strictness of EPL for workers on regular contracts has a negative impact on hours worked by men, and that this impact increases with the level of education. The impact on women's hours worked, conditional on employment, is insignificant (columns 1 and 2). These findings can be interpreted in different ways:

- This suggests that protected workers in regulated markets can benefit from lower working hours, eventually at the cost of raising unemployment for marginal workers. This is supported by the interaction of EPL with the education level in the regression analysis, which is positive and significant for low-skilled men and negative for high-skilled men.

- This result is also consistent with findings in Bassanini and Duval (2006), who argue that the stringency of EPL may encourage the use of part-time work so as to circumvent the legislation affecting full-time jobs and show that high EPL on regular contracts is associated with a substitution of part-time for full-time work for female. ${ }^{60}$

- Another interpretation in line with this finding is that in countries with low EPL, higher hours worked might be a signal of greater work effort in an asymmetric information setting. ${ }^{61}$

- This finding is in line with the predictions of the Michelacci and Pijoan-Mas (2007) labour market search model, in which labour market tightness increases the incentives to work longer hours by increasing the intertemporal return to hours worked.

It is important to note that the result is, however, far from being unambiguous from a theoretical perspective. In a model in which the intensive and extensive margins are substitutes, any policy that acts directly on one of these margins is likely to lead to adjustments costs on the two margins going in opposite directions. In this context, firing costs have a direct effect of making it more costly to use the extensive margin and as a result lead to opposing effects on the intensive margin. ${ }^{62}$

94. Finally, as for working-time regulations, introducing an indicator of EPL on regular contracts makes the impact of marginal taxation on men's working hours insignificant, reinforcing the argument that while taxes have an important impact of women's working hours, working-time regulations and employment protection legislation have greater effects on hours of work supplied by men.

95. The impact of the stringency of product market regulation is similar to the one found for EPL (columns 4 and 5). Product market regulation has a negative impact on working hours for men, conditional on employment, and this impact is strongest at high levels of education. Among women, only hours worked by high-educated women are affected, negatively, as men, by the stringency of product market regulation. This result can arise from a sectoral effect, whereby workers in protected sectors can benefit from the monopoly power of firms by negotiating lower working hours. It is also consistent with other more specific models:

- In a simple right-to-manage bargaining model, such as that presented in Cahuc et al. (2006), it can be shown that the negotiated number of hours (between the trade union and the firm) is a

60. Although not significant, the negative effect found in the equations for women, decreasing with the education level, is suggestive of this interpretation, insofar as the over-representation of women in parttime jobs is probably lower for highly skilled workers.

61. This is the interpretation given by Faggio and Nickell (2007) to a similar finding, although the authors use aggregate data on average hours worked.

62. See Fang and Rogerson (2007). 
function of a number of institutional features, among which the elasticity of the revenue function of the firm with respect to the services of labour (positive impact). This elasticity can reflect the monopsony power of the firm in the labour market and is low when the firm has strong market power. This implies that economies with less competition in the product market should have lower working hours.

- If regulations take the form of nominal payments and these payments are rebated to households, as modelled in Fang and Rogerson (2007), then higher regulation is associated with less time devoted to market work. This is simply a negative income effect. The key driving force is the size of the implied transfer payments relative to total labour income.

96. Finally, columns 3 and 6 show that the impact of EPL and product market regulation on working hours of men are robust to the inclusion of working-time regulations, confirming the idea that these policies operate through distinct channels on labour market outcomes among OECD countries.

97. Unionisation, measured by union density, is the last institutional setting that is analysed in this framework and is not new to the literature. ${ }^{63}$ Estimates suggest a significant and opposite effect on hours of work of men and women, conditional on employment: while high union density is associated with lower working hours for men, it is associated with higher working hours for women. ${ }^{64}$ These results support findings reported in the literature on the role of unions in the bargaining process:

- Alesina et al. (2005), as mentioned before, show that unions can restrict labour supply in order to increase wages. This effect can be also obtained through a simple right-to-manage model in which the union can bargain a higher utility level for its members (see Cahuc et al. 2006). Insofar as men are most likely to have a heavier weight in unions' objective function, this prediction is consistent with the estimates of hours of work supplied by men.

- Trade unions have generally fought against part-time work, seen as a threat to full-time standards (see Houseman (2001) for econometric evidence). To the extent that women are over-represented in part-time jobs, the results of estimates of women hours of work is consistent with the idea that high union density reduces the proportion of part-time jobs and therefore raises average hours worked by employed women.

- These results are also consistent with the finding in Bassanini and Duval (2006) of a positive and significant effect of union density on female full-time employment and a negative impact on parttime work, suggesting unions' objectives to promote full-time as opposed to part-time opportunities.

98. Differences between estimates for men and women in the impact of unionisation are notable. Indeed, while the negative impact of union density on working hours for men is weak and confined to high skilled workers, the positive impact found on working hours of women is high and significant. This suggests that the role of trade unions in shaping differences in weekly hours worked among OECD countries mostly occurs through its influence on the proportion of part-time work in the labour force.

63. Note that while union coverage, i.e. the share of employees covered by collective contracts, would have been a better measure for the purpose of this work, it is not possible to use this variable in a panel context because data are not available on a time-series basis.

64. Note that in the case of men, the impact is significant only for high levels of skills. 
99. The analysis can be extended to study potential interactions among unionisation and product market regulation. As column 9 shows, the negative impact of union density on working hours of men increases in the stringency of product market regulation. This result is consistent with recent literature findings. A number of studies have investigated the impact of product market deregulation across different labour market settings. ${ }^{65}$ Fiori et al. (2007) show that employment gains from product market deregulation are largest in situations in which labour market settings provide strong bargaining power to workers. When both product market regulation and unions' bargaining power are high, workers - in particular insiders can extract a higher proportion of the rents generated by market power. In this context, the negative correlation between unionisation and hours worked by employed individuals is expected to increase with product market regulation. This result is confirmed in the present analysis for working hours of men; by contrast, the same interaction is estimated to be insignificant in women' estimates (column 10), consistent with the different role of unions in the shaping of women's working patterns. This finding, associated with the weaker direct estimated impact of unions on working hours of men than of women, suggests that the level of product market regulation is a important channel through which unions allow insiders to benefit from market power.

\subsection{Country-fixed effects and omitted variables}

\subsubsection{The measure of our ignorance: the importance of country-fixed effects}

100. The empirical estimates presented in this work suggest that taxation, working-time regulations, and labour and product market institutions are significant explanatory variables of differences in hours worked across countries and over time. It seems, however, that policies, at least as they are measured here, are not sufficient to fully account for observed differences in hours worked. Indeed, country-fixed effects included in the model play a non-negligible role in the estimation. These effects can be dubbed as the measure of ignorance. One way of presenting the explanatory power of country-fixed effects is by simulating the model while setting the country-fixed effects to zero, and compare the simulated working hours with that predicted by the model. This is done below for men and women separately, and is shown graphically in Figure $4 .{ }^{66}$ The reference country is the United States.

101. These results can be summarised as follows:

- The importance of country-fixed effects in the model has a gender-specific pattern. The country-fixed effects in the regression for women are statistically significant only in the case of three countries (France, United Kingdom and Ireland) whereas they are statistically significant for slightly more than half of the countries in the case of men. However, the explanatory power

65. See Nicoletti and Scarpetta (2005), Griffith et al.(2006), Amable et al. (2006) and Fiori et al. (2007).

66. For the purpose of this simulation, country-fixed effects are first re-estimated in a specification that includes taxes, working-time regulations (using the indicator covering normal and overtime hours) and other labour and product market regulations. In so doing, they correspond to a residual component that remains unexplained by other institutional variables. The sample is reduced in this case because of lack of coverage for some countries on product market regulation data. These country-fixed effects are set equal to zero and weekly hours worked are recalculated using this modified equation. The impacts reported in the figure are calculated by making the difference between the predicted data on weekly hours worked and weekly hours worked estimated with the modified equations where country-fixed effects are set to zero. This difference corresponds to the contribution of the country-fixed effect in the empirical model. In this way, a positive impact in Figure 4 corresponds to a positive country-fixed effect. 
of country-fixed effects within the model is much higher in the case of women than in the case of men.

- While country-fixed effects are always found to be positive for men, their sign varies for women. Marginal taxation, even in countries where it is comparatively high, such as Northern European countries, does not appear as a sufficient explanation of working hours differences for women. While major discrepancies occur in the case of the Netherlands and Switzerland, the case of Ireland and the United Kingdom is also interesting. Indeed, despite low levels of marginal taxation, women's average working hours in Ireland and the United Kingdom are very low compared with other OECD countries. At the same time, working-time regulations do not seem either to fully explain men's hours worked. France is a good example where estimates for both men and women display significant positive country-fixed effects, despite high levels of marginal taxation and restrictive working-time regulations.

What factors could stand behind the country-fixed effects? One obvious hypothesis is that of omitted variables. Due to data limitations, the model presented here lacks two important dimensions in this respect, namely childcare-related costs and benefits, as well as other social benefits excluded from the indicators used in the present analysis. The potential role of these factors is outlined below. ${ }^{67}$

67. Another omitted variable that could be driving part of the country-fixed effects is the presence of regulations affecting part-time work other than that embedded in taxation systems. Hence, the negative country fixed effect in the Netherlands may reflect the existence of regulations favouring part-time work that are not taken into account in the rudimentary index of working-time regulations used in the estimates. In the same vein, the United Kingdom presents some form of exemptions from the national insurance payment for workers working less than 16-hours week, mainly mothers. Also, another potential omitted variable pertains to industrial structure differences across countries. Indeed, cross-country differences in the sectoral allocation of the labour force are non-negligible in OECD countries. At the same time, there are systematic differences in working hours across industries. The simulation presented here does not include, due to data limitations on institutional variables, the countries that are most likely to differ in terms of labour allocation, notably Eastern European countries. Unreported country-fixed effects, when these countries are included in the sample, suggest that some of them, notably the Czech Republic and Hungary, exhibit important positive fixed effects, in particular for men, in concomitance with a still important proportion of the labour force in the agricultural sector, where hours of work tend to be particularly long. 
Figure 4. Hours impact of country fixed effects, men and women

Per cent difference vis-à-vis the United States

A. Men

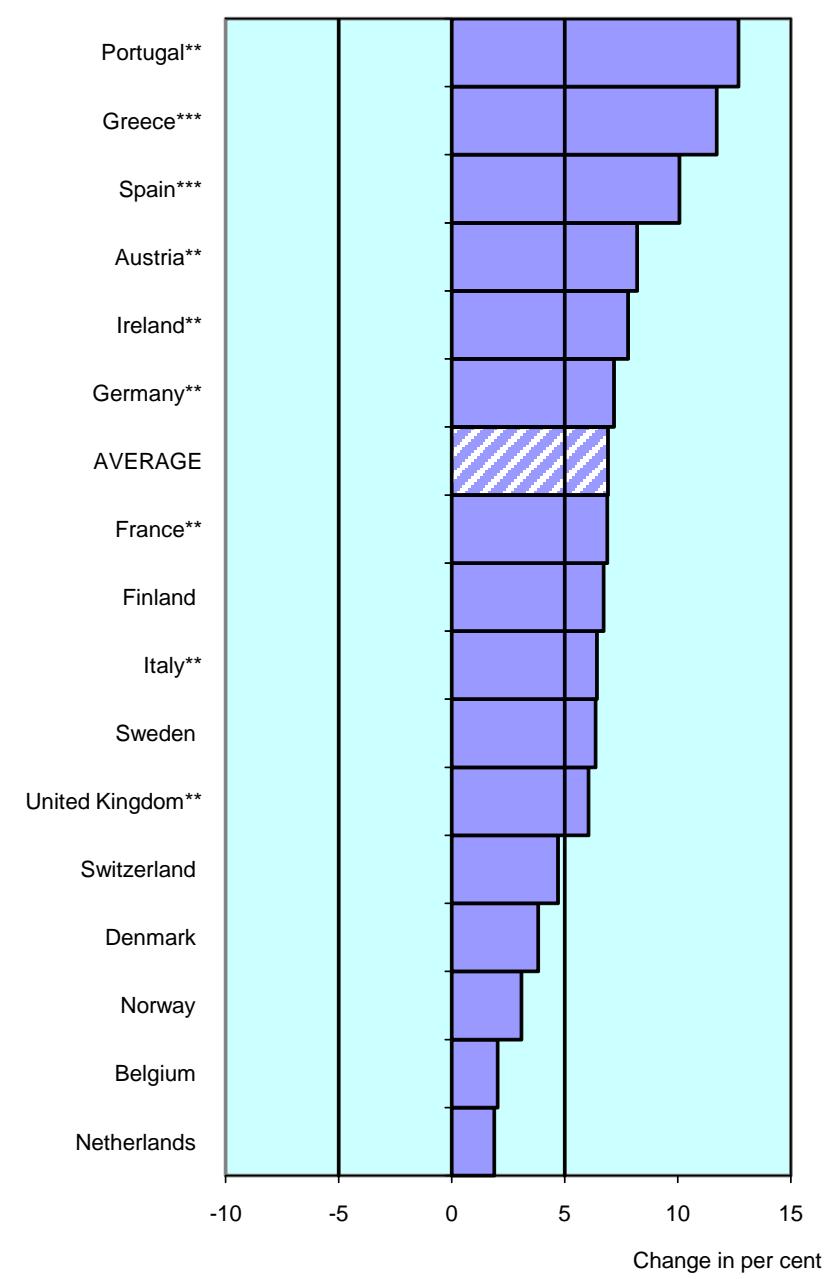

B. Women

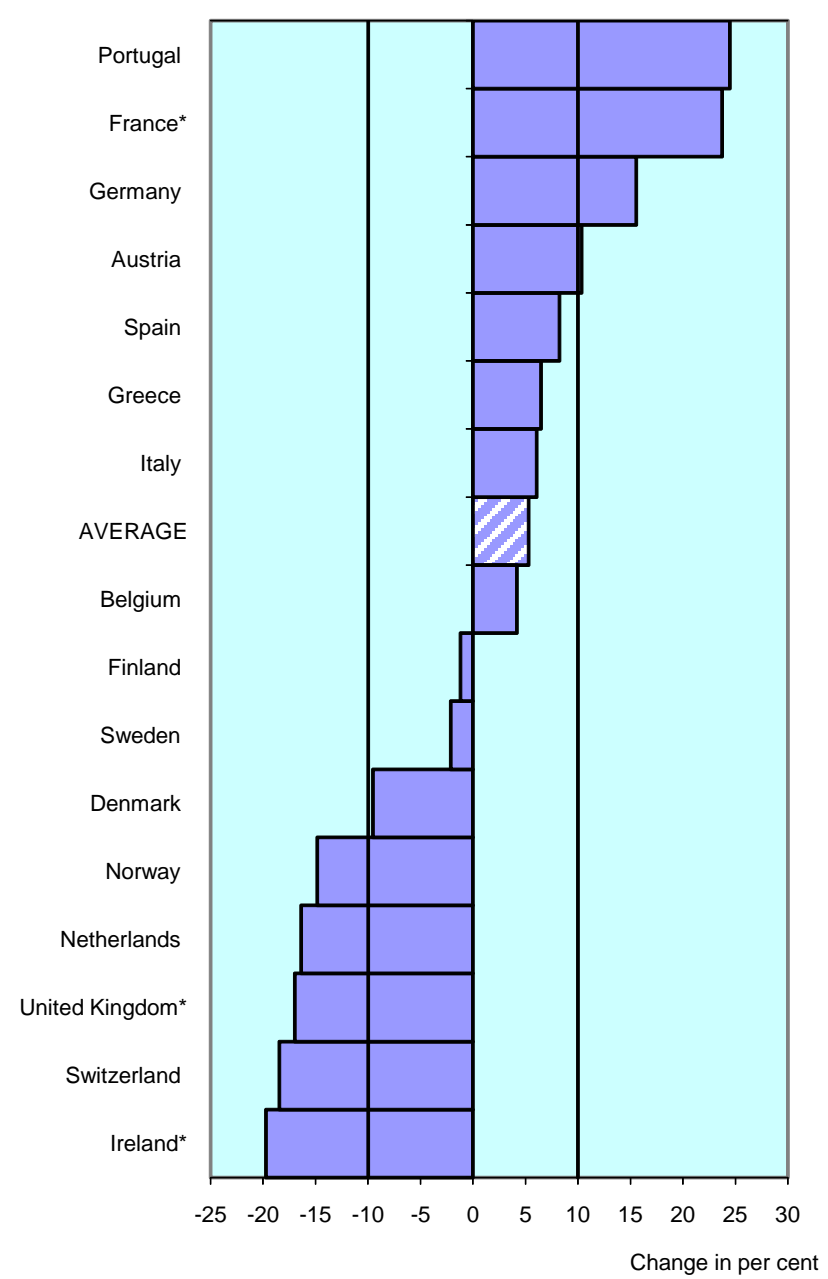

Notes: Statistical significance of the country fixed effects: ${ }^{*} p<.1,{ }^{\star *} p<.05,{ }^{* \star} p<.01$. Data refer to 2002 , average for OECD is weighted by employment levels.

Source: OECD calculations (see text).

\subsubsection{Omitted variables: Childcare costs and benefits}

102. The model presented in this study includes in the taxation indicators the tax reliefs and family cash transfers universally paid in respect of dependent children between five and twelve years of age who are attending school. However, this information is only partial and does not allow uncovering the very different institutional setups or welfare state regimes shaping financial work incentives for parents. In particular, it is not possible to incorporate in the estimation the fees charged by childcare centres, childcare benefits, as well as other child-related benefits. 
103. Comprehensive work by Immervoll and Barber (2005) has shown that there can be important adverse work incentives for parents in situations where childcare costs are high. In this respect, it is suggestive to note that the authors cite Switzerland and Ireland as two countries where high childcare costs create adverse work incentives for parents. However, while the impact of childcare on employment incentives has been established to be important, little is known about the relative incentives on the intensive margin of labour supply. ${ }^{68,69}$

68. An exception is Geyner and Steiner (2007), who estimate the short-run and long-term effects of childbirth on married women's employment and working hours in four European countries with different institutional regimes (Denmark, Germany, Italy and United Kingdom). The authors find that compared with the other countries, mothers in the United Kingdom and Germany reduce their working hours substantially both in the short- and in the long-run. The authors interpret this finding in light of the distinct country-specific institutional arrangements governing childcare. In Germany, rationing of publicly subsidised childcare facilities for children below the age of three causes negative short-run effects of childbirth on women employment levels; relatively flexible work schedules enable mothers' long-term adjustment in employment levels via part-time work. In the United Kingdom, a similar outcome arises as a result of a different institutional regime: the absence of subsidised childcare might force mothers to work part-time to reconcile childcare responsibilities and employment, which is supported by in-work benefits for households with children (Haan and Myck, 2006), and flexible working-time arrangements. The situation is different in Italy, where there is no adjustment in hours of work after childbirth, because the institutions do not allow flexible working-time arrangements so that women mostly have to decide whether to work full-time or to completely withdraw from the labour market in order to look after their young children (Del Boca, 2002). Recent research on Australia reports contrasting results: Rammohan and Whelan (2007) find that childcare costs have a statistically insignificant effect on the decision to work either full-time or parttime for married women, suggesting that a policy committed to increasing the amount of financial support available to users of childcare may have a limited effect in increasing the labour market activity of married mothers. However, these authors only consider the cost of childcare, which is probably only one of the elements of an overall institutional framework surrounding childcare policies.

69. In particular, while some countries encourage flexible working-time arrangements, reflected in high incidence of part-time work among mothers, it is not clear whether a movement towards shorter hours is good or bad for women. On the one hand, it may reflect a desire on the part of women to work less to allow more time for family care. On the other hand, part-time work often pays less well and is argued to have a lower status and less career prospects than full-time work. Part-time work can reflect a constrained choice due to the absence of subsidised childcare, as seems the case in the United Kingdom, in particular for lowincome families (see Geyner and Steiner, 2007). The evidence is mixed, though. In a recent empirical work on job satisfaction and family happiness in the United Kingdom, Booth and van Ours (2007) highlight what they call the "part-time puzzle": while hours satisfaction and job satisfaction indicate that women prefer part-time jobs, life satisfaction is not affected by hours of work. 
Figure 5. Childcare costs ${ }^{1}$ and country-fixed effects

\section{A. Women}

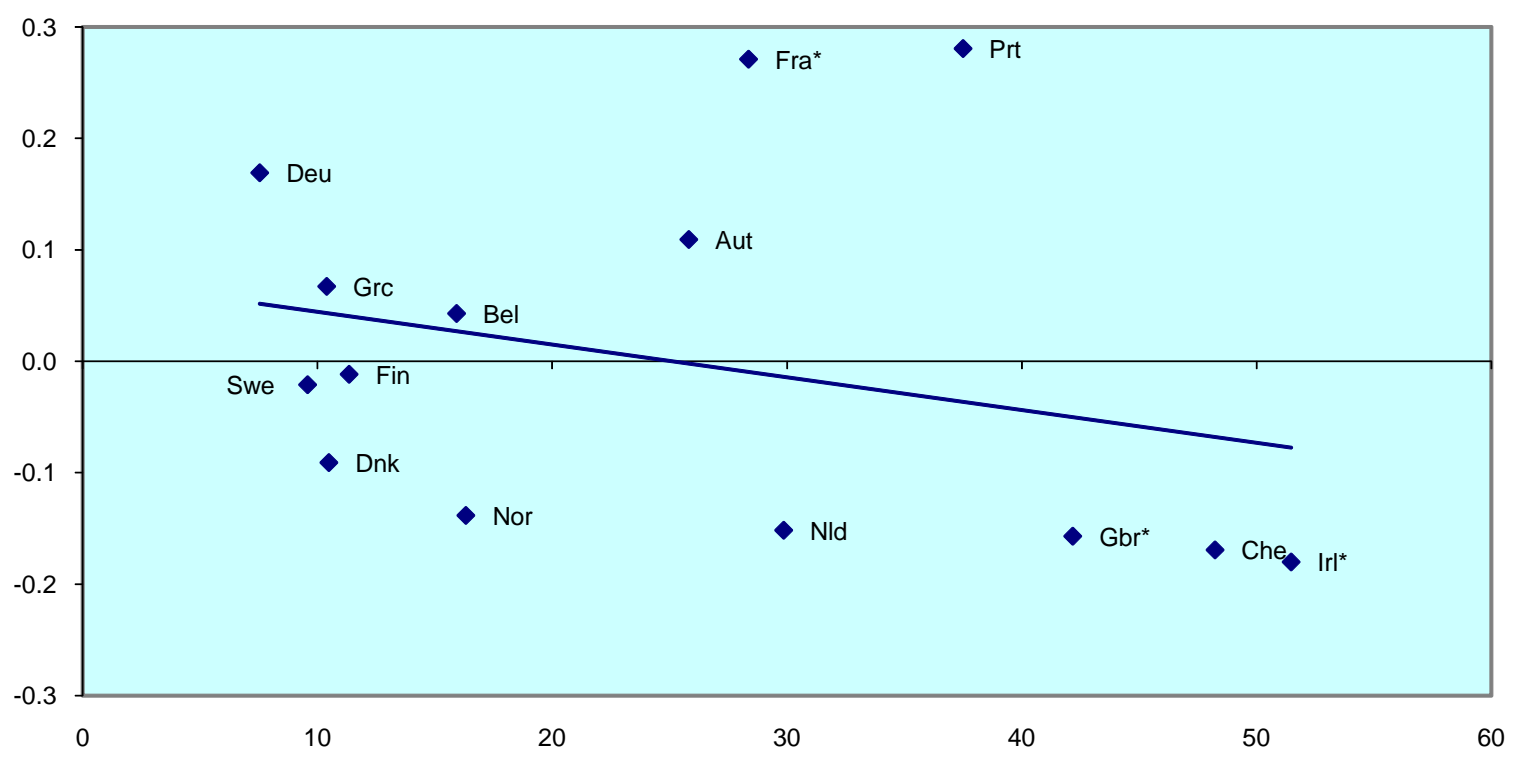

B. Men

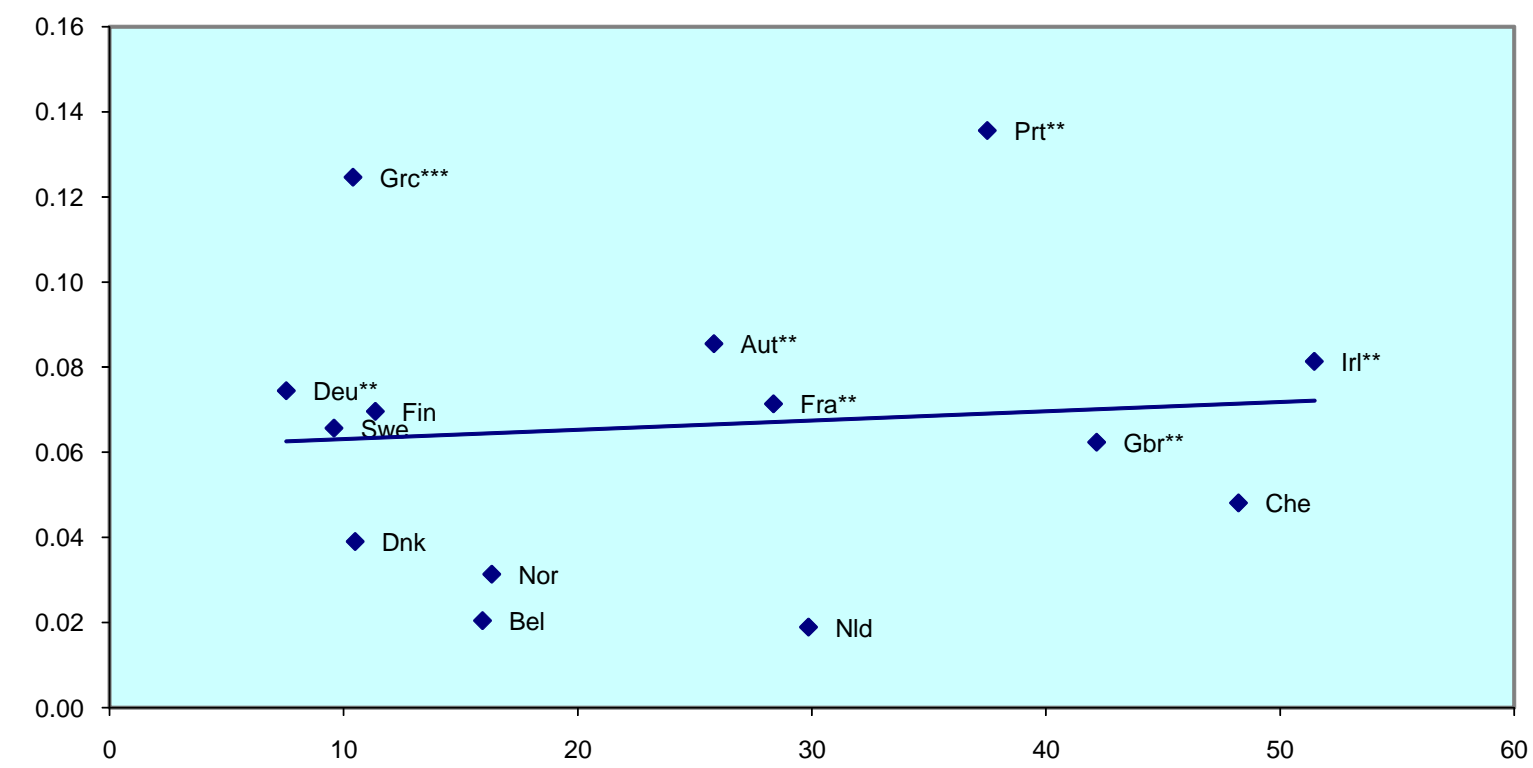

Notes: Statistical significance of the country fixed effects: ${ }^{*} p<.1,{ }^{* *} p<.05,{ }^{* * *} p<.01$. Data refer to 2002.

1. Costs are reported for a two earner couple with two children with full-time earnings at $200(100+100)$ per cent of APW. Some countries are missing to due missing data on childcare costs.

Source: Immervoll and Baber (2005). 
104. Figure 5 provides an illustrative evidence of the potential for childcare costs to partly explain variation of the country-fixed effects. Total child-related costs are negatively associated with country-fixed effects in the regressions for women (Panel A), while they are slightly positively associated with country-fixed effects in the regression for men (Panel B). This opposite gender-specific pattern suggests the existence of family labour supply decisions, or household allocation of work, as outlined above. Investigating the potential positive and negative effects of childcare costs on labour supply decisions is an important issue for future research.

\subsubsection{Omitted variables: Income-tested or hours-dependent benefits}

105. A combination of tax increases and benefit withdrawal can reduce the financial incentives for increasing work effort. Marginal effective tax rates can be used to measure these disincentives, but it is not possible to use these data in the empirical analysis presented here. ${ }^{70}$ While the tax indicators used in this work incorporate a number of in-work benefits, notably those that take the form of non-wastable tax credits (for example the Working Families Tax Credit in the United Kingdom), other benefits, potentially interfering with hours of work, are excluded. This is the case of housing benefits, family benefits, or social assistance. While the withdrawal of some of these benefits, in conjunction with higher taxes, might make additional work not-rewarding in the short run, these disincentives effects are likely to be of second-order compared to those already incorporated in this analysis (notably in-work benefits), at least for the range of earnings considered here. ${ }^{71}$ Figure 6 plots country-fixed effects from the regression for women against a measure of the so-called "low-wage trap", namely average effective tax rates — hence including both tax increases and benefit withdrawals - associated to the transition form part-time to full-time work. ${ }^{72}$ As can be seen from this figure, there is a slightly significant negative association between country-fixed effects (in the regression for women) and marginal effective tax rates on part-time employees. This suggests that part of the cross-country unexplained differences in working hours might be driven by the dis(incentive) effects of benefits withdrawal when increasing hours of work, at least in some cases, such as the United Kingdom, Ireland, Switzerland and the Netherlands.

\subsubsection{Policies or preferences? (Still) an open debate?}

106. Country-fixed effects could also be interpreted as a measure of cultural differences, or, in the vein of Blanchard (2004), a measure of differences in preferences for leisure. Some authors have attempted investigating the strength of preferences through the use of public opinion surveys. One example is Cahuc et al. (2006) who compare public opinion surveys on the view of marriage and childcare across the Netherlands, Germany, France, Denmark, and Sweden, and find huge discrepancies in attitudes towards family life. Different sources of opinion surveys suggest that Dutch values are still much more geared towards women taking care of the family than in other European countries, notably the Scandinavian countries. ${ }^{73}$ The authors suggest that it is precisely these preferences, along with their evolution over time, that have favoured the emergence of institutional incentives promoting part-time work among women. This argument indicates the difficulty of disentangling the impact of policies from that of societal preferences, along with the potential interdependencies among the two. Going further, the idea that collective

\section{See Appendix.}

71. For an assessment of the so called "low-wage trap", see OECD (2005b, Chapter 3).

72. This variable is defined as the marginal effective tax rate for part-time employees for doubling of working hours from $1 / 3$ to $2 / 3$ of full-time hours. The figures represent averages over different family situations. The source of the variables is OECD Tax-Benefits Models.

73. For another piece of evidence on the Dutch experience, based on microeconomic data on female natives and immigrants' labour market behaviour in the Netherlands, see Bevelander and Groenveld (2007). 
preferences are embedded — or reflected — in regulations points to the impossibility of properly separate their respective roles in explaining cross-country differences.

Figure 6. Country fixed effects in the regression for women and marginal effective tax rates for part-time employees ${ }^{1}$

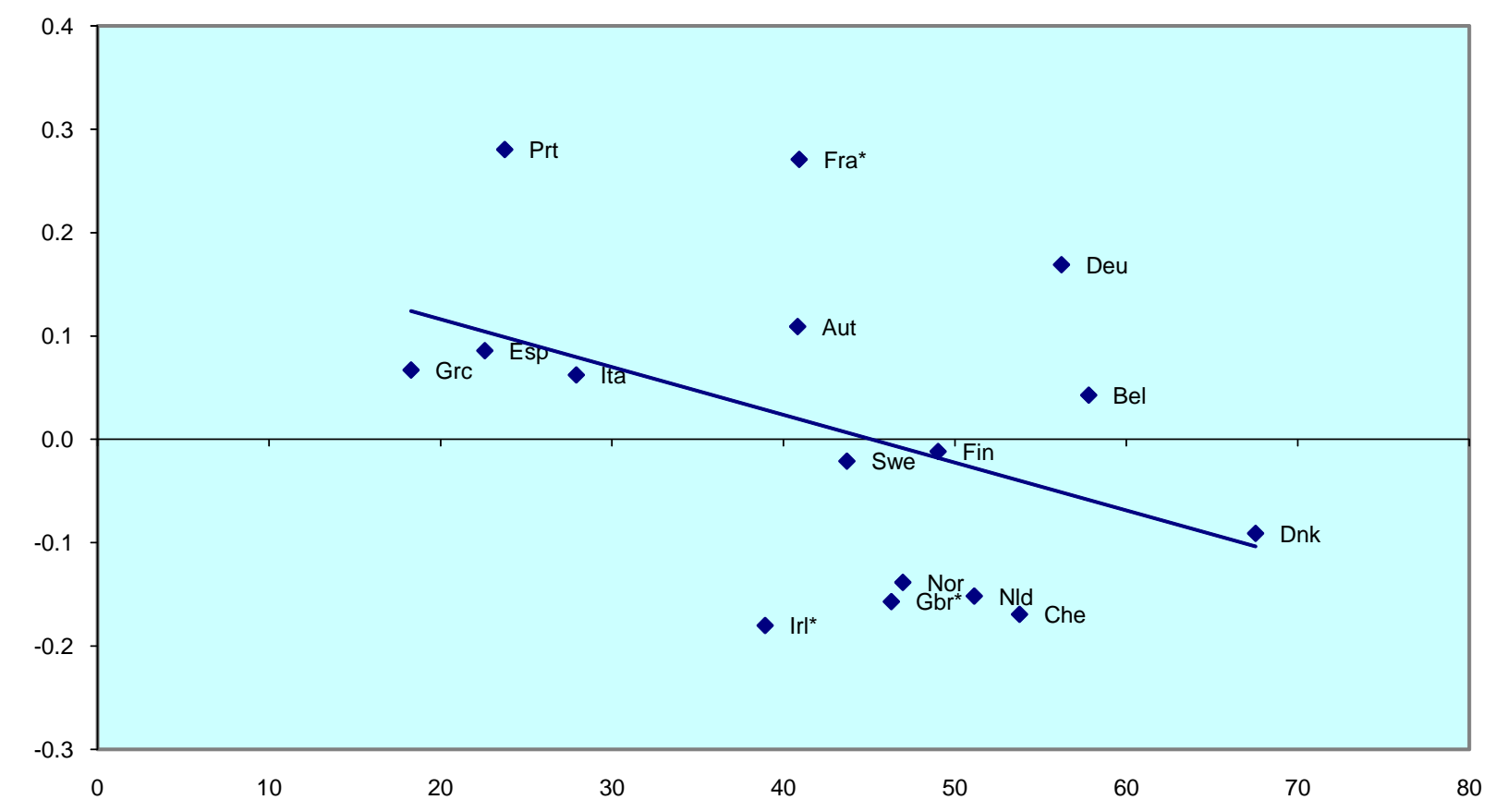

Notes: Statistical significance of the country fixed effects: ${ }^{*} p<.1,{ }^{\star \star} p<.05,{ }^{\star \star \star} p<.01$. Data refer to 2002.

1. Marginal effective tax rates for part time employees for doubling of working hours from $1 / 3$ to $2 / 3$ of full-time hours.

Average over different family situations (single with and without children, two family earners with and without children).

Source: OECD Tax-Benefits models.

6

Conclusion

107. This paper shows that policies and institutions do play a role in explaining differences in working hours among employed individuals across OECD countries. In particular, high marginal tax rates on second earners, most often married or cohabiting women, have a significant disincentive effect on the intensive margin of labour supply. Labour supply of men is found to be insensitive to taxation, as in earlier studies. Whereas working hours of women are responsive to taxation, working hours of men are found to be sensitive to working-time regulations, as well other product and labour market policies. Indeed, legislations regulating the length of working-time are found to be important for men, and in particular the low-skilled.

108. Employment protection legislation and anticompetitive product market policies are found to reduce hours worked by men, over and beyond their impact on employment rates. Unionisation has an opposite impact across genders, whereby it is associated with lower hours worked among men, and higher hours worked among women. These results are consistent with the idea that in highly regulated and unionised markets, insiders are able to negotiate shorter working hours, while outsiders, or marginal 
workers, exhibiting lower labour market attachment, are more sensitive to the financial (dis)incentives embedded in taxation systems. As documented in the paper, these findings can also be interpreted in terms of a labour demand framework.

109. While uncovering a role for policies and institutions in explaining differences in hours worked among OECD countries, the empirical work undertaken in this paper shows that an important proportion of the cross-country variation remains country specific, time invariant, and unexplained. Thus, the debate on "policies or preferences", remains to a large extent unresolved. As is suggested above, however, this controversy to a large extent hinges upon the false idea that it would be possible to disentangle the impact of institutional choices from that of societal preferences. Inter-dependencies among the two preclude this exercise to be undertaken.

110. This work indicates a number of important issues for future research. From a theoretical point of view, it suggests the need for a comprehensive framework reconciling microeconomic and macroeconomic models of labour supply. More work is also needed to model and estimate the inter-dependencies among intensive and extensive labour supply decisions, as well the adoption of a more general equilibrium framework, in which both labour supply and labour demand would be considered along the two margins. Finally, availability of time-use data on a cross-country and time-series basis would be of great value to study the impact of policies, and notably of taxation, along with the existence of social norms and eventually complementarities on the allocation of market work across family members. 
ECO/WKP(2008)4

\section{APPENDIX: COMPUTATION OF TAXATION VARIABLES}

111. This appendix describes the procedure for computing group specific taxation variables, based on the Taxing Wages models.

112. Marginal and average tax rates and tax wedges ${ }^{74}$ can be calculated for each per cent of gross earnings from $1 \%$ to $200 \%$ of the Average Production Worker wage (APW) along the following dimensions: ${ }^{75}$

- 30 countries: all OECD countries.

- 10 years: from 1996 to 2005.

- 6 family situation: single without children; ${ }^{76}$ single with 2 children; one-earner household without children; one-earner household with 2 children; two-earner household without children; two-earner household with 2 children.

- 2 earners: first and second earner (when appropriate).

113. This information needs to be "semi-aggregated" in order to be used in the statistical analysis, in particular to study the relationship with the disaggregated data of average hours worked. This is done by calculating simple averages of marginal tax wedges, marginal tax rates and average tax wedges along two or three dimensions, depending on the consideration of children in the household. In the baseline specification, due to data limitations on hours worked, only two dimensions can be exploited:

- Gender: men and women

- 2 family situations: single and married

For this aggregation, a number of assumptions are made:

- $\quad$ singles without children are both males and females

- $\quad$ singles with children are always females

- the only earner in the one-earner family is always male

- the first (second) earner in the two-earner family is always male (female)

74. Note that while marginal tax rates are defined at the individual level, average tax rates are defined at the household level.

75. The gross earnings range for the calculation is different in the case of the two-earner household. In this case, when the tax variables are calculated for the first earner, the second earner is assumed to earn $60 \%$ of APW. Thus, the gross earnings range for the first earner is $61 \%-200 \%$. When the tax variables are calculated for the second earner, the first earner is assumed to earn 100\% of APW. Thus the gross earnings range for the second earner is $1 \%-99 \%$ of APW.

76. The children are assumed to be aged between 5 and 12-years-old. 
114. The data allow to compute averages of tax indicators along a further dimension - the presence of children - although it is not possible, for an important number of countries and years, to match them with corresponding figures for hours worked in labour force surveys. For this reason, this analysis is undertaken as an extension for the limited number of countries covered by the data. In this case, the following dimensions are considered:

- Gender: men and women

- 4 family situation: single and married, with and without children

For this aggregation, a number of assumptions are made:

- singles without children are both males and females

- the only earner in the one-earner family is always male

- the first (second) earner in the two-earner family is always male (female)

115. It is assumed that gross earnings cannot be lower than $33 \%$ of APW. Indeed, the interaction of minimum wages and, potentially, working-time regulations makes it unlikely that labour income be below this threshold (see the special feature of the Taxing Wages devoted to taxation of minimum wages: OECD (2006), see also Annex A of Benefits and Wages, 2004 edition: OECD (2004a)).

116. Furthermore, note that in the Taxing Wages models, the standard definition of the average production worker for which the earnings figure is determined is assumed to be fully employed during the year. As shown in the special feature of the 2004/2005 Taxing Wages edition, the individuals earning 33\% of average earnings, who are considered in this work, are most likely to work part-time. However, the report shows that the assumption that all employees are working full time do not significantly affect the tax rates calculated in Taxing wages, except in the case of Belgium for married couples where the spouse is earning $33 \%$ of the average wage level. This is because in most OECD countries there are no hours-based tax provisions that are special for part-time workers.

\section{Limitations}

117. Another dimension on which the tax variables could be decomposed is skill level, proxied by the educational level. This would allow taxation variables used as regressors in the econometric analysis to vary according to the education level. There are two problems arising in this case:

1. This methodology might increase the endogeneity of tax rates with respect to hours worked in each category of workers.

2. This methodology increases measurement error in the construction of semi-aggregated taxation variables, by adding one additional and important assumption in the "mapping" rules running from Taxing Wages to LFS data.

118. A combination of tax increases and benefit withdrawals can reduce the financial incentives for increasing working hours or work effort. Marginal effective tax rates (METR) can be used to measure these disincentives. For low income groups, METR are useful indicators of so called "low-wage traps". Benefits and Wages, 2004 (OECD, 2004a) edition presents data on marginal effective tax rates for parttime employees (part-time being defined in proportion of the APW). The use of these data for low-income workers, proxied by the education level, would be interesting. However, these data are available for a very short period, precluding panel data techniques to be used consistently. Besides, it is arguable that accounting for benefit withdrawals is more important when studying the participation decision. 
119. Note that the impact of in-work benefits conditional on working a minimum of hours is imbedded in the empirical work done here to the extent that those benefits take the form of tax credits; this is the case of the most relevant "hours dependent" benefits programmes (such as the Working Families Tax Credit in the United Kingdom). ${ }^{77}$ Besides, the indicators considered here also take into account tax relieves and family cash transfers universally paid in respect of dependent children between 5 and 12 years of age who are attending school. (see Taxing Wages, OECD, 2005a).

77. See Benefits and Wages 2004 edition (OECD, 2004a), for an exhaustive review of the employmentconditional benefits programmes in OECD countries and the country-specific notes of Taxing Wages (OECD, 2006) for a description of the tax allowances and tax credits imbedded in the taxation indicators. 


\section{REFERENCES}

Aaberge, R., J.K. Dagsvik and S. Strøm (1990), "Labor Supply, Income Distribution and Excess Burden of Personal Income Taxation in Sweden", Report 22, Economic Research Programme on Taxation, Oslo.

Aaberge, R., J.K. Dagsvik and S. Strøm (1995), "Labor Supply Responses and Welfare Effects of Tax Reforms", Scandinavian Journal of Economics, Vol. 97, pp.635-659.

Aaberge, R., J.K. Dagsvik and S. Strøm (1999), "Labor Supply in Italy: An Empirical Analysis of Joint Household Decisions with Taxes and Quantity Constraints", Journal of Applied Econometrics, Vol. 14, pp.403-422.

Afsa, C. and P. Biscourp (2004), "L'évolution des rythmes de travail entre 1995 et 2001: quel impact des 35 heures ?", Economie et Statistique, Numéro 376-377.

Alesina, A., E. Glaeser and B. Sacerdote (2005), "Work and Leisure in the US and Europe: Why So Different?", NBER Working Paper No. 11278.

Altonji, J. and J. Oldham (2003), "Vacation Laws and Annual Work Hours." Economic Perspectives, Vol. 28, pp.19-29.

Amable, B., L. Demmou and D. Gatti (2006),"Institutions, Unemployment and Inactivity in the OECD Countries", PSE Working Papers No. 2006-16.

Arellano, M. and C. Meghir (1992), "Female Labour Supply and On the Job search: An Empirical Model Estimated Using Complementary Data Sets”, Review of Economic Studies, Vol. 59, pp.537-559.

Arrufat, J. and A. Zabalza (1986), "Female Labor Supply with Taxation, Random Preferences, and Optimization Errors", Econometrica, Vol. 54, pp.47-64.

Attanasio, O. and T. MaCurdy (1997), "Interactions in Family Labour Supply and their Implications for the Impact of EITC", Stanford University, mimeo.

Bassanini, A. and R. Duval (2006), "Employment Patterns in OECD Countries: Reassessing the Role of Policies and Institutions", Economics Department Working Papers No. 486, OECD, Paris.

Bargain, O. (2005), “On Modelling Household Labour Supply with Taxation”, IZA Working Paper No. 1455.

Barro, R. and C. Sahasakul (1983), "Measuring the Average Marginal Tax Rate from the Individual Income Tax", The Journal of Business, Vol. 56, pp.419-452.

Bell, L. A. and R. Freeman (2001), "The Incentive for Working Hard: Explaining Hours Differences in the US and Germany", Labor Economics, Vol. 8, pp. 181-202. 
Bertola, G., F.D. Blau, and L.M. Kahn (2002), "Labor Market Institutions and Demographic Employment Patterns", NBER Working Paper No. 9043.

Bevelander, P. and S. Groenveld (2007), "How Many Hours do you Have to Work to be Integrated? FullTime and Part-Time Employment of Native and Ethnic Minority Women in the Netherlands", IZA Discussion Paper No. 2684.

Blanchard, O. (2004), “The Economic Future of Europe”, Journal of Economic Perspectives, Vol. 18, pp.3-26.

Blau, F. and L. Kahn (2005), "Changes in the Labour Supply of Married Women: 1980-2000”, NBER Working Paper No, 11230.

Blomquist, S. (1983), “The Effect of Income Taxation on the Labor Supply of Married Men in Sweden”, Journal of Public Economics, Vol. 22, pp.169-197.

Blomquist, S. and U. Hansson-Brusewitz (1990), “The Effect of Taxes on Male and Female Labor Supply in Sweden", Journal of Human Resources, Vol. 25, pp.317-357.

Blundell, R. and I. Walker (1986), "Modelling the Joint Determination of Household Labour Supplies and Commodity Demands", Economic Journal, Vol. 92, pp.58-74.

Blundell, R., A. Duncan, and C. Meghir (1992), “Taxation and Empirical Labour Supply Models: Lone Parents in the UK", Economic Journal, Vol. 97, pp.44-64.

Blundell, R., A. Duncan, and C. Meghir (1998)," Estimating Labor Supply Responses Using Tax Reforms", Econometrica, Vol. 66, pp.827-861.

Blundell, R. and T. MaCurdy (1999), "Labor Supply: A Review of Alternative Approaches" in O. Ashenfelter and R. Layard (eds.), Handbook of Labor Economics, Vol. 3A, Amsterdam, North Holland.

Booth, A.L. and J.C. van Ours (2007), "Job Satisfaction and Family Happiness: the Part-Time Work Puzzle”, IZA Discussion paper No. 3020.

Bourguignon, F. and T. Magnac (1990), "Labor Supply and Taxation in France”, Journal of Human Resources, Vol. 25, pp.358-389.

Bowles, S. and Y. Park (2005), "Emulation, Inequality, and Work Hours: Was Thorsten Veblen Right?", Economic Journal, Vol. 115, pp.397-410.

Brewern, M. and J. Browne (2006), "The Effect of the Working Families Tax Credit on Labor Market Participation”, IFS BriefingNnotes, No. 69.

Bryan, M.L. (2006), "Free to Choose? Differences in the Hours Determination of Constrained and Unconstrained Workers", Oxford Economic Papers, Vol. 59, pp.226-252.

Buddelmeyer, H., G. Mourre, and M. Ward (2004), “The Determinants of Part-Time Work in EU Countries; Empirical Investigations with Macro Panel Data”, IZA Discussion Paper No. 1361.

Burda, M., D. Hamermesh, and P. Weil (2006), "The Distribution of Total Work in the EU and the US", IZA Discussion Paper No. 2270. 
Burda, M., D. Hamermesh and P. Weil (2007), "Total Work, Gender, and Social Norms", NBER Working Paper No. 13000.

Burgoon, B. and P. Baxandall (2004), "Three Worlds of Working-Time: Policy and Politics in Work-Time Patterns of Industrialized Countries", Paper prepared for presentation at the American Political Science Association Annual meeting, Boston, MA, 28 August-1 September, 2002.

Calmforst, L. and J. Driffil (1988), "Bargaining Structure, Corporatism and Macroeconomic Performance", Economic Policy, No. 6, April.

Calmfors, L. and M. Hoel (1988), "Work Sharing and Overtime", Scandinavian Journal of Economics, Vol. 90, pp.45-62.

Cahuc, P., B. Crépon, F. Kramarz, G. van Lomwell, T. Schank, O.N. Skans, and A. Zylberberg (2006), "Labor Market Effects of Work Sharing Arrangements in Europe", paper presented at the conference "Are Europeans Lazy? or Americans Crazy?" at the Fondazione Rodolfo Benedetti, Portovenere, Italy.

Cahuc, P. and A. Zylberberg (2003), Microéconomie du Marché du Travail, collection Repères, édition La Découverte.

Causa, O, and S. Jean (2007), "Integration of Immigrants in OECD Markets: Do Policies Matter?", OECD Economics Department Working Papers No. 564.

Chiappori, P.A. (1992), “Collective Labor Supply and Welfare”, Journal of Political Economy, Vol. 100, pp.437-467.

Chang, Y. and S. B. Kim (2005), "On the Aggregate Labor Supply", Federal Reserve Bank of Richmond Economic Quarterly, Volume 91/1, pp. 21-37.

Colombino, U. and D. del Boca (1990), The Effect of Taxes on Labor Supply in Italy, Journal of Human Resources, Vol. 25, pp.390-414.

Conway, P., D. De Rosa, G. Nicoletti and F. Steiner (2006), "Regulation, Competition, and Productivity Convergence", OECD Economics Department Working Papers No. 509.

Crépon, B. and F. Kramarz (2002), "Employed 40 Hours or Not Employed 39: Lessons from the 1981 Mandatory Reduction of the Weekly Working Hours", Journal of Political Economy, Vol. 110, pp.1355-1389.

Daveri, F. and G. Tabellini (2000), “Unemployment and Taxes”, Economic Policy, April, pp. 49-104.

Davis, S.J. and M. Henrekson (2004), “Tax Effects on Work Activity, Industry Mix and Shadow Economy Size: Evidence from Rich-Country Comparisons", NBER Working Paper No. 1056.

Devereux, P.J. (2004), "Changes in Relative Wages and Family Labor Supply”, The Journal of Human Resources, Vol. 39, pp.694-722.

Dew-Becker, I. and R.J. Gordon (2006), "The Slowdown in European Productivity Growth: A Tale of Tigers, Tortoises, and Textbook Labor Economics", paper presented at NBER Summer Institute Macroeconomics and Productivity Workshop, Cambridge, MA, July 20, 2006. 
Del Boca, D. (2002), "The Effect of Child Care and Part-Time Opportunities on Participation and Fertility Decisions in Italy", Journal of Population Economics, Vol. 15, pp.54-573.

Eissa, N. and S. Giertz (2006), "Trends in High Incomes and Behavioral Responses to Taxation: Evidence from Executive Compensation and Statistics of Income Data", Congressional Budget Office Working Papers, No. 2006-14

Evers, M., R.A. de Mooij and D.J. van Vuuren (2006), "What Explains the Variation in Estimates of Labour Supply Elasticities?", Tinbergen Institute Discussion Paper 2006-017/3.

Faggio, G. and S. Nickell (2007), "Patterns of Work Across the OECD”, The Economic Journal, Vol. 117, pp.416-F440.

Fang, L. and R. Rogerson (2007), "Policy Analysis in a Matching Model with Intensive and Extensive Margins", NBER Working Paper No.13007.

Fiori, G., G. Nicoletti, S. Scarpetta, and F. Schiantarelli (2007), "Employment Outcomes and the Interaction between Product and Labour Market Deregulation: Are They Substitutes or Complements?", IZA Discussion Paper No. 2770.

Fondazione Rodolfo de Benedetti (2006), Conference on "Are Europeans Lazy? or Americans Crazy?", Portovenere, Italy.

Geiner, J. and V. Steiner (2007), "Short-Run and Long-Term Effects of Childbirth on Mothers' Employment and Working Hours Across Institutional Regimes: An Empirical Analysis Based on the European Community Household Panel", IZA Discussion Paper No. 2693.

Griffith, R., R. Harrison and G. Macartney (2006), "Product Market Reforms, Labour Market Institutions and Unemployment," IFS Working Paper No. WP06/06.

Gronau, R. (1986), "Home Production: A Survey," in O. Ashenfelter and R. Layard (eds.) Handbook of Labor Economics, pp.273-304. Elsevier Science Publishing, Amsterdam.

Haan, P. and M. Myck (2006), “Apply with Caution: Introducing UK-Style In-Work Support in Germany”, DIW Discussion Paper No.538, DIW, Berlin.

Hamermesh, D.S. (1993), Labour Demand, Princeton, Princeton University Press.

Hausman, J. (1981), "The Effect of Taxes on Labour Supply”. In: H. Aaron and J. Pechman (eds.), How Taxes Affect Economic Behavior, Brookings, Washington D.C.

Heckman, J. (1976), “The Common Structure of Statistical Model of Truncation, Sample Selection, and Limited Dependent Variables and a Simple Estimator for Such Models", Annals of Economic and Social Measurement, Vol. 5, pp.475-492.

Heckman, J. (1979), "Sample Selection Bias as a Specification Error: An Application to the Estimation of Female Labor Supply Functions", in James Smith (ed.), Female Labor Supply, Princeton, NJ: Princeton University Press, 1980, pp.206-248.

Heckman, J. (1983), "Lessons from Empirical Labor Economics: 1972-1992”, American Economic Review, Paper and proceedings of the American Economic Association, Vol. 83, pp.116-12. 


\section{ECO/WKP(2008)4}

Hodrick, R.J. and E.C. Prescott, (1997), "Postwar US Business Cycles: An Empirical Investigation”, Journal of Money, Credit and Banking, Vol. 29, pp.1-16.

Houseman, S. (2001), "Why Employers Use Flexible Staffing Arrangements: Evidence from an Establishment Survey", Industrial and Labor Relations Review, Vol. 55, pp.149-169.

Huberman, M. and C. Minns (2005), "Hours of Work in Old and New World: The Long View, 18702000", IIIS Discussion Paper No. 95.

Hunt, J. (1999), “Has Work Sharing Worked in Germany?”, Quarterly Journal of Economics, Vol. 114, pp.117-148.

Immervol, H. and S. Barber (2005), "Can Parents Afford Work? Childcare Costs, Tax Benefit Policies, and Work Incentives", OECD ELS Working Paper No. 31.

Immervol, H., J. Kleven, C.T. Kreiner and E. Saez (2007), "Welfare Reform in European countries: A Microsimulation Analysis to Assess Alternative Scenarios", Economic Journal, Vol. 117, pp.1-44.

Jimeno, J.J. and D. Rodriguez-Palenzuela (2002), "Youth Unemployment in the OECD: Demographic Shifts, Labor Market Institutions, and Macroeconomic Shocks", European Central Bank Working Paper No. 155.

Jaumotte, F. (2003), "Female Labour Force Participation: Past Trends and Main Determinants in OECD Countries”, OECD Economics Department Working Papers No. 376, OECD, Paris.

Kaiser, H., U. van Essen and P.B. Spahn (1992), "Income Taxation and the Supply of Labour in West Germany”, Jahrbucher für Nationaokonomie und Statistik, Vol. 209, pp.87-105.

Kooreman, O. and A. Kapteyn (1986), "Estimation of Rationed and Unrationed Household Labor Supply Functions Using Flexible Functional Forms", Economic Journal, Vol. 96, pp.308-322.

Kuismanen, M. (1997), "Labour Supply, Unemployment and Income Taxation: An Empirical Application”, Working Paper, Government Institute for Economic Research, Helsinki, University College London.

Kuroda, S. and I. Yamamoto (2007) "Estimating Frisch Labour Supply Elasticity in Japan”, Discussion Paper No. 2007- E-5, Institute for economic and monetary studies, Bank of Japan.

MaCurdy, T., P. Green and H. Paarsch (1990), "Assessing Empirical Approaches for Analyzing Taxes and Labor Supply”, Journal of Human Resources, Vol. 25, pp.415-490.

Michelacci, C. and J. Pijoan-Mas (2007), "The Effects of Labour Market Conditions on Working Time: The US EU Experience", CEPR Discussion Paper No. 6314.

Moffitt, R. and M. Wilhelm (2000), "Taxation and the Labor Supply Decisions of the Affluent." in J. Slemrod (ed.), Does Atlas Shrug? The Economic Consequences of Taxing the Rich, Cambridge University Press.

Mroz, T.A. (1987), “The Sensitivity of an Empirical Model of Married Women's Hours of Work to Economic and Statistical Assumptions", Econometrica, Vol. 55, pp. 765-799. 
Nickell, S. and R. Layard (1999), "Labor Market Institutions and Economic Performance", in O. Ashenfelter and D. Card (eds.) Handbook of Labor Economics. Vol. 3C, North-Holland, Amsterdam.

Nicoletti, G. and S. Scarpetta (2005), "Product Market Reforms and Employment in OECD Countries", OECD Economics Department Working Papers, No. 472, OECD, Paris.

Nunziata, L. (2005), "Institutions and Wage Determination: A Multi Country Approach", Oxford Bulletin of Economics and Statistics, Vol. 67, pp.435-465.

OECD (2004a), Benefits and wages - OECD Indicators, Paris.

OECD (2004b), Babies and Bosses: Reconciling Work and Family Life, Paris.

OECD (2005a), Taxing Wages 2004-2005 Paris.

OECD (2005b), Employment Outlook, Paris.

OECD (2006), Taxing Wages 2005-2006, Paris.

OECD (2007), Economic Policy reforms: Going for Growth, Paris.

Ohanian, L., A. Raffo, and R. Rogerson (2006), "Long-Term Changes in Labor Supply and Taxes: Evidence from OECD Countries, 1956-2004”, NBER Working Paper No. 1276.

Pencavel, J. (1986), "Labor Supply of Men: A Survey”, in O. Ashenfelter and R. Layard (eds.), Handbook of Labor Economics, Vol. 1, Amsterdam, North Holland, pp.3-102.

Prescott, E. (2004), “Why Do Americans Work So Much More Than Europeans?”, Federal Reserve Bank of Minneapolis Quarterly Review, Vol. 28, pp.2-13.

Rammohan, A. and S. Whelan (2007), "The Impact of Childcare Costs on the Full-Time/Part-Time Employment Decisions of Australian Mothers", Australian Economic Papers, Vol. 46, pp.152-169.

Raven, O. and H. Uhlig (2002), "On Adjusting the Hodrick-Prescott Filter for the Frequency of Observations", Review of Economics and Statistics, Vol. 84, pp.371-380.

Ransom, M. R. (1987), "The Labor Supply of Married Men: A Switching Regressions Model", Journal of Labour Economics, Vol. 5, pp. 63-75.

Rogerson, R. (2005), "Understanding Differences in Hours Worked", Review of Economic Dynamics, Vol. 9, pp.365-409.

Rogerson, R. (2006), “Taxation and Market Work: Is Scandinavia an Outlier?”, Arizona State University.

Rogerson, R. and J. Wallenius (2006), "Micro and Macro Elasticities in a Life Cycle Model with Taxes", NBER Working Paper No. 13017.

Rosen, S. (1978), "The Supply of Work Schedules and Employment." in Work Time and Employment, National Commission on Manpower Policy, pp.145-173. 


\section{ECO/WKP(2008)4}

Rose-Birch, E. (2005), "Studies of the Labour Supply of Australian Women: What have we Learned?", Economic Record, Vol. 81, pp.65-84.

Saez, E (2003), "The Effect of Marginal Tax Rates on Income: A Panel Study of 'Bracket Creep", Journal of Public Economics, Vol. 87, pp.1231-1258.

Skuterud, M. (2007), "Identifying the Potential of Work Sharing as a Job Creation Strategy", Journal of Labor Economics, Vol. 25, pp.265-287.

Smith, J.B. and M. Stelcner (1988), "Labour Supply of Married Women in Canada, 1980”, Canadian Journal of Economics, Vol. 21, pp.857-870.

Stephenson, E.F. (1998), “Average Marginal Tax Rates Revisited”, Journal of Monetary Economics, Vol. 41, pp.389-409.

Triest, R. (1990), “The Effect of Income Taxation on Labor Supply in the United States”, Journal of Human Resources, Vol. 25, pp.491-516

Van Soest, A., I. Woittiez and A. Kapteyn (1990), "Labor Supply, Income Taxes, and Hours Restrictions in the Netherlands", Journal of Human Resources, Vol. 25, pp.517-558. 


\section{WORKING PAPERS}

The full series of Economics Department Working Papers can be consulted at www.oecd.org/eco/Working_Papers/

595 Fiscal policy in India: past reforms and future challenges (February 2008) Richard Herd and Willi Leibfritz

594. The significance of Switzerland's enormous current-account surplus (March 2008) Peter Jarrett and Céline Letremy

593. Interdependencies between monetary policy and foreign-exchange intervention under inflation targeting: the case of Brazil and the Czech Republic

(Janvier 2008) Jean-Yves Gnabo, Luiz de Mello and Diego Moccero

592 Solow or Lucas? Testing growth models using panel data from OECD countries (December 2007) Jens Arnold, Andrea Bassanini and Stefano Scarpetta

591 The private internal rates of return to tertiary education: new estimates for 21 OECD countries (December 2007) Romina Boarini/Hubert Strauss.

590 Making federalism work

(December 2007) Alexandra Bibbee

589 The Wage premium on tertiary education: New Estimates for 21 OECD countries (December 2007) Hubert Strauss/Christine de la Maisonneuve

588 Enhancing the benefits of financial liberalisation (March 2007) Stefan Ide, Jens Høj, Patrick Lenain

587. Improving Incentives in Tertiary education (Marcch 2007) Jens Høj

586 Globalisation and the European Union: which countries are best placed to cope? (December 2007) David Rae, Marte Sollie

585 Primary and Secondary education in the United States (November 2007) Peter Tulip and Gregory Wurzburg

584 Financing higher education in the United States (November 2007) Peter Tulip

583 Corporate Net Lending: a review of recent trends (November 2007) Christophe André, Stéphanie Guichard, Mike Kennedy and David Turner

581. Local government finances: The link between intergovernmental transfers and net worth (September 2007) Luiz de Mello

580. Boosting Austria's Innovation Performance Improving Innovation (September 2007) Willi Leibfritz and Jürgen Janger 


\section{ECO/WKP(2008)4}

579. Improving Employment Prospects in the Slovak Republic: Building on Past Reforms (September 2007) Andres Fuentes

578. Improving education outcomes in the Slovak Republic (September 2007) David Carey

577. Regulatory reforms in Sweden have boosted productivity (September 2007) Espen Erlandsen and Jens Lundsgaard

576. The policy determinants of investment in tertiary education (September 2007) Joaquim Oliveira Martins, Romina Boarini, Hubert Strauss, Christine de la Maisonneuve and Clarice Saadi

575. Product market competition in the OECD countries: taking stock and moving forward (September 2007) Jens Høj, Miguel Jimenez, Maria Maher, Giuseppe Nicoletti, and Michael Wise

574. Too little destruction too little creation: A Schumpeterian diagnosis of barriers to sustained growth in Ukraine

(September 2007) Christian Gianella and William Tompson

573. How do the OECD Growth Projections for the G7 Economies Perform? A post-mortem. (September 2007) Lukas Vogel

572. Austria's deepening economic integration with Central and Eastern Europe (August 2007) Rina Bhattacharya

571. Meeting the challenges of decentralization in France (July 2007) Stéphanie Jamet

Faire face aux défis de la décentralisation en France (juillet 2007) Stéphanie Jamet

570. Enhancing incentives to improve performance in the education system in France (July 2007) Paul O'Brien

Renforcer les incitations à une meilleure performance du système éducatif en France (juillet 2007) Paul O’Brien

569. Combating poverty and social exclusion in France

(July 2007) Stéphanie Jamet

Lutter contre la pauvreté et l'exclusion social en France

(juillet 2007) Stéphanie Jamet

568 The competition law and policy indicator (July 2007) Jens Hoj

567. Structural policies and economic resilience to shocks (July 2007) Romain Duval, Jørgen Elmeskov and Lukas Vogel

566. Family policy in Hungary: how to improve the reconciliation between work and family? (July 2007) Philip Hemmings 